\title{
Aspects of Integrable Models
}

\author{
UNDERGRADUATE THESIS
}

Submitted in partial fulfilment of the requirements of

BITS F421T Thesis

By

Pranav DIWAKAR

ID No. 2014B5TS0711P

Under the supervision of:

Dr. Sujay AsHoK

$\&$

Dr. Rishikesh VAIDYA

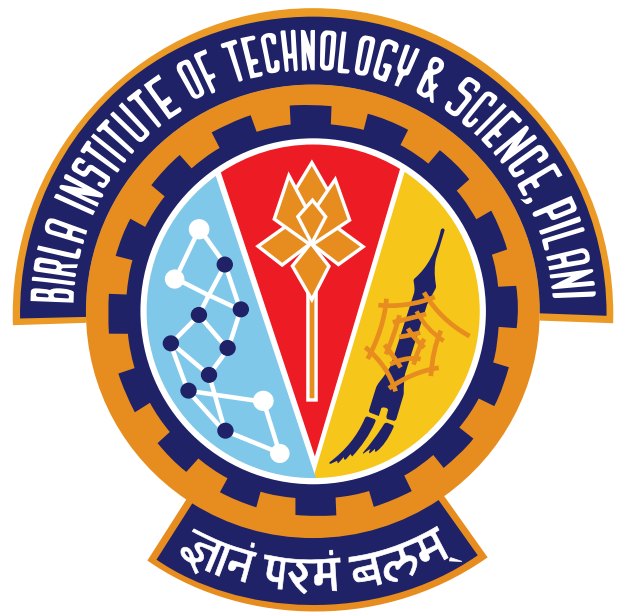

BIRLA INSTITUTE OF TECHNOLOGY AND SCIENCE PILANI, PILANI CAMPUS January 2018 


\section{Declaration of Authorship}

I, Pranav Diwakar, declare that this Undergraduate Thesis titled, 'Aspects of Integrable Models' and the work presented in it are my own. I confirm that:

- This work was done wholly or mainly while in candidature for a research degree at this University.

- Where any part of this thesis has previously been submitted for a degree or any other qualification at this University or any other institution, this has been clearly stated.

- Where I have consulted the published work of others, this is always clearly attributed.

- Where I have quoted from the work of others, the source is always given. With the exception of such quotations, this thesis is entirely my own work.

- I have acknowledged all main sources of help.

Signed:

Date: 


\section{Certificate}

This is to certify that the thesis entitled "Aspects of Integrable Models" and submitted by Pranav DIWAKAR, ID No. 2014B5TS0711P, in partial fulfillment of the requirements of BITS F421T Thesis embodies the work done by him under my supervision.

Supervisor

Dr. Sujay Ashok

Associate Professor,

The Institute of Mathematical Sciences

Date:
Co-Supervisor

Dr. Rishikesh VAIDYA

Assistant Professor, BITS Pilani, Pilani Campus

Date: 


\title{
BIRLA INSTITUTE OF TECHNOLOGY AND SCIENCE PILANI, PILANI CAMPUS
}

\author{
Abstract \\ Master of Science (Hons.)

\section{Aspects of Integrable Models}

by Pranav DiwaKar

The objective of this thesis is to study the isotropic XXX-1/2 spin chain model using the Algebraic Bethe Ansatz. To this end, we discuss the concept of integrability as well as the Lax operator and R-matrix, which help generate as many commuting operators in involution as there are degrees of freedom. We establish that the spin chain Hamiltonian belongs to this set and provide a definition of a state vector whose parameters, the Bethe roots, are constrained by a set of equations called the Bethe Ansatz Equations. We show that there is a one-to-one correspondence between the Bethe roots and the eigenfunctions of the system. Next, we proceed to study the nature of the low-lying excitations of both the ferromagnetic and antiferromagnetic model in the thermodynamic limit $N \rightarrow \infty$ and show that the Bethe roots can be grouped into complexes or strings, which behave like bound states. We see that integrability is directly related to diffractionless scattering, which is obeyed by systems whose scattering matrices satisfy the Yang-Baxter Equation. In order to provide a more physical interpretation, we calculate the scattering matrix of the two-body problem for a system that satisfies the Yang-Baxter Equation and obtain exchange relations that are identical to those obtained using the Algebraic Bethe Ansatz for the XXX-1/2 spin chain model. Finally, we calculate the scattering matrix for a two-body problem interacting with a delta potential and show that this is the same as what we derived using the Coordinate Bethe Ansatz. 


\section{Acknowledgements}

This thesis would be far from complete if not for the patient guidance of my supervisor, Dr Sujay Ashok, who I am greatly indebted to for helping me to get a foot in the door of theoretical physics, by directing me on every step I took from understanding the physics behind the equations to working through the mathematics that is embodied by the equations below. This whole work and my newfound fascination with the study of integrable systems were realised through innumerable discussions with him, and I am most grateful.

I would also like to thank my co-supervisor, Dr Rishikesh Vaidya, who has been a pillar of support since the beginning of my degree and nurtured my interest in physics through multiple courses and discussions throughout the last few years.

I would like to express my heartfelt gratitude to Dr Ganapathy Baskaran, Dr Balachandran Sathiapalan, and Dr R. Ganesh from the Institute of Mathematical Sciences for constantly helping me put my interests in perspective since my second year. While I didn't work directly with them, it is unlikely that I would have been able to perform this thesis in the Institute if not for their assistance.

I am most grateful to the governing board, faculty, and staff of The Institute of Mathematical Sciences for allowing me to make use of their facilities without objection and for providing me with an organic environment to carry out my work for the last five months.

I would like to thank the Vice Chancellor and Director of BITS, Pilani as well as the Head, faculty, and staff of the Department of Physics, BITS Pilani, for allowing me to complete my thesis outside the Institute and for providing me with the foundation that allowed me to approach such an advanced area of research. 


\section{Contents}

Declaration of Authorship $\quad$ i

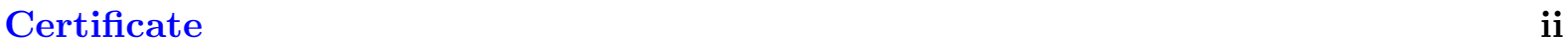

Abstract $\quad$ iii

Acknowledgements $\quad$ iv

Contents $v$

1 Introduction 1

2 Integrability 3

2.1 Classical Integrability $\ldots \ldots \ldots \ldots \ldots \ldots$

2.1 .1 The Lax operator . . . . . . . . . . . . . . . . 4

2.2 Quantum Integrability and Diffractionless Scattering . . . . . . . . . . . . 5

3 Analysis of the $\mathrm{XXX}_{1 / 2}$ Model 6

3.1 Coordinate Bethe Ansatz . . . . . . . . . . . . . . . . . . 6

3.2 Algebraic Bethe Ansatz . . . . . . . . . . . . . . . . . . . . . 10

3.2.1 Fundamental Commutation Relations . . . . . . . . . . . . . . . . 11

3.2.2 Monodromy and the Family of the Transfer Matrix . . . . . . . . . . . . . . 13

3.2.3 Deriving the Bethe Ansatz Equations . . . . . . . . . . . . . . . 18

3.2.4 $\mathrm{XXX}_{1 / 2}$ model in the ferromagnetic thermodynamic limit $(N \rightarrow \infty) \ldots 25$

3.2 .5 BAE for an arbitrary configuration . . . . . . . . . . . . 28

3.2.6 Dimensionality of the Spin Chain with the Complex Hypothesis . . . . . . 32

3.2.7 $\mathrm{XXX}_{1 / 2}$ model in the antiferromagnetic thermodynamic limit . . . . . . . 34

4 Scattering and Interaction of Magnons $\quad 43$

4.1 Yang-Baxter Equations through Consistency Conditions . . . . . . . . . . . . . 43

4.2 The Two-body Problem with a Delta Potential . . . . . . . . . . . . . . . 51

5 Summary and Conclusion $\quad \mathbf{5 5}$

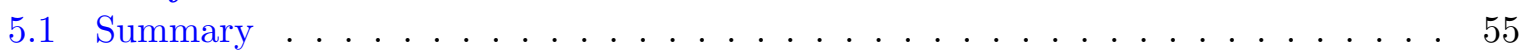

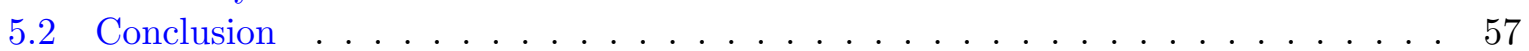


A Proofs of Select Equations from the Analysis of $\mathrm{XXX}_{1 / 2}$ Model $\mathbf{5 8}$

A.1 Proof of RLL Relation $(3.28) \ldots \ldots \ldots \ldots \ldots$

A.2 Derivation of Exchange Relations $(3.56) \ldots \ldots \ldots \ldots \ldots$. . . . . . . . . . . . . . . . 61

A.3 Derivation of form of $M_{j}(\lambda, \lambda)(3.67) \ldots \ldots \ldots \ldots \ldots$

A.4 Eigenvalue of Bethe vectors when acted on by $S^{3}(3.80) \ldots \ldots \ldots \ldots . \ldots 6$

A.5 Momentum, Energy, and S-Matrix Element of a type-M Complex (3.104, 3.105,

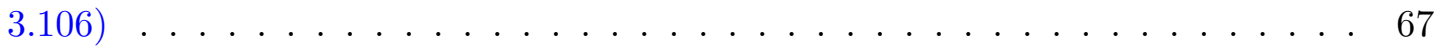

A.6 Results used in the Derivation of the Dimensionality of the Spin Chain (3.2.6) . . 70

A.7 Results Used in Analysis of Antiferromagnetic Ground State in Thermodynamic

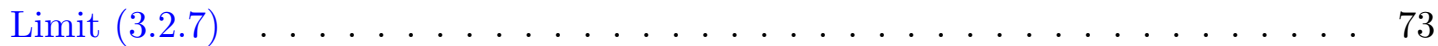


To the future. 


\section{Chapter 1}

\section{Introduction}

The study of integrable systems over the last fifty years has cemented the subject's place in a wide range of physical topics that are becoming increasingly relevant today. Starting with the formulation of the Korteweg-de Vries equation by Bossinesq (1877), later revisited by Korteweg and de Vries themselves (1895), the study of these exactly solvable models has grown to become a field of its own.

The concept of an integrable system is closely related to Noether's theorem and conservation laws. By definition, a classical system is integrable if it has as many independent constants of motion as it does degrees of freedom, with these constants being in involution. In its quantum form, this means that the integrability of a system is dependent on whether these constants of motion, which become operators in the quantum treatment, commute with the Hamiltonian and with each other. Their commutation implies that these operators share a common set of eigenstates with the Hamiltonian and therefore, finding the eigenstates for any of these operators is equivalent to finding the solutions of the Hamiltonian. In Chapter 2, an introduction to the idea of integrability and the Lax formulation is presented.

Historically, there have been numerous approaches to solving for the wavefunctions of integrable models. However, the one that has best stood the test of time is the Bethe Ansatz. First employed by Hans Bethe in his paper 'On the Theory of Metals' (1931), a coordinate ansatz, or guess, was made for the eigenfunctions of the Hamiltonian of the one-dimensional XXX-1/2 Heisenberg spin chain. While the first solution employed a coordinate Ansatz, L.D. Faddeev devised a new technique which required understanding the model with creation and annihilation operators acting on eigenstates, and subsequently finding the eigenvalues of the eigenstates when acted on by these operators. This technique has come to be known as the Algebraic Bethe Ansatz, or the Quantum Inverse Scattering Method. The algebraic and coordinate Bethe Ansatz techniques both yield the exact same results and constraints for the model, and the focus will be on the former in this thesis. Chapter 3 gives a brief insight into the Coordinate 
Bethe Ansatz and proceeds to describe the Algebraic Bethe Ansatz, by using the technique with the XXX-1/2 model to find the eigenfunctions. Select wavefunctions in both the ferromagnetic and the antiferromagnetic chains are studied and it is shown that the lowest-lying excitations correspond to bound states. It is also seen that the flipping of spins can correspond to the creation of quasiparticles.

Chapter 4 studies the implications that integrability has on the nature of two-body or many-body scattering. It is seen that scattering in integrable systems is diffractionless and the S-matrices corresponding to these obey a fundamental equation which allows for the eigenfunctions to be found. Following this, the Algebraic Bethe Ansatz is generalised to any integrable system. Finally, the potential of the spin chain model is investigated to complete the picture.

The Algebraic Bethe Ansatz was found to be a great tool in finding solutions of many other integrable systems, including the Sine-Gordon, Bose gas, and Non-linear Schrodinger models. Historically, the most striking part of the investigations of these models was that, for particular limits, they yielded the same forms of governing equations and operators. While this thesis only studies the isotropic XXX model, it arrives at the same results in multiple ways, with each method corresponding to a different physical treatment of the model. This correspondence highlights the accuracy of the Bethe Ansatz as a technique for solving integrable systems.

While the Bethe Ansatz started as a condensed matter problem, it has now branched out to include multiple fields of physics. The study of integrable systems has now become so vast as to include topics like many-body localisation and correspondences with supersymmetric gauge theory and superstrings are being examined in earnest. Hence, the study of the Bethe Ansatz will prove to be fruitful. 


\section{Chapter 2}

\section{Integrability}

\subsection{Classical Integrability}

Let the Hamiltonian of a system be $H\left(q_{i}, p_{i}\right)$, where $i=1,2 \ldots N . N$ is the number of degrees of freedom. The Hamilton equations of motion are

$$
\begin{gathered}
\dot{q}_{i}=\frac{\partial H}{\partial p_{i}} \\
\dot{p}_{i}=-\frac{\partial H}{\partial q_{i}}
\end{gathered}
$$

Classically, a system is said to be integrable if the number of degrees of freedom is equal to the number of constants of motion with respect to time and these constants are in involution.

That is, there exist $N$ constants of motion $\left\{F_{i}\right\}$ which satisfy

$$
\left\{F_{j}, F_{k}\right\}=0
$$

where $j, k=1,2 \ldots N$.

The Hamiltonian is time-independent and is, hence, one of these constants of motion. Therefore, $\left\{F_{i}, H\right\}=0$ for all $i=1,2 \ldots N$. Hence, if $N-1$ more constants of motion can be found, the system can be said to be integrable. Integrability, in its simplest sense, means that there exists an exact solution for the equations of motion and there is no chaotic behaviour. 


\subsubsection{The Lax operator}

While it is easy to verify that a set of constants of motion are in involution and the system is therefore integrable, the process of finding these constants is much more difficult. The constants of motion in most integrable systems have been found through trial-or-error by essentially making an ansatz or 'guess'. In order to simplify this, many previous studies have used the idea of a Lax pair.

The Lax pair is essentially a pair of matrices, say, $L$ and $M$, which satisfy the condition

$$
\frac{d L}{d t}=[M, L]
$$

The Lax pair is useful because it generates constants of motion. These can be found by the formulation

$$
F_{k}=\operatorname{tr} L^{k}
$$

This is justified because the quantities $\left\{F_{k}\right\}$ are conserved, as shown below using the property that the trace remains the same upon cyclic permutations.

$$
\begin{array}{r}
\frac{d}{d t} F_{k}=\frac{d}{d t} \operatorname{tr} L^{k}=\sum_{i=1}^{k} \operatorname{tr}\left(L^{i} \frac{d L}{d t} L^{k-(i+1)}\right)=\sum_{i=1}^{k} \operatorname{tr}\left(L^{k-(i+1)} L^{i} \frac{d L}{d t}\right)=\sum_{i=1}^{k} \operatorname{tr}\left(L^{k-1} \frac{d L}{d t}\right) \\
=\sum_{i=1}^{k} \operatorname{tr}\left(L^{k-1}[M, L]\right)=k \operatorname{tr}\left(L^{k-1}[M, L]\right)=k\left\{\operatorname{tr}\left(L^{k-1} M L\right)-\operatorname{tr}\left(L^{k} M\right)\right\} \\
=k\left\{\operatorname{tr}\left(L^{k} M\right)-\operatorname{tr}\left(L^{k} M\right)\right\}=0
\end{array}
$$

Hence, $\left\{F_{k}=\operatorname{tr} L^{k}\right\}$ are constants of motion. However, the system can only be integrable if these are in involution. Let the Lax operators act on a space $V \otimes V$ such that $L_{1}=L \otimes I$ and $L_{2}=I \otimes L_{2}$. If we can find a matrix $r_{12}$ acting on $V \otimes V$ such that

$$
\left\{L_{1}(\lambda), L_{2}(\mu)\right\}=\left[r_{12}(\lambda-\mu), L_{1}(\lambda)+L_{2}(\mu)\right]
$$

We see that the above relation ensures that the conserved quantities $\left\{F_{k}\right\}$ are in involution, as shown below. 


$$
\begin{gathered}
\left\{F_{j}(\lambda), F_{k}(\mu)\right\}=\left\{\operatorname{trL}^{j}(\lambda), \operatorname{tr} L^{k}(\mu)\right\}=j k \operatorname{tr}_{12}\left\{L_{1}^{j-1}(\lambda)\left\{L_{1}(\lambda), L_{2}(\mu)\right\} L_{2}^{k-1}(\mu)\right\} \\
=j k \operatorname{tr}_{12}\left\{L_{1}^{j-1}(\lambda)\left[r_{12}(\lambda-\mu), L_{1}(\lambda)+L_{2}(\mu)\right] L_{2}^{k-1}(\mu)\right\} \\
=j k \operatorname{tr}_{12}\left\{L_{2}^{k-1}(\mu) L_{1}^{j-1}(\lambda) r_{12}(\lambda-\mu)\left(L_{1}(\lambda)+L_{2}(\mu)\right)\right\}-j k \operatorname{tr}_{12}\left\{L_{2}^{k-1}(\mu) L_{1}^{j-1}(\lambda)\left(L_{1}(\lambda)+L_{2}(\mu)\right) r_{12}(\lambda-\mu)\right\} \\
=0 \quad(2.8)
\end{gathered}
$$

Clearly, the constants of motion are also in involution.

Therefore, the constants of motion can be generated by the Lax operator and involution can be ensured by defining an appropriate R-matrix. As a result, our problem of finding integrable systems is reduced to defining an appropriate Lax operator and R-matrix, from which constants of motion can be generated. However, this task is much harder than it seems and mostly works by trial-and-error. At the same time, however, we see that the relations between the Lax operator and R-matrix are universal and should hold for all integrable systems. We can argue that if we find an appropriate R-matrix which satisfies the consistency condition above, the system to which this corresponds is integrable. Hence, the most crucial step in solving integrable systems is to define the Lax operator. In the section on the Algebraic Bethe Ansatz, we will show how knowing the Lax operator allows us to find the eigenstates and constraints on a system.

\subsection{Quantum Integrability and Diffractionless Scattering}

When we speak about quantum integrability, the Poisson brackets are replaced with commutators but the fundamental definition is the same. Hence, a system can be said to be integrable if there exist $N$ independent operators that commute with the Hamiltonian and with each other.

As we noted in the previous section, the Hamiltonian is one of the $N$ commuting operators. We know that commuting operators share a common set of eigenstates. Hence, if we can find the eigenvectors of any one of the commuting operators, we have found the eigenstates of the system.

Another fundamental characteristic of quantum integrability is that an integrable system is directly related to diffractionless scattering. Diffractionless scattering implies that when two or more particles scatter with each other, their momenta are either retained or exchanged. As we will see in the discussion of particle scattering in Chapter 4, any system which scatters without diffraction can be solved using the Bethe Ansatz. But before we begin that discussion, it is shown how the Bethe Ansatz uses the Lax operator to generate $N$ mutually commuting operators for the spin chain model and solves for the eigenfunctions. 


\section{Chapter 3}

\section{Analysis of the $\mathrm{XXX}_{1 / 2}$ Model}

\subsection{Coordinate Bethe Ansatz}

Bethe was the first to provide a form for the eigenstates of the Heisenberg spin chain. In his paper 'On the Theory of Metals', Bethe made the guess (or ansatz) that the wavefunction is planar. This made it necessary for him to assume that the spins were at discrete sites on a periodic one-dimensional lattice, in order to ensure that the wavefunction had the same form at $x$ and $x+N$, where $N$ is the total number of spins. With a ferromagnetic chain with spin- $1 / 2$ particles, Bethe considered the reference state or 'ground state' to be all spins pointing in the same direction. By flipping first one spin, and then two, and so on, Bethe showed that the excited states could be treated as quasi-particles called magnons.

This subsection provides a brief description of the 'coordinate Bethe Ansatz' and future subsections will show how this picture is confirmed by the algebraic Bethe Ansatz. The expression given for the Hamiltonian by Heisenberg is the following.

$$
H=\sum_{n, \alpha} J^{\alpha} S_{n}^{\alpha} S_{n+1}^{\alpha}
$$

We consider the chain to be ferromagnetic and $J>0$, in which case the Hamiltonian becomes the negative of the above Hamiltonian.

We take the reference state to be all spins pointing up and label it by

$$
|0\rangle \equiv|00 \ldots 00\rangle=|\uparrow \uparrow \ldots \uparrow \uparrow\rangle
$$


Using the expression for the Hamiltonian given in equation (3.1), it is trivial to see that the energy of this state is zero, confirming that it is the ground state. That is,

$$
H|0\rangle=0
$$

We note that the action of $S_{n}^{-}$flips the up spin at the $n$th site.

If there are $l$ flipped spins, we notate the corresponding state by

$$
\left|n_{1}, n_{2} \ldots n_{l}\right\rangle=S_{n_{1}}^{-} S_{n_{2}}^{-} \ldots S_{n_{l}}^{-}|0\rangle
$$

where $1 \leq n_{1} \leq n_{2} \cdots \leq N$.

A general eigenstate is, hence, given by

$$
|\psi\rangle=\sum a\left(n_{1}, n_{2} \ldots n_{l}\right)\left|n_{1}, n_{2} \ldots n_{l}\right\rangle
$$

The Bethe Ansatz is essentially postulating the form of the coefficients $a\left(n_{1}, n_{2} \ldots n_{l}\right.$. Bethe guessed the form of these to be akin to those of a plane wavefunction.

The state with one flipped spin at site $n$ is given by

$$
|n\rangle=S_{n}^{-}|0\rangle=\left|\ldots \downarrow_{n} \ldots\right\rangle
$$

which is the next logical state to consider. Since the spin chain is periodic, it doesn't matter which spin is flipped, giving $N$ equivalent states.

When this state is acted upon by the Hamiltonian, we get

$$
H|n\rangle=H\left|\ldots \downarrow_{n} \ldots\right\rangle=-\frac{J}{2}\left|\ldots \downarrow_{n-1} \ldots\right\rangle-\frac{J}{2}\left|\ldots \downarrow_{n+1}\right\rangle+J\left|\ldots \downarrow_{n} \ldots\right\rangle
$$

We note that the down spin can either stay in its initial position $n$ or it can move to either of its neighbouring sites $n-1$ and $n+1$. In a sense, the down spin behaves like a particle which can move through the ring. We call this quasiparticle a magnon.

Assuming that the magnon has a momentum $p$ and using the plane wave ansatz, we can write the eigenvector as 


$$
|p\rangle=\sum_{n=1}^{N} e^{i p n} S_{n}^{-}|0\rangle
$$

The quantisation condition $a(n+N)=a(n)$ is, hence, equivalent to $e^{i p N}=1$. This clearly quantises the momentum and if we can obtain the dispersion relation, will quantise the energy as well.

Acting the Hamiltonian on this state, we have

$H|p\rangle=\sum_{n=1}^{N}-J\left[\frac{1}{2}\left(e^{i p(n-1)}+e^{i p(n+1)}\right)-e^{i p n}\right]\left|\ldots \downarrow_{n} \ldots\right\rangle=J(1-\cos p) \sum_{n=1}^{N} e^{i p n}\left|\ldots \downarrow_{n} \ldots\right\rangle=2 J \sin ^{2} \frac{p}{2}|p\rangle$

Hence, by the analogy of this excitation being a quasiparticle, its energy is

$$
E=2 J \sin ^{2} \frac{p}{2}
$$

which is positive. Hence, this particle has the above dispersion relation and moves in the ground state.

Now, if we were to flip a second spin, this would be equivalent to two magnons moving in the chain. Let the positions and momenta of the two excitations be $n_{1}, n_{2}$ and $p_{1}, p_{2}$ respectively, and define $n_{1}<n_{2}$ without loss of generality. If $n_{1}<<n_{2}$, that is, if the magnons are far away from each other, they shouldn't interfere with the other since we are considering only nearest-neighbour interactions. However, when they come closer to each other, the Hamiltonian will act differently on the chain. We write

$$
\left|p_{1}, p_{2}\right\rangle=\sum_{n_{1}<n_{2}=1}^{N}\left[e^{i\left(p_{1} n_{1}+p_{2} n_{2}\right)}+S\left(p_{1}, p_{2}\right) e^{i\left(p_{1} n_{2}+p_{2} n_{1}\right)}\right] S_{n_{1}}^{-} S_{n_{2}}^{-}|0\rangle
$$

We can interchange the labels in the second term and split the sum into two, giving

$$
\left|p_{1}, p_{2}\right\rangle=\left[\sum_{n_{1}<n_{2}} e^{i\left(p_{1} n_{1}+p_{2} n_{2}\right)}+\sum_{n_{2}<n_{1}} S\left(p_{1}, p_{2}\right) e^{i\left(p_{1} n_{1}+p_{2} n_{2}\right)}\right] S_{n_{1}}^{-} S_{n_{2}}^{-}|0\rangle
$$

Thus, the terms describe two different regions - one in which the first magnon is to the left and the second in which the second magnon is to the left. Clearly, in the latter case, there is the added factor $S\left(p_{1}, p_{2}\right)$. This is equivalent to saying that when they scatter with each other, a phase shift occurs. 
When $n_{1}$ and $n_{2}$ and their nearest neighbours aren't overlapping, that is, when $n_{1}>n_{2}+2$, the magnons behave like free particles completely unaffected by each other and the eigenvalue with respect to the Hamiltonian is simply $E\left(p_{1}\right)+E\left(p_{2}\right)$.

If this isn't the case and the down spins are adjacent, we have many more non-zero terms. These impose the constraint on $S\left(p_{1}, p_{2}\right)$ that

$$
S\left(p_{1}, p_{2}\right)=\frac{\cot \frac{p_{1}}{2}-\cot \frac{p_{2}}{2}-2 i}{\cot \frac{p_{1}}{2}-\cot \frac{p_{2}}{2}+2 i}
$$

Let $\lambda=2 \cot \frac{p}{2}$. Then,

$$
\Longrightarrow e^{i p}=\frac{\lambda+i / 2}{\lambda-i / 2}
$$

The S-matrix then becomes

$$
S\left(p_{1}, p_{2}\right)=\frac{\lambda_{1}-\lambda_{2}-i}{\lambda_{1}-\lambda_{2}+i}
$$

Since this can be written in the form $S\left(p_{1}, p_{2}\right)=e^{i \theta}, S$ is the scattering matrix between the two magnons. Clearly,

$$
S\left(p_{1}, p_{2}\right) S\left(p_{2}, p_{1}\right)=\frac{\cot \frac{p_{1}}{2}-\cot \frac{p_{2}}{2}-2 i}{\cot \frac{p_{1}}{2}-\cot \frac{p_{2}}{2}+2 i} \cdot \frac{\cot \frac{p_{1}}{2}-\cot \frac{p_{2}}{2}+2 i}{\cot \frac{p_{1}}{2}-\cot \frac{p_{2}}{2}-2 i}=1
$$

Using the condition $a(n+N)=a(n)$, we have the following revised periodicity conditions.

$$
\begin{aligned}
& S\left(p_{1}, p_{2}\right) e^{i p_{1} N}=1 \Longrightarrow e^{i p_{1} N}=\frac{1}{S\left(p_{1}, p_{2}\right)}=S\left(p_{2}, p_{1}\right) \\
& S\left(p_{2}, p_{1}\right) e^{i p_{2} N}=1 \Longrightarrow e^{i p_{2} N}=\frac{1}{S\left(p_{2}, p_{1}\right)}=S\left(p_{1}, p_{2}\right)
\end{aligned}
$$

Using equations (3.14) and (3.15), the above equations become

$$
\begin{aligned}
& \left(\frac{\lambda_{1}+i / 2}{\lambda_{1}-i / 2}\right)^{N}=\frac{\lambda_{1}-\lambda_{2}+i}{\lambda_{1}-\lambda_{2}-i} \\
& \left(\frac{\lambda_{2}+i / 2}{\lambda_{2}-i / 2}\right)^{N}=\frac{\lambda_{2}-\lambda_{1}+i}{\lambda_{2}-\lambda_{1}-i}
\end{aligned}
$$


The above two equations are called the Bethe Ansatz Equations (BAE). The parameters $\lambda_{1}$ and $\lambda_{2}$, which we call the rapidities, are directly related to the momenta by equation (3.14). Hence, the BAE essentially quantise the rapidities and, therefore, the momenta. These equations give the quantisation condition for which the plain-wave ansatz holds.

When we flip more than two spins, say $l$ spins, we generate $l$ magnons. However, the BAE have a similar form. For the $i$ th magnon, we have

$$
\left(\frac{\lambda_{i}+i / 2}{\lambda_{i}-i / 2}\right)^{N}=\prod_{i \neq j}^{l} \frac{\lambda_{i}-\lambda_{j}+i}{\lambda_{i}-\lambda_{j}-i}
$$

and there are $l$ such equations which give all the constraints on the state.

It can also be noted that the energy of the $i$ th particle is

$$
E_{i}=\frac{1}{2} \frac{J}{\lambda^{2}+1 / 4}
$$

Thus, using the so-called 'Coordinate Bethe Ansatz' technique, we have obtained an expression for the energy of the quasiparticles generated by flipping a spin and also a set of quantisation conditions, the Bethe Ansatz Equations, on the momenta of these particles. While the Coordinate Bethe Ansatz provides a very transparent view of the physical aspects of the system, it doesn't highlight the integrability inherent in the model. By using a technique called the Algebraic Bethe Ansatz formulated by L.D. Faddeev, we will see that these aspects will be more pronounced while the exact same relations will be obtained.

\subsection{Algebraic Bethe Ansatz}

For the system to be integrable, we need to show that there are $N$ mutually commuting operators, one of which, as we will see, is the Hamiltonian. The Lax operator is a generator for these operators. In the case of our model, we define the Lax operator to be acting on both the quantum space and what we now define to be an auxiliary space $V=\mathcal{C}^{2}$ in the spin- $1 / 2$ case. We define it below and will see how it enables us to generate these commuting operators.

$$
L_{n, a}(\lambda)=\lambda I_{n} \otimes I_{a}+i \sum_{\alpha} S_{n}^{\alpha} \otimes \sigma^{\alpha}
$$

Here, $I_{n}$ and $I_{a}$ are the identity operators in the quantum and auxiliary spaces respectively and are both equal to $I_{2}$ in the spin-1/2 case. $\lambda$ is called the spectral parameter, and is a complex 
number. The Lax operator can also be written as a $2 \times 2$ matrix acting in the auxiliary space with its elements acting in the quantum space, in which case it would have the following form.

$$
L_{n, a}(\lambda)=\left(\begin{array}{cc}
\lambda+i S_{n}^{3} & i S_{n}^{-} \\
i S_{n}^{+} & \lambda-i S_{n}^{3}
\end{array}\right)
$$

where $S_{n}^{ \pm}=S_{n}^{1} \pm i S_{n}^{2}$. The auxiliary space is simply interpreted to be the space from which the matrix structure of the Lax operator is obtained.

The Lax operator can also be rewritten in terms of the permutation operator, which acts in $\mathcal{C}^{2} \otimes \mathcal{C}^{2}$ and is given by

$$
P=\frac{1}{2}\left(I \otimes I+i \sum_{\alpha} \sigma^{\alpha} \otimes \sigma^{\alpha}\right)
$$

The permutation operator swaps the order of two spins. That is,

$$
P(|x\rangle \otimes|y\rangle)=|y\rangle \otimes|x\rangle
$$

where $|x\rangle$ and $|y\rangle$ can be up or down spins.

Writing the Lax operator in terms of the permutation operator, we get

$$
L_{n, a}(\lambda)=\left(\lambda-\frac{i}{2}\right) I_{n, a}+i P_{n, a}
$$

The next step is to define the commutation relations that the Lax operator obeys, which will prove to be useful in later derivations.

\subsubsection{Fundamental Commutation Relations}

Consider two Lax operators, $L_{n, a_{1}}(\lambda)$ and $L_{n, a_{2}}(\mu)$ acting in different auxiliary spaces $V_{1}$ and $V_{2}$, but the same quantum space. The products of these operators $L_{n, a_{1}}(\lambda) L_{n, a_{2}}(\mu)$ and $L_{n, a_{2}}(\mu) L_{n, a_{1}}(\lambda)$ are defined in $h \otimes V_{1} \otimes V_{2}$, and can be shown to not commute.

In order to obtain a relation with the above products, we introduce an operator $R_{a_{1}, a_{2}}(\lambda-\mu)$ acting in $V \otimes V$ which is defined such that

$$
R_{a_{1}, a_{2}}(\lambda-\mu) L_{n, a_{1}}(\lambda) L_{n, a_{2}}(\mu)=L_{n, a_{2}}(\mu) L_{n, a_{1}}(\lambda) R_{a_{1}, a_{2}}(\lambda-\mu)
$$


This relation is called the RLL relation and we find that the operator has the form

$$
R_{a_{1}, a_{2}}(\lambda)=\lambda I_{a_{1}, a_{2}}+i P_{a_{1}, a_{2}}
$$

which can be shown using the explicit forms of the Lax and permutation operators in conjunction with the following relations involving the permutation operator. The $R$-matrix is interpreted to be the scattering matrix.

$$
\begin{aligned}
& P_{n, a_{1}} P_{n, a_{2}}=P_{a_{1}, a_{2}} P_{n, a_{1}}=P_{n, a_{2}} P_{a_{2}, a_{1}} \\
& P_{n, a_{2}} P_{n, a_{1}}=P_{a_{2}, a_{1}} P_{n, a_{2}}=P_{n, a_{1}} P_{a_{1}, a_{2}}
\end{aligned}
$$

and

$$
P_{a_{1}, a_{2}}=P_{a_{2}, a_{1}}
$$

These relations can be easily derived by operating these permutation operator products on an arbitrary vector.

The proof that the RLL relation holds with $R_{a_{1}, a_{2}}(\lambda-\mu)$ is given in (A.1).

The Lax operator has an interpretation of being a discrete connection between neighbouring sites. The Lax connection below corresponds to a connection from site $n$ to $n+1$ along the chain and arises from a discretisation of the Dirac equation.

$$
\psi_{n+1}=L_{n} \psi_{n}
$$

where $\psi_{n}=\left(\begin{array}{c}\psi_{n}^{1} \\ \psi_{n}^{2}\end{array}\right)$ with entries in $H$.

The ordered product of all Lax operators from $n_{1}$ to $n_{2}+1$

$$
T_{n_{1}, a}^{n_{2}}(\lambda)=L_{n_{2}, a}(\lambda) \ldots L_{n_{1}, a}(\lambda)
$$

defines transport from $n_{1}$ to $n_{2}+1$ along the chain.

The full product of all Lax operators from 1 to $N$ is, therefore, a monodromy around the circular chain and is given by

$$
T_{N, a}(\lambda)=L_{N, a}(\lambda) \ldots L_{1, a}(\lambda)
$$

Once again, we see that the products of two different monodromies do not commute. However, the same expression for $R_{a_{1}, a_{2}}(\lambda-\mu)$ from before can be used to establish what is known as the $\mathrm{RTT}$ relation and is given by 


$$
R_{a_{1}, a_{2}}(\lambda-\mu) T_{a_{1}}(\lambda) T_{a_{2}}(\mu)=T_{a_{2}}(\mu) T_{a_{1}}(\lambda) R_{a_{1}, a_{2}}(\lambda-\mu)
$$

Here, the index $\mathrm{N}$ has been dropped, temporarily.

Using the shorthand notation $R_{a_{1}, a_{2}}(\lambda-\mu)=R_{12}, L_{i, a_{1}}(\lambda)=L_{1}^{(i)}$, and $L_{j, a_{2}}(\mu)=L_{2}^{(j)} \Longrightarrow$ $T_{a_{1}}(\lambda)=L_{1}^{(N)} \ldots L_{1}^{(1)}, T_{a_{2}}(\mu)=L_{2}^{(N)} \ldots L_{2}^{(1)}$ for convenience, we see that the RTT relation holds since

$$
\begin{aligned}
R_{a_{1}, a_{2}}(\lambda-\mu) & T_{a_{1}}(\lambda) T_{a_{2}}(\mu) \\
& =R_{12} L_{1}^{(N)} \ldots L_{1}^{(1)} L_{2}^{(N)} \ldots L_{2}^{(1)} \\
& =R_{12} L_{1}^{(N)} \ldots L_{1}^{(2)} L_{2}^{(N)} L_{1}^{(1)} L_{2}^{(N-1)} \ldots L_{2}^{(1)}\left(\text { since }\left[L_{1}, L_{2}^{(N)}\right]=0\right) \\
& =L_{2}^{\prime} L_{1}^{\prime} R_{12} L_{1} L_{2}\left(\text { RLL relation for } R_{12}, L_{1}^{\prime} \text { and } L_{2}^{\prime}\right) \\
& =L_{2}^{\prime} L_{1}^{\prime} L_{2} L_{1} R_{12}\left(\text { RLL relation for } R_{12}, L_{1} \text { and } L_{2}\right) \\
& =L_{2}^{\prime} L_{2} L_{1}^{\prime} L_{1} R_{12}\left(\text { since }\left[L_{2}, L_{1}^{\prime}\right]=0\right) \\
& =T_{a_{2}}(\mu) T_{a_{1}}(\lambda) R_{a_{1}, a_{2}}(\lambda-\mu)
\end{aligned}
$$

\subsubsection{Monodromy and the Family of the Transfer Matrix}

The Lax operator can be written as a $2 \times 2$ matrix acting in the auxilliary space, as shown below.

$$
\begin{aligned}
& L_{n, a}(\lambda)=\lambda I_{n} \otimes I_{a}+i \sum_{\alpha} S_{n}^{\alpha} \otimes \sigma^{\alpha} \\
& \Longrightarrow L_{n, a}(\lambda)=\left(\begin{array}{cc}
\lambda+i S_{n}^{3} & i S_{n}^{-} \\
i S_{n}^{+} & \lambda-i S_{n}^{3}
\end{array}\right)
\end{aligned}
$$

Using this expansion, the monodromy, which is the product of all Lax operators along the chain, is seen to be a polynomial of order $N$

$$
T_{N, a}(\lambda)=\lambda^{N}+i \lambda^{N-1} \sum_{\alpha} S^{\alpha} \otimes \sigma^{\alpha}+\ldots
$$

The total spin $S^{\alpha}$ appears as a coefficient in the term of order $N-1$. 
Much like the Lax operator, the monodromy can be written as a 2 x 2 matrix in the auxillary space

$$
T_{N, a}(\lambda)=\left(\begin{array}{ll}
A_{N}(\lambda) & B_{N}(\lambda) \\
C_{N}(\lambda) & D_{N}(\lambda)
\end{array}\right)
$$

We define the trace of the monodromy or the transfer matrix to be a function

$$
F(\lambda)=\operatorname{tr} T(\lambda)=A(\lambda)+D(\lambda)
$$

In general, it can be shown that this family of operators commutes with itself. In order to show that this is true, we assert that $R_{a_{1}, a_{2}}(\lambda)=\lambda I+i P$ has an inverse of the form

$$
R_{a_{1}, a_{2}}^{-1}(\lambda)=\frac{1}{\lambda^{2}+1}(\lambda I-i P)
$$

Using the fact that $P^{2}=I$, we see that this is clearly true, since

$$
R_{a_{1}, a_{2}}(\lambda) R_{a_{1}, a_{2}}^{-1}(\lambda)=\frac{1}{\lambda^{2}+1}(\lambda I-i P)(\lambda I+i P)=\frac{1}{\lambda^{2}+1}\left(\lambda^{2}+1\right) I=R_{a_{1}, a_{2}}^{-1}(\lambda) R_{a_{1}, a_{2}}(\lambda)
$$

The RTT relation gives us $R_{12} T_{1} T_{2}=T_{2} T_{1} R_{12}$. Pre-multiplying both sides by $R_{12}^{-1}$ gives

$$
\begin{aligned}
T_{1} T_{2}= & R_{12}^{-1} T_{2} T_{1} R_{12} \\
\Longrightarrow & \operatorname{tr}_{a_{1}, a_{2}}\left(T_{1} T_{2}\right)=t r_{a_{1}, a_{2}}\left(R_{12}^{-1} T_{2} T_{1} R_{12}\right)=t r_{a_{1}, a_{2}}\left(T_{2} T_{1} R_{12} R_{12}^{-1}\right)=t r_{a_{1}, a_{2}}\left(T_{2} T_{1}\right) \\
& \Longrightarrow t r_{a_{1}, a_{2}}\left(T_{1} T_{2}\right)=t r_{a_{1}, a_{2}}\left(T_{2} T_{1}\right) \Longrightarrow t r_{a_{1}}\left(T_{1}\right) t r_{a_{2}}\left(T_{2}\right)=t r_{a_{2}}\left(T_{2}\right) t r_{a_{1}}\left(T_{1}\right) \\
& \Longrightarrow F(\lambda) F(\mu)=F(\mu) F(\lambda) \Longrightarrow[F(\lambda), F(\mu)]=0
\end{aligned}
$$

So, we clearly have

$$
[F(\lambda), F(\mu)]=0
$$

The above proof follows from the cyclic property of traces of mutually commuting matrix products. 
The nontrivial $\lambda$ expansion of $F(\lambda)$ can be deduced from the monodromy matrix to be

$$
F(\lambda)=2 \lambda^{N}+\sum_{l=0}^{N-2} Q_{l} \lambda^{l}
$$

The $N-1$ operators $\left\{Q_{l}\right\}$ are commuting, as follows from the commutativity of the trace itself.

In order to find the momentum and hence the Hamiltonian, we try to find the unit shift operator in terms of known operators.

By knowing the value and first derivative of the Lax operator at a point, the expansion of $F(\lambda)$ can be controlled.

From equation (3.27), the derivative of the Lax operator is seen to be

$$
\frac{d}{d \lambda} L_{n, a}(\lambda)=I_{n, a}
$$

for all $\lambda$.

It also follows that, at $\lambda=\frac{i}{2}$

$$
L_{n, a}(i / 2)=i P_{n, a}
$$

Using the above equation in the expression for monodromy, we get

$$
T_{N, a}(i / 2)=i^{N} P_{N, a} P_{N-1, a} \ldots P_{1, a}
$$

This sequence of permutations operating on a state vector corresponds to moving each component one place to the right i.e. the unit shift operation. This is equivalent to the sequence $P_{1,2} P_{2,3} \ldots P_{N-1, N} P_{N, a}$.

Using equation (3.25), the trace of the permutation operator $P_{N, a}$ over the auxillary space is found to be

$$
\operatorname{tr}_{a} P_{N, a}=I_{N}
$$

while the trace of the other permutation operators is trivially 0 .

We define the shift operator in $\mathcal{H}$ to be

$$
U=i^{-N} \operatorname{tr}_{a} T_{N}(i / 2)=P_{1,2} P_{2,3} \ldots P_{N-1, N}
$$

For any operator $X_{n}=\left(\prod_{i=1}^{n-1} \otimes I\right) \otimes X \otimes\left(\prod_{j=n+1}^{N} \otimes I\right) \otimes I$, we have 


$$
P_{n_{1}, n_{2}} X_{n_{2}} P_{n_{1}, n_{2}}=X_{n_{1}}
$$

This can easily be seen by operating on a state vector belonging to $\mathcal{H} \otimes V$, say,

$$
\begin{gathered}
\Omega=\left(\prod_{j} \otimes v_{j}\right) \otimes w \\
\Longrightarrow P_{n_{1}, n_{2}} X_{n_{2}} P_{n_{1}, n_{2}} \Omega=P_{n_{1}, n_{2}} X_{n_{2}}\left(\prod_{j=1}^{n_{1}-1} \otimes v_{j}\right) \otimes v_{n_{2}} \otimes\left(\prod_{j=n_{1}+1}^{n_{2}-1} \otimes v_{j}\right) \otimes v_{n_{1}} \otimes w \\
=P_{n_{1}, n_{2}}\left(\prod_{j=1}^{n_{1}-1} \otimes v_{j}\right) \otimes v_{n_{2}} \otimes\left(\prod_{j=n_{1}+1}^{n_{2}-1} \otimes v_{j}\right) \otimes X v_{n_{1}} \otimes w \\
=P_{n_{1}, n_{2}}\left(\prod_{j=1}^{n_{1}-1} \otimes v_{j}\right) \otimes X v_{n_{1}} \otimes\left(\prod_{j=n_{1}+1}^{n_{2}-1} \otimes v_{j}\right) \otimes v_{n_{2}} \otimes w \\
=X_{n_{1}} \Omega
\end{gathered}
$$

Therefore, we have

$$
\begin{gathered}
X_{n} U=X_{n} P_{1,2} P_{2,3} \ldots P_{N-1, N}=P_{1,2} P_{2,3} \ldots X_{n} P_{n-1, n} P_{n, n+1} \ldots P_{N-1, N} \\
\quad\left[\text { since } X_{n} \text { commutes with all permutations not involving } n\right] \\
=P_{1,2} P_{2,3} \ldots X_{n} P_{n-1, n} X_{n-1} P_{n, n+1} \ldots P_{N-1, N}=U X_{n-1}
\end{gathered}
$$$$
\text { [which follows from equation (3.49)] }
$$

Hence,

$$
X_{n} U=U X_{n-1}
$$

The operator $\mathrm{U}$ is unitary i.e. $U U^{*}=U^{*} U=I$, since

$$
\begin{gathered}
U U^{*}=P_{1,2} P_{2,3} \ldots P_{N-1, N} P_{N-1, N}^{*} P_{N-2, N-1}^{*} \ldots P_{1,2} \\
=P_{1,2} P_{2,3} \ldots P_{N-2, N-1} P_{N-1, N} P_{N-1, N} P_{N-2, N-1} \ldots P_{2,3} P_{1,2}=I \\
\\
{\left[\text { since } P^{*}=P \text { and } P^{2}=I\right]}
\end{gathered}
$$

Interpreting $U$ as a unit shift operator to the right and its inverse $U^{-1}$ as a unit shift operator to the left, it is apparent that $U^{-1} X_{n} U=X_{n-1}$

This allows us to introduce momentum in terms of $U$. Since it produces an infinitesimal shift, or a unit shift on the lattice,

$$
e^{i P}=U
$$


Since we know the nature of the Lax operator and its first derivative around $\lambda=i / 2$, we can expand the derivative of the monodromy's trace.

First, we note that the derivative of the monodromy is

$$
\begin{aligned}
\left.\frac{d}{d \lambda} T_{a}(\lambda)\right|_{\lambda=i / 2} & =\left.\sum_{n} L_{N, a}(\lambda) \ldots \frac{d}{d \lambda} L_{n, a}(\lambda) \ldots L_{1, a}(\lambda)\right|_{\lambda=i / 2} \\
& =\sum_{n} i P_{N, a}(\lambda) \ldots I_{n, a} \ldots i P_{1, a}(\lambda)
\end{aligned}
$$

Hence,

$$
\left.\frac{d}{d \lambda} T_{a}(\lambda)\right|_{\lambda=i / 2}=i^{N-1} \sum_{n} P_{N, a} \ldots \widehat{P_{n, a}} \ldots P_{1, a}
$$

where the hat implies that the factor is absent. Using the above equation and rewriting the string of permutations as done before, we can see that

$$
\left.\frac{d}{d \lambda} F_{a}(\lambda)\right|_{\lambda=i / 2}=\left.\frac{d}{d \lambda} \operatorname{tr}_{a} T_{N, a}(\lambda)\right|_{\lambda=i / 2}=i^{N-1} \sum_{n} P_{1,2} P_{2,3} \ldots P_{n-1, n+1} \ldots P_{N-1, N} .
$$

since the trace of $T_{N, a}$ is the identity operator.

Multiplying by $F_{a}(\lambda)^{-1}$, we get

$$
\begin{gathered}
\left.\frac{d}{d \lambda} F_{a}(\lambda) F_{a}(\lambda)^{-1}\right|_{\lambda=i / 2}=\left.\frac{d}{d \lambda} \ln F_{a}(\lambda)\right|_{\lambda=i / 2} \\
=\left(i^{N-1} \sum_{n} P_{1,2} P_{2,3} \ldots P_{n-1, n+1} \ldots P_{N-1, N}\right)\left(i^{-N} P_{N, N-1} \ldots P_{n+1, n} P_{n, n-1} \ldots P_{3,2} P_{2,1}\right)
\end{gathered}
$$

Hence,

$$
\left.\frac{d}{d \lambda} \ln F_{a}(\lambda)\right|_{\lambda=i / 2}=\frac{1}{i} \sum_{n} P_{1,2} \ldots P_{n-2, n-1}\left(P_{n-1, n+1} P_{n+1, n} P_{n, n-1}\right) \ldots P_{2,1}
$$

[all the terms from $P_{n+1, n+2}$ and above get squared and become the identity operator]

As follows, it can be shown that $P_{n-1, n+1} P_{n+1, n} P_{n, n-1}=P_{n, n+1}$.

$$
\begin{gathered}
P_{n-1, n+1} P_{n+1, n} P_{n, n-1} \Omega=P_{n-1, n+1} P_{n+1, n}\left(\prod_{j=1}^{n-2} \otimes v_{j}\right) \otimes v_{n} \otimes v_{n-1} \otimes v_{n+1} \otimes\left(\prod_{j=n+2}^{N} \otimes v_{j}\right) \\
=P_{n-1, n+1}\left(\prod_{j=1}^{n-2} \otimes v_{j}\right) \otimes v_{n} \otimes v_{n+1} \otimes v_{n-1} \otimes\left(\prod_{j=n+2}^{N} \otimes v_{j}\right) \\
=\left(\prod_{j=1}^{n-2} \otimes v_{j}\right) \otimes v_{n-1} \otimes v_{n+1} \otimes v_{n} \otimes\left(\prod_{j=n+2}^{N} \otimes v_{j}\right) \\
=P_{n, n+1} \Omega
\end{gathered}
$$

Hence, using the above result in equation (3.53), we get

$$
\begin{gathered}
\left.\frac{d}{d \lambda} \ln F_{a}(\lambda)\right|_{\lambda=i / 2}=\frac{1}{i} \sum_{n} P_{1,2} \ldots P_{n-2, n-1}\left(P_{n, n+1}\right) P_{n-1, n-2} \ldots P_{2,1} \\
=\frac{1}{i} \sum_{n} P_{1,2} \ldots P_{n-2, n-1} P_{n-1, n-2} \ldots P_{2,1} P_{n, n+1}
\end{gathered}
$$


[since $P_{n, n+1}$ commutes with every other factor in the expression]

$$
=\frac{1}{i} \sum_{n} P_{n, n+1}
$$

[since all the other terms get squared and become the identity operator]

Hence, we have

$$
\left.\frac{d}{d \lambda} \ln F_{a}(\lambda)\right|_{\lambda=i / 2}=\frac{1}{i} \sum_{n} P_{n, n+1}
$$

We know that $P_{n, n+1}=\frac{1}{2}\left(I+4 \sum_{\alpha} S_{n}^{\alpha} S_{n+1}^{\alpha}\right) \Longrightarrow \sum_{\alpha} S_{n}^{\alpha} S_{n+1}^{\alpha}=\frac{1}{2} P_{n, n+1}-\frac{1}{4} I$

Using the original expression for the Hamiltonian of the spin chain system, we have

$$
\begin{aligned}
H & =\sum_{n} \sum_{\alpha}\left(S_{n}^{\alpha} S_{n+1}^{\alpha}-\frac{1}{4}\right) \\
= & \sum_{n}\left(\frac{1}{2} P_{n, n+1}-\frac{1}{4}-\frac{1}{4}\right) \\
& =\sum_{n}\left(\frac{1}{2} P_{n, n+1}-\frac{1}{2}\right) \\
& =\frac{1}{2} \sum_{n} P_{n, n+1}-\frac{N}{2}
\end{aligned}
$$

Substituting the expression in equation (3.54) gives us the final result for the Hamiltonian in terms of the trace operator.

$$
H=\left.\frac{i}{2} \frac{d}{d \lambda} \ln F(\lambda)\right|_{\lambda=i / 2}-\frac{N}{2}
$$

Clearly, the Hamiltonian belongs to the family of $N-1$ commuting operators associated with the trace. Adding the $S^{3}$ component of spin to this family completes it to have $N$ commuting operators. Hence, the spin chain model, in this description, is integrable with $N$ degrees of freedom.

\subsubsection{Deriving the Bethe Ansatz Equations}

As shown in the following section, the Bethe Ansatz Equations (BAE) provide a constraint on the parameters $\left\{\lambda_{i}\right\}$ that are found to characterise states in the spin chain model. In the XXX case, these constraint equations were derived in the Algebraic Bethe Ansatz formalism by Faddeev, who described a procedure to diagonalise the family of operators $F(\lambda)$.

To do this, we first need to understand the action of each element of the monodromy matrix and the implications they have. Writing the monodromy $T_{N, a}(\lambda)$ as a $2 \times 2$ matrix in the auxiliary space $V$ like in equation (3.37) and using the representation of the R-matrix like in equation (3.29), we can explicitly write each term in the RTT relation as $4 \times 4$ matrices in the $V \otimes V$ 
space, as shown in detail in Appendix (A.2). Equating both sides of the relation, we get 16 relations between $A(\lambda), B(\lambda), C(\lambda)$, and $D(\lambda)$.

The three necessary FCR are as follows for the aforementioned procedure as follows.

$$
\begin{gathered}
{[B(\lambda), B(\mu)]=0} \\
A(\lambda) B(\mu)=f(\lambda-\mu) B(\mu) A(\lambda)+g(\lambda-\mu) B(\lambda) A(\mu) \\
D(\lambda) B(\mu)=h(\lambda-\mu) B(\mu) D(\lambda)+k(\lambda-\mu) B(\lambda) D(\mu)
\end{gathered}
$$

where the coefficients are defined as

$$
f(\lambda)=\frac{\lambda-i}{\lambda}, \quad g(\lambda)=\frac{i}{\lambda}, \quad h(\lambda)=\frac{\lambda+i}{\lambda}, \quad k(\lambda)=\frac{-i}{\lambda}
$$

We now define a reference highest weight state $\Omega$, such that $C(\lambda) \Omega=0$. In order to find the state, we note that, for a particular Lax operator $L_{n, a}(\lambda)$ in any subspace, there exists a vector $\omega_{n}$ such that the Lax operator's action on it effectively becomes a triangular matrix in the auxiliary space. That is,

$$
L_{n, a}(\lambda) \omega_{n}=\left(\begin{array}{cc}
\lambda+\frac{i}{2} & * \\
0 & \lambda-\frac{i}{2}
\end{array}\right) \omega_{n}
$$

Here, ${ }^{*}$ is the element of the matrix which is irrelevant to further analyses. This vector is found to be given by $\left(\begin{array}{l}1 \\ 0\end{array}\right)$.

Hence, the highest weight state is a tensor product of $\omega_{n}$ across all subspaces.

$$
\Omega=\prod_{n} \otimes \omega_{n}
$$

By acting on $\Omega$ with the monodromy operator, which is the product of Lax operators, we obtain

$$
T(\lambda) \Omega=\left(\begin{array}{cc}
\left(\lambda+\frac{i}{2}\right)^{N} & * \\
0 & \left(\lambda+\frac{i}{2}\right)^{N}
\end{array}\right) \Omega=\left(\begin{array}{cc}
\alpha^{N}(\lambda) & * \\
0 & \delta^{N}(\lambda)
\end{array}\right) \Omega
$$

where $\alpha(\lambda)=\lambda+\frac{i}{2}$ and $\delta(\lambda)=\lambda-\frac{i}{2}$. 
Using equation (3.37), we see that

$$
A(\lambda) \Omega=\alpha^{N}(\lambda) \Omega, \quad C(\lambda) \Omega=0, \quad D(\lambda) \Omega=\delta^{N}(\lambda) \Omega
$$

which shows that $\Omega$ is a simultaneous eigenstate of $A(\lambda), D(\lambda)$ and $F(\lambda)=A(\lambda)+D(\lambda)$.

The next goal is to find the form of remaining states and the eigenvalues obtained upon the action of $F(\lambda)$. By drawing a comparison between the spin chain model and the harmonic oscillator, we see that $\Omega$ corresponds to the vacuum state of the harmonic oscillator. Correspondingly, we act on this reference state with $B(\lambda)$ to generate other states. Hence, a general state is given by

$$
\Phi(\{\lambda\})=B\left(\lambda_{1}\right) B\left(\lambda_{2}\right) \ldots B\left(\lambda_{l}\right) \Omega
$$

$B(\lambda)$ is, in a sense, a raising operator, although the values of $\{\lambda\}$ for which $\Phi(\{\lambda\})$ is an eigenstate of $F(\lambda)$ cannot be arbitrary. The constraint equations on these parameters will turn out to be the BAE, as discussed below.

Assuming that $\Phi(\{\lambda\})$ is an eigenstate of $F(\lambda)$ for a particular case of $\lambda_{1}, \lambda_{2} \ldots \lambda_{l}$, we have

$$
F(\lambda) \Phi(\{\lambda\})=(A(\lambda)+D(\lambda)) \Phi(\lambda)=\Lambda(\lambda, \lambda) \Phi(\{\lambda\})
$$

We have

$\left.A(\lambda) \Phi(\{\lambda\})=\prod_{k=1}^{l} f\left(\lambda-\lambda_{k}\right) \alpha^{N}(\lambda) B\left(\lambda_{1}\right) B\left(\lambda_{2}\right) \ldots B\left(\lambda_{l}\right) \Omega+\sum_{k=1}^{l} M_{k}(\lambda,\{\lambda\}) B\left(\lambda_{1}\right) B\left(\lambda_{2}\right) \ldots \widehat{B\left(\lambda_{k}\right.}\right) \ldots B\left(\lambda_{l}\right) \Omega$

where

$$
M_{j}(\lambda,\{\lambda\})=g\left(\lambda-\lambda_{j}\right) \prod_{k=j}^{l} f\left(\lambda-\lambda_{j}\right) \alpha^{N}\left(\lambda_{j}\right)
$$

and 
$\left.D(\lambda) \Phi(\{\lambda\})=\prod_{k=1}^{l} h\left(\lambda-\lambda_{k}\right) \delta^{N}(\lambda) B\left(\lambda_{1}\right) B\left(\lambda_{2}\right) \ldots B\left(\lambda_{l}\right) \Omega+\sum_{k=1}^{l} N_{k}(\lambda,\{\lambda\}) B\left(\lambda_{1}\right) B\left(\lambda_{2}\right) \ldots \widehat{B\left(\lambda_{k}\right.}\right) \ldots B\left(\lambda_{l}\right) \Omega$

where

$$
N_{j}(\lambda,\{\lambda\})=k\left(\lambda-\lambda_{j}\right) \prod_{k=j}^{l} h\left(\lambda-\lambda_{j}\right) \delta^{N}\left(\lambda_{j}\right)
$$

which can be proved by induction, as shown in Appendix (A.3).

Since we now know how $A(\lambda)$ and $D(\lambda)$ interact with a vector state, we can see that

$$
\begin{aligned}
F(\lambda) \Phi(\lambda)=\prod_{k=1}^{l} f\left(\lambda-\lambda_{k}\right) \alpha^{N}(\lambda) \Phi(\{\lambda\})+ \\
\left.\sum_{k=1}^{l} M_{k}(\lambda,\{\lambda\}) B\left(\lambda_{1}\right) B\left(\lambda_{2}\right) \ldots \widehat{B\left(\lambda_{k}\right.}\right) \ldots B\left(\lambda_{l}\right) \Omega+\prod_{k=1}^{l} h\left(\lambda-\lambda_{k}\right) \delta^{N}(\lambda) \Phi(\{\lambda\})+ \\
\left.\sum_{k=1}^{l} N_{k}(\lambda,\{\lambda\}) B\left(\lambda_{1}\right) B\left(\lambda_{2}\right) \ldots \widehat{B\left(\lambda_{k}\right.}\right) \ldots B\left(\lambda_{l}\right) \Omega
\end{aligned}
$$

This can be rewritten as

$$
F(\lambda) \Phi(\lambda)=\left[\prod_{k=1}^{l} f\left(\lambda-\lambda_{k}\right) \alpha^{N}(\lambda)+\prod_{k=1}^{l} h\left(\lambda-\lambda_{k}\right) \delta^{N}(\lambda)\right] \Phi(\{\lambda\})=\Lambda(\lambda,\{\lambda\}) \Phi(\{\lambda\})
$$

provided that

$$
\begin{gathered}
M_{j}(\lambda,\{\lambda\})=-N_{j}(\lambda,\{\lambda\}) \Longrightarrow g\left(\lambda-\lambda_{j}\right) \prod_{k \neq j}^{l} f\left(\lambda_{j}-\lambda_{k}\right) \alpha^{N}\left(\lambda_{j}\right)=-k\left(\lambda-\lambda_{j}\right) \prod_{k \neq j}^{l} h\left(\lambda_{j}-\lambda_{k}\right) \delta^{N}\left(\lambda_{j}\right) \\
\Longrightarrow \prod_{j \neq k}^{l} f\left(\lambda-\lambda_{j}\right) \alpha^{N}\left(\lambda_{k}\right)=\prod_{j \neq k}^{l} h\left(\lambda-\lambda_{j}\right) \delta^{N}\left(\lambda_{k}\right)
\end{gathered}
$$




$$
\Longrightarrow \frac{\alpha^{N}\left(\lambda_{j}\right)}{\delta^{N}\left(\lambda_{j}\right)}=\prod_{k \neq j}^{l} \frac{h\left(\lambda_{j}-\lambda_{k}\right)}{f\left(\lambda_{j}-\lambda_{k}\right)} \Longrightarrow\left(\frac{\lambda_{j}+\frac{i}{2}}{\lambda_{j}-\frac{i}{2}}\right)^{N}=\prod_{k \neq j}^{l} \frac{\lambda_{j}-\lambda_{k}+i}{\lambda_{j}-\lambda_{k}} \cdot \frac{\lambda_{j}-\lambda_{k}}{\lambda_{j}-\lambda_{k}-i}
$$

Hence, we have

$$
\left(\frac{\lambda_{j}+\frac{i}{2}}{\lambda_{j}-\frac{i}{2}}\right)^{N}=\prod_{k \neq j}^{l} \frac{\lambda_{j}-\lambda_{k}+i}{\lambda_{j}-\lambda_{k}-i}
$$

which defines the constraints on $\{\lambda\}$ in order for a particular vector state to be an eigenstate of the trace of the monodromy and hence, of the Hamiltonian. Therefore, the above are the Bethe Ansatz Equations (BAE).

In order to find the momentum, spin and energy eigenvalues of the Bethe vector state, we use the definitions from equations (3.48) and (3.51) to get

$$
\begin{gathered}
e^{i P}=U \Longrightarrow i P=\log U=\log \left(i^{-N} t r_{a} T\left(\frac{i}{2}\right)\right) \\
\Longrightarrow P=\frac{1}{i} \log \left(i^{-N} t r_{a} T\left(\frac{i}{2}\right)\right)
\end{gathered}
$$

From equation (3.71), we have

$$
\begin{aligned}
& F\left(\lambda=\frac{i}{2}\right) \Phi(\{\lambda\})=\Lambda(\lambda,\{\lambda\}) \Phi(\{\lambda\}) \\
&=\left.\left[\left(\lambda+\frac{i}{2}\right)^{N} \prod_{k=1}^{l} f\left(\lambda-\lambda_{k}\right)+\left(\lambda-\frac{i}{2}\right)^{N} \prod_{k=1}^{l} h\left(\lambda-\lambda_{k}\right)\right]\right|_{\lambda=i / 2} \Phi(\{\lambda\}) \\
&=i^{N} \prod_{k=1}^{l} f\left(\frac{i}{2}-\lambda_{k}\right) \Phi(\{\lambda\}) \\
&=i^{N} \prod_{k=1}^{l} \frac{i / 2-\lambda_{k}-i}{i / 2-\lambda_{k}} \Phi(\{\lambda\}) \\
&=i^{N} \prod_{k=1}^{l} \frac{\lambda_{k}+i / 2}{\lambda_{k}-i / 2} \Phi(\{\lambda\})
\end{aligned}
$$


Hence,

$$
P \Phi=\frac{1}{i} \log \left(i^{-N} i^{N} \prod_{k=1}^{l} \frac{\lambda_{k}+i / 2}{\lambda_{k}-i / 2}\right) \Phi=\sum_{i=1}^{l} \frac{1}{i} \log \left(\frac{\lambda+i / 2}{\lambda-i / 2}\right) \Phi=\sum_{j=1}^{l} p\left(\lambda_{j}\right) \Phi
$$

where

$$
p(\lambda)=\frac{1}{i} \log \left(\frac{\lambda+i / 2}{\lambda-i / 2}\right)
$$

By operating $\Phi(\{\lambda\})$ with the expression for the Hamiltonian in (3.55), we get

$$
\begin{aligned}
H \Phi=\left(\left.\frac{i}{2} \frac{d}{d \lambda} \log F(\lambda)\right|_{\lambda=\frac{i}{2}}-\frac{N}{2}\right) \Phi=\left(\left.\frac{i}{2} \frac{F^{\prime}(\lambda)}{F(\lambda)}\right|_{\lambda=\frac{i}{2}}-\frac{N}{2}\right) \Phi \\
=\left[\frac { i } { 2 } \left(\frac{N\left(\lambda+\frac{i}{2}\right)^{N-1} \prod_{j=1}^{l} f\left(\lambda-\lambda_{j}\right)+\left(\lambda+\frac{i}{2}\right)^{N} \sum_{j=1}^{l} \prod_{k=1}^{l}\left(f\left(\lambda-\lambda_{j}\right)\right) \frac{i}{\left(\lambda-\lambda_{j}\right)^{2} f\left(\lambda-\lambda_{j}\right)}}{\left(\lambda+\frac{i}{2}\right)^{N} \prod_{j=1}^{l} f\left(\lambda-\lambda_{j}\right)+\left(\lambda-\frac{i}{2}\right)^{N} \prod_{j=1}^{l} h\left(\lambda-\lambda_{j}\right)}\right.\right. \\
\left.\left.+\frac{N\left(\lambda-\frac{i}{2}\right)^{N-1} \prod_{j=1}^{l} h\left(\lambda-\lambda_{j}\right)+\left(\lambda-\frac{i}{2}\right)^{N} \frac{d}{d \lambda} \prod_{j=1}^{l} h\left(\lambda-\lambda_{j}\right)}{\left(\lambda+\frac{i}{2}\right)^{N} \prod_{j=1}^{l} f\left(\lambda-\lambda_{j}\right)+\left(\lambda-\frac{i}{2}\right)^{N} \prod_{j=1}^{l} h\left(\lambda-\lambda_{j}\right)}\right)\left.\right|_{\lambda=\frac{i}{2}}-\frac{N}{2}\right] \Phi \\
=\left[\frac{i}{2}\left(\frac{N i^{N-1} \prod_{j=1}^{l} f\left(\frac{i}{2}-\lambda_{j}\right)+i^{N} \sum_{j=1}^{l} \prod_{j=1}^{l}\left(f\left(\frac{i}{2}-\lambda_{j}\right)\right) \frac{i}{\left(\frac{i}{2}-\lambda_{j}\right)^{2} f\left(\frac{i}{2}-\lambda_{j}\right)}}{i^{N} \prod_{j=1}^{l} f\left(\frac{i}{2}-\lambda_{j}\right)}-\frac{N}{2}\right] \Phi\right. \\
=\left[\frac{N}{2}-\frac{1}{2} \sum_{j=1}^{l} \frac{i}{\left(\lambda_{j}+\frac{i}{2}\right)\left(\lambda_{j}-\frac{i}{2}\right)}-\frac{N}{2}\right] \Phi \\
=-\frac{1}{2} \sum_{j=1}^{l} \frac{1}{\lambda_{j}^{2}+\frac{1}{4}} \Phi=\sum_{j} \epsilon\left(\lambda_{j}\right) \Phi
\end{aligned}
$$

where

$$
\epsilon(\lambda)=-\frac{1}{2} \frac{1}{\lambda^{2}+\frac{1}{4}}=\frac{1}{2} \frac{d}{d \lambda} p(\lambda)
$$


The fact that the Bethe vector state carries an energy and momentum allows us to treat it as a set of quasiparticles being formed in the spin chain. The parameter $\lambda$ is interpreted as the rapidity of the quasiparticle and the set of such parameters $\{\lambda\}$ uniquely determines the state. We can assert the latter because $\Lambda(\lambda,\{\lambda\})$ from equation (3.71) clearly becomes undefined when any two rapidities are equal. Hence, there cannot exist two rapidities $\lambda_{j}=\lambda_{k}$. As will be shown later on, considering only those rapidities for which $\lambda_{j} \neq \lambda_{k}$ will not cause any problems and the complete dimensionality of the system will still be restored.

Visualising the ground state of, say, the ferromagnetic spin chain model to be a series of up spins, flipping one of these spins leads to the creation of a quasiparticle, which is accomplished by acting with $B(\lambda)$ on the ground state. Hence, in a sense, the operator $B$ is the creation operator for the spin chain model, analogous to that of the harmonic oscillator model.

The quasiparticle so formed is also an eigenvector of the spin component $S^{3}$, and is found to have an eigenvalue of $\left(\frac{N}{2}-l\right)$, as in Appendix (A.4). That is,

$$
S^{3} \Omega=\left(\frac{N}{2}-l\right) \Omega
$$

This implies that the maximal spin state is the state with $l=0$, which is the highest weight state $\Omega$, with a spin of $\frac{N}{2}$. Moreover, the condition that the spin eigenvalue must be nonnegative means that $l \leq \frac{N}{2}$. Since $l$ is a whole number, the nature of the spin of the quasiparticles depends on whether $N$ is even or odd. In the former case, the spin eigenvalues are integers, corresponding to bosons, while an odd $N$ gives rises to half-integer spin eigenvalues, corresponding to fermions.

Considering the expressions for momentum and energy in equations (3.77) and (3.79), we see that

$$
\begin{gathered}
p(\lambda)=\frac{1}{i} \log \frac{\lambda+i / 2}{\lambda-i / 2}=\frac{1}{i} \log \frac{\frac{\lambda}{\sqrt{\lambda^{2}+1 / 4}}+\frac{1 / 2}{\sqrt{\lambda^{2}+1 / 4}} i}{\frac{\lambda}{\sqrt{\lambda^{2}+1 / 4}}-\frac{1 / 2}{\sqrt{\lambda^{2}+1 / 4}} i}=\frac{1}{i} \log \left(e^{2 i \theta}\right)=2 \theta=2 \cos ^{-1}\left(\frac{\lambda}{\sqrt{\lambda^{2}+1 / 4}}\right) \\
\Longrightarrow \cos \left(\frac{p}{2}\right)=\frac{\lambda}{\sqrt{\lambda^{2}+1 / 4}} \\
\Longrightarrow \cos p=\frac{\lambda^{2}-1 / 4}{\lambda^{2}+1 / 4}=1-\frac{1}{2} \frac{1}{\lambda^{2}+1 / 4}=1+\epsilon \\
\Longrightarrow \epsilon(\lambda)=\cos p(\lambda)-1
\end{gathered}
$$

Hence, for a positive hamiltonian such as $H$ defined above, the energy eigenvalues are always negative, in correspondence with the physical picture of the antiferromagnetic spin chain described 
by $H$. When the Hamiltonian is $-H$, the energy eigenvalues are positive, corresponding to a ferromagnetic spin chain.

\subsection{4 $\mathrm{XXX}_{1 / 2}$ model in the ferromagnetic thermodynamic limit $(N \rightarrow \infty)$}

As will be seen in this section, the thermodynamic limit of the Bethe Ansatz enables a formulation of the states as functions of continuous variables, thereby expressing their momenta and energies as integrals. To obtain these forms, we start by rewriting the BAE in a more usable form.

By taking the logarithm of the BAE (3.74) on both sides of the equation, we obtain

$$
\begin{gathered}
N \log \left(\frac{\lambda_{j}+i / 2}{\lambda_{j}-i / 2}\right)=\sum_{k=1}^{l} \log \left(\frac{\lambda_{j}-\lambda_{k}+i}{\lambda_{j}-\lambda_{k}-i}\right) \\
N i p(\lambda)=i \sum_{k=1}^{l} \phi\left(\lambda_{j}-\lambda_{k}\right)+2 \pi i Q_{j} \\
\Longrightarrow N p(\lambda)=2 \pi Q_{j}+\sum_{k=1}^{l} \phi\left(\lambda_{j}-\lambda_{k}\right)
\end{gathered}
$$

where $\phi\left(\lambda_{j}-\lambda_{k}\right)$ is a particular branch of $\log \left(\frac{\lambda+i}{\lambda-i}\right)$ and $\left\{Q_{j}: 0 \leq Q_{j} \leq N-1\right\}$ is an integer characterising the different branches and, thereby, characterising the rapidities themselves. Hence, $\left\{Q_{j}\right\}$, called the Bethe-Takahashi quantum numbers, have a one-to-one correspondence with the $\lambda_{j} \mathrm{~s}$.

Before exploring the boundaries on these quantum numbers, there are some results worth noting from the above equation. First of all, in the large $N$ and $Q_{j}$ limits, the second term goes to zero, since the rapidities tend to infinity. This leaves the relation

$$
p_{j}=2 \pi \frac{Q_{j}}{N}
$$

which is the quasicontinuous expression for the free particle momentum in a chain.

In the ferromagnetic case, this momentum relates to the energy by the relation $\epsilon(p)=1-\cos p$, since the Hamiltonian is $-H$.

Now, we take specific cases of $l$ values and substitute them in the general, to derive a general expression of energy and momentum for all values of $l$.

For $l=2$, we have two equations. 


$$
\begin{gathered}
\left(\frac{\lambda_{1}+i / 2}{\lambda_{1}-i / 2}\right)^{N}=\frac{\lambda_{1}-\lambda_{2}+i}{\lambda_{1}-\lambda_{2}-i}=e^{i \phi\left(\lambda_{1}-\lambda_{2}\right)} \Longrightarrow \frac{\lambda_{1}+i / 2}{\lambda_{1}-i / 2}=\xi_{1} e^{\frac{i}{N} \phi\left(\lambda_{1}-\lambda_{2}\right)} \\
\left(\frac{\lambda_{2}+i / 2}{\lambda_{2}-i / 2}\right)^{N}=\frac{\lambda_{2}-\lambda_{1}+i}{\lambda_{2}-\lambda_{1}-i}=e^{-i \phi\left(\lambda_{1}-\lambda_{2}\right)} \Longrightarrow \frac{\lambda_{2}+i / 2}{\lambda_{2}-i / 2}=\xi_{2} e^{-\frac{i}{N} \phi\left(\lambda_{1}-\lambda_{2}\right)}
\end{gathered}
$$

In the above equations, $\xi_{1}$ and $\xi_{2}$ are $N^{t h}$ roots of 1 and $\phi(\lambda)$ is the principal value of the function $\frac{\lambda-i}{\lambda+i}$, s argument, that is, $0 \leq \phi(\lambda) \leq 2 \pi$. Now, as $N \rightarrow \infty$, the exponentials in both equations tend to 1 and, in other words, the rapidities $\lambda_{1}$ and $\lambda_{2}$ are no longer dependent on each other and can independently be solved. Moreover, the Bethe vector they correspond to can be interpreted as the scattering of two independent particles, or 'magnons'.

Much more can be understood about these states by finding their energies and momenta. Say $\lambda_{1}=x_{1}+i y_{1}$ and $\lambda_{2}=x_{2}+i y_{2}$. Substituting into the first equation above, we get

$$
\left(\frac{x_{1}+i\left(y_{1}+1 / 2\right)}{x_{1}+i\left(y_{1}-1 / 2\right)}\right)^{N}=\frac{x_{1}-x_{2}+i\left(y_{1}-y_{2}+1\right)}{x_{1}-x_{2}+i\left(y_{1}-y_{2}-1\right)}
$$

Taking the moduli and squaring on both sides,

$$
\left(\frac{x_{1}^{2}+\left(y_{1}+1 / 2\right)^{2}}{x_{1}^{2}+\left(y_{1}-1 / 2\right)^{2}}\right)^{N}=\frac{\left(x_{1}-x_{2}\right)^{2}+\left(y_{1}-y_{2}+1\right)^{2}}{\left(x_{1}-x_{2}\right)^{2}+\left(y_{1}-y_{2}-1\right)^{2}}
$$

As $N \rightarrow \infty$, the LHS exponentially increases to $\infty$, provided that $y_{1}>0$. This requires that

$$
\left(x_{1}-x_{2}\right)^{2}+\left(y_{1}-y_{2}+1\right)^{2}=0 \Longrightarrow x_{1}=x_{2}, y_{1}-y_{2}=1
$$

Proceeding similarly for the second of the two Bethe equations, we get the same result, provided that $y_{2}<0$. Hence, without loss of generality, we can take $y_{1}>0$ and $y_{2}<0$.

Now, by multiplying both Bethe Ansatz Equations, we have

$$
\begin{gathered}
\left(\frac{\lambda_{1}+i / 2}{\lambda_{1}-i / 2}\right)^{N}\left(\frac{\lambda_{2}+i / 2}{\lambda_{2}-i / 2}\right)^{N}=1 \\
\Longrightarrow\left(\frac{x_{1}+i\left(y_{1}+1 / 2\right)}{x_{1}+i\left(y_{1}-1 / 2\right)} \cdot \frac{x_{2}+i\left(y_{2}+1 / 2\right)}{x_{2}+i\left(y_{2}-1 / 2\right)}\right)^{N}=1
\end{gathered}
$$

Using the results from equation (3.93), we get 


$$
\left(\frac{x_{1}+i\left(y_{1}+1 / 2\right)}{x_{1}+i\left(y_{1}-1 / 2\right)} \cdot \frac{x_{1}+i\left(y_{1}-1 / 2\right)}{x_{1}+i\left(y_{1}-3 / 2\right)}\right)^{N}=1 \Longrightarrow\left(\frac{x_{1}+i\left(y_{1}+1 / 2\right)}{x_{1}+i\left(y_{1}-3 / 2\right)}\right)^{N}=1
$$

which implies that $y_{1}=\frac{1}{2}$ and hence, $y_{2}=-\frac{1}{2}$. Rewriting $x_{1}=x_{2}$ as $\lambda_{1 / 2}$, we now have

$$
\lambda_{1}=\lambda_{1 / 2}+\frac{i}{2}, \lambda_{2}=\lambda_{1 / 2}-\frac{i}{2}
$$

where $\lambda_{1 / 2}$ is real. The momentum corresponding to $\lambda_{1 / 2}$ should be the same as $p\left(\lambda_{1}\right)+p\left(\lambda_{2}\right)$. That is,

$$
p_{1 / 2}(\lambda)=p_{0}\left(\lambda+\frac{i}{2}\right)+p_{0}\left(\lambda-\frac{i}{2}\right)=\frac{1}{i} \log \frac{\lambda_{1 / 2}+i / 2+i / 2}{\lambda_{1 / 2}+i / 2-i / 2} \cdot \frac{\lambda_{1 / 2}-i / 2+i / 2}{\lambda_{1 / 2}-i / 2-i / 2}=\frac{1}{i} \log \frac{\lambda_{1 / 2}+i}{\lambda_{1 / 2}-i}
$$

The corresponding energy

$$
\begin{gathered}
\epsilon_{1 / 2}(\lambda)=\frac{1}{2} \frac{d}{d \lambda} p_{1 / 2}(\lambda)=\frac{1}{2 i} \frac{d}{d \lambda} \log \frac{\lambda+i}{\lambda-i} \\
=\frac{1}{2 i} \frac{(\lambda-i)\{(\lambda-i)-(\lambda+i)\}}{(\lambda+i)(\lambda-i)^{2}} \\
\Longrightarrow \epsilon_{1 / 2}(\lambda)=\frac{1}{\lambda_{1 / 2}^{2}+1}=\frac{1}{2}\left(1-\cos p_{1 / 2}(\lambda)\right)
\end{gathered}
$$

If the momenta corresponding to $\lambda_{1}$ and $\lambda_{2}$ are $p_{1}$ and $p_{2}$ respectively and say the total momentum $p_{1}+p_{2}=p$, we see that for all possible momenta,

$$
\epsilon_{1 / 2}(p)<\epsilon_{0}\left(p_{1}\right)+\epsilon_{0}\left(p_{2}\right)
$$

where $\epsilon_{0}(p)$ is given by equation (3.84).

Through the above treatment, the fact that the energy $\epsilon_{1 / 2}(p)$ is lesser than the sum of energies of the individual magnons $\epsilon_{0}$ means that the state corresponding to $\lambda_{1 / 2}$ can be treated as the bound state of two individual magnons. Thus, $\lambda_{1}$ and $\lambda_{2}$ can be, in a sense, grouped into what is called a 'complex'.

By characterising complexes by the type $M\{M=0,1 / 2,1 \ldots\}$ where $(2 M+1)$ is the number of rapidities grouped into each of them, calling the above complex a type $1 / 2$ complex is justified. Faddev and Takhtajan also went on to show that for $l>2$, a generalisation of the above results could be made, and are as follows.

Each complex of type $M$ has rapidities of the form 


$$
\lambda_{M, m}=\lambda_{M}+i m ; m=-M,-M+1 \ldots M-1, M
$$

where $\lambda_{M}$ is a real number.

Since $m$ runs from $-M$ to $M$, the earlier claim that there are $(2 M+1)$ rapidities in a complex is justified and if we define $\nu_{M}$ to be the number of complexes of type $M$, it is apparent that

$$
l=\sum_{M} \nu_{M}(2 M+1)
$$

Hence, the problem of selecting a Bethe vector is altered to choosing a particular configuration of $\left\{\nu_{M}\right\}$.

The expression for the momentum of a type-M complex can be proved to be

$$
p_{M}(\lambda)=\frac{1}{i} \log \frac{\lambda+i(M+1 / 2)}{\lambda-i(M+1 / 2)}
$$

and the expression for the complex's energy is

$$
\epsilon_{M}(\lambda)=\frac{1}{2} \frac{2 M+1}{\lambda^{2}+(M+1 / 2)^{2}}=\frac{1}{2 M+1}\left(1-\cos p_{M}\right)
$$

The scattering matrix element for the scattering of a type- 0 complex with a type-M complex is given by

$$
S_{0, M}(\lambda)=\frac{\lambda+i M}{\lambda-i M} \cdot \frac{\lambda+i(M+1)}{\lambda-i(M+1)}
$$

and the scattering element for the scattering of a type-M complex with a type- $\mathrm{N}$ complex is given by

$$
S_{M, N}(\lambda)=\prod_{L=|M-N|}^{M+N} S_{0, M}(\lambda)
$$

All of the above results are derived in Appendix (A.5).

\subsubsection{BAE for an arbitrary configuration}

A Bethe vector is characterised by $\left\{\nu_{M}\right\}, q, l$, where $q=\sum_{M} \nu_{M}$ and $l=(2 M+1) \nu_{M}$. 
To understand the constraints on the Bethe-Takahashi quantum numbers and how they relate to the total number of vectors, we consider equation (3.74). To obtain the BAE for $\lambda_{M, j}$, the BAEs for each of the complexes need to be multiplied with each other. We thus get

$$
\begin{gathered}
e^{i p_{M}\left(\lambda_{M, j}\right) N}=\prod_{M^{\prime}} \prod_{\left(M^{\prime}, k\right) \neq(M, j)} S_{M, M^{\prime}}\left(\lambda_{M, j}-\lambda_{M^{\prime}, k}\right) \\
\Longrightarrow e^{i p_{M}\left(\lambda_{M, j}\right) N}=\prod_{M^{\prime}} \prod_{\left(M^{\prime}, k\right) \neq(M, j)} \prod_{L=\left|M-M^{\prime}\right|}^{M+M^{\prime}} S_{0, L}\left(\lambda_{M, j}-\lambda_{M^{\prime}, k}\right) \\
\Longrightarrow e^{i p_{M}\left(\lambda_{M, j}\right) N}=\prod_{M^{\prime}} \prod_{\left(M^{\prime}, k\right) \neq(M, j)} \prod_{L=\left|M-M^{\prime}\right|}^{M+M^{\prime}} \frac{\lambda_{M, j}-\lambda_{M^{\prime}, k}+i L}{\lambda_{M, j}-\lambda_{M^{\prime}, k}-i L} \cdot \frac{\lambda_{M, j}-\lambda_{M^{\prime}, k}+i(L+1)}{\lambda_{M, j}-\lambda_{M^{\prime}, k}-i(L+1)}
\end{gathered}
$$

In the above equations, we exclude the factor corresponding to $\left(M^{\prime}, k\right)=(M, j)$. Taking the logarithms on both sides, we get

$\left.N i p_{M}\left(\lambda_{M, j}\right)\right)=\sum_{M^{\prime}} \sum_{\left(M^{\prime}, k\right) \neq(M, j)} \sum_{L=\left|M-M^{\prime}\right|}^{M+M^{\prime}}\left(\log \frac{\lambda_{M, j}-\lambda_{M^{\prime}, k}+i L}{\lambda_{M, j}-\lambda_{M^{\prime}, k}-i L}+\log \frac{\lambda_{M, j}-\lambda_{M^{\prime}, k}+i(L+1)}{\lambda_{M, j}-\lambda_{M^{\prime}, k}-i(L+1)}\right)$

$$
\begin{aligned}
N i \frac{1}{i} \log \frac{\lambda_{M, j}+i(M+1 / 2)}{\lambda_{M, j}-i(M+1 / 2)}= & i \sum_{M^{\prime}} \sum_{\left(M^{\prime}, k\right) \neq(M, j)} \sum_{L=\left|M-M^{\prime}\right|}^{M+M^{\prime}} \frac{1}{i}\left(\log \frac{\lambda_{M, j}-\lambda_{M^{\prime}, k}+i L}{\lambda_{M, j}-\lambda_{M^{\prime}, k}-i L}+\right. \\
& \left.\log \frac{\lambda_{M, j}-\lambda_{M^{\prime}, k}+i(L+1)}{\lambda_{M, j}-\lambda_{M^{\prime}, k}-i(L+1)}\right)
\end{aligned}
$$

Using the branch

$$
\frac{1}{i} \log \frac{\lambda+i m}{\lambda-i m}=\pi-2 \tan ^{-1} \frac{\lambda}{m}
$$

we have 


$$
N\left(\pi-2 \tan ^{-1} \frac{\lambda_{M, j}}{M+1 / 2}\right)=\sum_{M^{\prime}} \sum_{\left(M^{\prime}, k\right) \neq(M, j)} \sum_{L=\left|M-M^{\prime}\right|}^{M+M^{\prime}}\left(2 \pi-\tan ^{-1}\left(\frac{\lambda_{M, j}-\lambda_{M^{\prime}, k}}{L}\right)-\tan ^{-1}\left(\frac{\lambda_{M, j}-\lambda_{M^{\prime}, k}}{L+1}\right)\right)
$$

$$
\begin{gathered}
\Longrightarrow N \pi-2 N \tan ^{-1} \frac{\lambda_{M, j}}{M+1 / 2}=2 \pi Q_{M, j}+\sum_{M^{\prime}} \sum_{\left(M^{\prime}, k\right) \neq(M, j)} \sum_{L=\left|M-M^{\prime}\right|}^{M+M^{\prime}} \\
\left\{-\tan ^{-1}\left(\frac{\lambda_{M, j}-\lambda_{M^{\prime}, k}}{L}\right)-\tan ^{-1}\left(\frac{\lambda_{M, j}-\lambda_{M^{\prime}, k}}{L+1}\right)\right\} \\
\Longrightarrow 2 N \tan ^{-1} \frac{\lambda_{M, j}}{M+1 / 2}=2 \pi Q_{M, j}+\sum_{M^{\prime}} \sum_{\left(M^{\prime}, k\right) \neq(M, j)} \Phi\left(\lambda_{M, j}-\lambda_{M^{\prime}, k}\right)
\end{gathered}
$$

where $Q_{M, j}$ is either an integer or a half-integer and

$$
\Phi(\lambda)=\sum_{L=\left|M-M^{\prime}\right|}^{M+M^{\prime}} 2\left(\tan ^{-1} \frac{\lambda}{L}+\tan ^{-1} \frac{\lambda}{L+1}\right)
$$

with $L \neq 0$.

The hypothesis of this analysis is that $Q_{M, j}$ uniquely determine the rapidities and increase monotonously with the corresponding rapidities. This also implies that, just as no two rapidities can coincide, $Q_{M, j} \neq Q_{M^{\prime}, k}$. The analysis is now continued by understanding the meaning of infinite rapidities.

Substituting $\lambda_{M, j}=\infty$ into the BAE, we see that infinite rapidities are also solutions of the Bethe equations. Since we hypothesised that $Q_{M, j}$ increases monotonously with $\lambda_{M, j}$, knowing the quantum number corresponding to an infinite rapidity allows us to determine the maximum quantum number for which the rapidity is finite.

Hence, we set $\lambda_{M, j}=\infty$ in equation (3.116) to get

$$
2 N \tan ^{-1}(\infty)=2 \pi Q_{M, \infty}+2 \sum_{M^{\prime}} \sum_{\left(M^{\prime}, k\right) \neq(M, j)} \sum_{L=\left|M-M^{\prime}\right|}^{M+M^{\prime}}\left(\tan ^{-1}(\infty)+\tan ^{-1}(\infty)\right)
$$


Since $\tan ^{-1}( \pm \infty)=\frac{\pi}{2}$, we have

$$
\begin{gathered}
N \pi=2 \pi Q_{M, \infty}+2 \sum_{M^{\prime}} \sum_{\left(M^{\prime}, k\right) \neq(M, j)} \sum_{L=\left|M-M^{\prime}\right|}^{M+M^{\prime}} \pi \Longrightarrow Q_{M, \infty}=\frac{N}{2}-\sum_{M^{\prime}} \sum_{\left(M^{\prime}, k\right) \neq(M, j)} \sum_{L=\left|M-M^{\prime}\right|}^{M+M^{\prime}} 1 \\
\Longrightarrow Q_{M, \infty}=\frac{N}{2}-\left\{\sum_{M^{\prime} \neq M} \sum_{\left(M^{\prime}, k\right) \neq(M, j)} \sum_{L=\left|M-M^{\prime}\right|}^{M+M^{\prime}} 1+\sum_{k \neq j}\left(\frac{1}{2}+\sum_{L=1}^{2 M} 1\right)\right\} \\
\Longrightarrow Q_{M, \infty}=\frac{N}{2}-\sum_{M^{\prime} \neq M}\left(2 \min \left(M, M^{\prime}\right)+1\right) \nu_{M^{\prime}}-\sum_{k \neq j}\left(\frac{1}{2}+2 M\right) \\
\Longrightarrow Q_{M, \infty}=\frac{N}{2}-\sum_{M^{\prime} \neq M}\left(2 \min \left(M, M^{\prime}\right)+1\right) \nu_{M^{\prime}}-\left(2 M+\frac{1}{2}\right)\left(\nu_{M}-1\right)
\end{gathered}
$$

We define $Q_{M, \max }$ to be the maximum quantum number for which the corresponding rapidity is finite. Therefore, for a quantum number higher than $Q_{M, \max }$, the corresponding rapidity is infinite. $Q_{M, \infty}$ corresponds to the case that all rapidities are infinite. Hence, since $Q_{M, j} \mathrm{~s}$ increase monotonously, we need to subtract the total number of roots $(2 M+1)$ from $Q_{M, \infty}$ to obtain $Q_{M, \max }$, that is,

$$
Q_{M, \max }=Q_{M, \infty}-(2 M+1)
$$

Using the above expression for $Q_{M, \infty}$, we can see that

$$
\begin{gathered}
Q_{M, \text { max }}=\frac{N}{2}-\sum_{M^{\prime} \neq M}\left(2 \min \left(M, M^{\prime}\right)+1\right) \nu_{M^{\prime}}-\left(2 M+\frac{1}{2}\right)\left(\nu_{M}-1\right)-(2 M+1) \\
\Longrightarrow Q_{M, \text { max }}=\frac{N}{2}-\sum_{M^{\prime} \neq M}\left(2 \min \left(M, M^{\prime}\right)+1\right) \nu_{M^{\prime}}-\left(2 M+\frac{1}{2}\right) \nu_{M}-\frac{1}{2} \\
\Longrightarrow Q_{M, \text { max }}=\frac{N}{2}-\sum_{M^{\prime}} J\left(M, M^{\prime}\right) \nu_{M^{\prime}}-\frac{1}{2}
\end{gathered}
$$

where

$$
J\left(M, M^{\prime}\right)= \begin{cases}\left(2 \min \left(M, M^{\prime}\right)+1\right) & M^{\prime} \neq M \\ \left(2 M+\frac{1}{2}\right) & M^{\prime}=M\end{cases}
$$


Since $\tan ^{-1}(\lambda)$ is an odd function and $Q_{M, j}$ varies monotonously with $\lambda_{M, j}$,

$$
Q_{M, \min }=-Q_{M, \max }
$$

Hence, the number of quantum numbers, or 'vacances', for a complex of type $M$ is

$$
P_{M}=Q_{M, \max }-Q_{M, \min }+1=2 Q_{M, \max }+1=N-2 \sum_{M^{\prime}} J\left(M, M^{\prime}\right) \nu_{M^{\prime}}
$$

\subsubsection{Dimensionality of the Spin Chain with the Complex Hypothesis}

In order for the above hypotheses to hold, we need to prove that the dimensionality of the problem still holds viz. the total number of Bethe vectors summed over all $\left\{l, q,\left\{\nu_{M}\right\}\right\}$ is equal to $2^{N}$.

To do this, we first note that for a particular $N,\left\{\nu_{M}\right\}$, the number of ways in which $P_{M}$ vacances can be distributed over $\left\{\nu_{M}\right\}$ is given by

$$
Z\left(N,\left\{\nu_{M}\right\}\right)=\prod_{M}\left(\begin{array}{l}
P_{M} \\
\nu_{M}
\end{array}\right)
$$

We need to sum the above quantity over all permutations $\left\{\nu_{M}\right\}$ that satisfy $l=\sum_{M}(2 M+1) \nu_{M}$ and $q=\sum_{M} \nu_{M}$ for a given 1 and $\mathrm{q}$.

$$
Z(N, l, q)=\sum_{\sum_{M}(2 M+1) \nu_{M}=l} Z\left(N,\left\{\sum_{M} \nu_{M}\right\}\right)
$$

We can calculate the above quantity by replacing $\left\{\nu_{M}\right\}$ with $\left\{\nu_{M}\right\}$ such that

$$
\nu_{M}^{\prime}=\nu_{M+1 / 2}, \quad M=0, \frac{1}{2}, 1 \ldots
$$

This implies that 


$$
\begin{array}{rl}
P_{M}\left(N,\left\{\nu_{M}\right\}\right)=N & -2 J(M, 0) \nu_{0}-2 \sum_{M^{\prime} \geq 1 / 2} J\left(M, M^{\prime}\right) \nu_{M}^{\prime} \\
= & N-2 \nu_{0}-2 \sum_{M^{\prime} \geq 1 / 2}\left(J\left(M-\frac{1}{2}, M^{\prime}-\frac{1}{2}\right)+1\right) \nu_{M}^{\prime} \\
=N & 2 \nu_{0}-2 \sum_{M^{\prime} \geq 1 / 2} \nu_{M}^{\prime}-2 \sum_{M^{\prime} \geq 1 / 2}\left(J\left(M-\frac{1}{2}, M^{\prime}-\frac{1}{2}\right)\right) \nu_{M}^{\prime} \\
& =N-2 q-2 \sum_{M^{\prime} \geq 1 / 2}\left(J\left(M-\frac{1}{2}, M^{\prime}-\frac{1}{2}\right)\right) \nu_{M}^{\prime} \\
= & N-2 q-2 \sum_{M^{\prime}}\left(J\left(M-\frac{1}{2}, M^{\prime}\right)\right) \nu_{M}^{\prime}=P_{M-1 / 2}\left(N-2 q,\left\{\nu_{M}^{\prime}\right\}\right)
\end{array}
$$

In the above derivation, we used the fact that $J\left(M, M^{\prime}\right)=J\left(M-\frac{1}{2}, M^{\prime}-\frac{1}{2}\right)+1$, which is true because

$$
\begin{gathered}
J\left(M-\frac{1}{2}, M^{\prime}-\frac{1}{2}\right)+1= \begin{cases}\left.2 \min \left(M-\frac{1}{2}, M^{\prime}-\frac{1}{2}\right)\right)+1+1 & M^{\prime} \neq M \\
2\left(M-\frac{1}{2}\right)+\frac{1}{2}+1 & M^{\prime}=M\end{cases} \\
\Longrightarrow J\left(M-\frac{1}{2}, M^{\prime}-\frac{1}{2}\right)+1=\left\{\begin{array}{ll}
2 \min \left(M, M^{\prime}\right)+1 & M^{\prime} \neq M \\
\left.2 M+\frac{1}{2}\right) & M^{\prime}=M
\end{array}=J\left(M, M^{\prime}\right)\right.
\end{gathered}
$$

We now have

$$
Z\left(N,\left\{\nu_{M}\right\}\right)=\left(\begin{array}{c}
P_{0} \\
\nu_{0}
\end{array}\right) Z\left(N-2 q,\left\{\nu_{M}^{\prime}\right\}\right)
$$

Summing over all allowed values of $\nu_{0}=\nu$,

$$
Z(N, l, q)=\prod_{\nu=0}^{q-1}\left(\begin{array}{c}
N-2 q+\nu \\
\nu
\end{array}\right) Z(N-2 q, l-q, q-\nu)
$$

By induction, it can be shown that

$$
Z(N, l, q)=\frac{N-2 l+1}{N-l+1}\left(\begin{array}{c}
N-l+1 \\
q
\end{array}\right)\left(\begin{array}{l}
l-1 \\
q-1
\end{array}\right)
$$

as is done in Appendix (A.6-(i)). 
Now,

$Z(N, l)=\sum_{q=1}^{l} Z(N, l, q)=\frac{N-2 l+1}{N-l+1} \sum_{q=1}^{l}\left(\begin{array}{c}N-l+1 \\ q\end{array}\right)\left(\begin{array}{l}l-1 \\ q-1\end{array}\right)=\frac{N-2 l+1}{N-l+1} \sum_{q=0}^{l}\left(\begin{array}{c}N-l+1 \\ q\end{array}\right)\left(\begin{array}{c}l-1 \\ l-q\end{array}\right)$

Using the fact that $\sum_{r=0}^{k}\left(\begin{array}{c}n-m \\ r\end{array}\right)\left(\begin{array}{c}m \\ k-r\end{array}\right)=\left(\begin{array}{l}n \\ k\end{array}\right)$, we have

$$
\begin{aligned}
Z(N, l)=\sum_{q=1}^{l} Z(N, l, q)=\frac{N-2 l+1}{N-l+1}\left(\begin{array}{c}
N \\
l
\end{array}\right) & \\
& =\left(1-\frac{l}{N-l+1}\right)\left(\begin{array}{c}
N \\
l
\end{array}\right)=\left(\begin{array}{c}
N \\
l
\end{array}\right)-\left(\begin{array}{c}
N \\
l-1
\end{array}\right)
\end{aligned}
$$

Now, since a Bethe vector of spin $\frac{N}{2}-l$ is the highest weight of a spin multiplet of dimension $2\left(\frac{N}{2}-l\right)+1=(N-2 l+1)$ states, we can show that

$$
Z=\sum_{l}(N-2 l+1) Z(N, l)=2^{N}
$$

as is done in Appendix (A.6-(ii))

Hence, we find that we do in fact count $2^{N}$ Bethe vectors using the hypothesis about the quantum numbers $\left\{Q_{M, j}\right\}$, which is the dimension of the Hilbert space corresponding to the model. As a result, the hypothesis of rapidities being grouped into complexes which correspond to bound states is further strengthened as the system it describes is complete.

\subsection{7 $\mathrm{XXX}_{1 / 2}$ model in the antiferromagnetic thermodynamic limit}

The next goal is to classify the ground states and the first few excited states in the thermodynamic limit. In order to do this, we consider the antiferromagnetic spin chain model.

The ground state for the antiferromagnetic case corresponds to $\nu_{0}=\frac{N}{2} ; \nu_{M}=0, M \geq \frac{1}{2}$, which is the state of lowest energy and also has a maximal number of real roots.

For this state, $l=q=\frac{N}{2} \Longrightarrow S^{3}=\frac{N}{2}-l=\frac{N}{2}-\frac{N}{2}=0$

The number of vacances is non-zero only for $M=0$.

$P_{0}=N-2 J(0,0) \nu_{0}=N-2 \cdot \frac{1}{2} \cdot \frac{N}{2}=N-\frac{N}{2}=\frac{N}{2}$ 
Thus, the number of vacances $P_{0}$ is equal to the number of roots $q$, which means that there is no freedom in distributing the $\frac{N}{2}$ quantum numbers $Q_{0, j}$ and they span the whole interval

$$
-\frac{N}{4}+\frac{1}{2} \leq Q_{0, j} \leq \frac{N}{4}-\frac{1}{2}
$$

Under the assumption that $N$ is even, the quantum numbers are integers when $\frac{N}{2}$ is odd and half-integers when $\frac{N}{2}$ is even.

Therefore, in the ground state, there is no room for excitations and this state is the Dirac sea of quasiparticles. Due to the condition that all quantum numbers must be distinct, this state is unique

The state with next lowest energy must have $\nu_{0}=\frac{N}{2}-1 ; \nu_{M}=0, M \geq 1 / 2$

$\Longrightarrow l=q=\frac{N}{2}-1$.

The number of vacances is again non-zero only for $M=0$.

$P_{0}=N-2 \cdot \frac{1}{2} \cdot\left(\frac{N}{2}-1\right)=\frac{N}{2}+1$

In this case, the number of vacances exceeds the number of roots $q$ by two. This means that two admissible quantum numbers cannot be used, giving rise to a degeneracy in the state or 'holes'.

We can continue by considering states with $\nu_{0}=\frac{N}{2}-\kappa$, which all have higher energy than the ground state and correspond to a series of excited states.

The next lowest states correspond to $\nu_{0}=\frac{N}{2}-2$. In this case, there are two possibilities that still ensure that $l \leq \frac{N}{2}$, which is the governing rule.

(i) $\nu_{0}=\frac{N}{2}-2 ; \nu_{1 / 2}=1 ; \quad \nu_{M}=0, M \geq 1 \Longrightarrow l=\frac{N}{2} ; S^{3}=0$

$$
\begin{gathered}
P_{0}=N-2 J(0,0) \nu_{0}-2 J(0,1 / 2) \nu_{1 / 2}=N-\frac{N}{2}+2-2=\frac{N}{2} \\
P_{1 / 2}=N-2 J(1 / 2,0) \nu_{0}-2 J(1 / 2,1 / 2) \nu_{1 / 2}=N-2\left(\frac{N}{2}-2\right)-2\left(\frac{3}{2}\right)=1
\end{gathered}
$$

In this case, the total number of vacances exceeds the number of roots by two, and for the type- $1 / 2$ complex, there is only one vacance corresponding to one root, meaning that any freedom is due to the type-0 complex.

(ii) $\nu_{0}=\frac{N}{2}-2 ; \quad \nu_{M}=0, M \geq \frac{1}{2} \Longrightarrow l=\frac{N}{2}-2 ; S^{3}=2$ 


$$
P_{0}=N-2 J(0,0) \nu_{0}=\frac{N}{2}+2
$$

In this case, the number of vacances exceeds the number of roots by four.

We can extrapolate this to see that for $\nu_{0}=\frac{N}{2}-\kappa ; \nu_{M}=0, M \geq 1 / 2$

$$
P_{0}=\frac{N}{2}+\kappa ; q=\frac{N}{2}-\kappa
$$

Hence, the number of vacances exceeds the number of roots by $2 \kappa$ or in other words, there are $2 \kappa$ holes, while the spin of the state is $\kappa$.

The above treatment of the antiferromagnetic states is purely discrete and does not take into account the thermodynamic limit. When $N \rightarrow \infty$, the roots become quasicontinuous and summations corresponding to energy and momentum are replaced by integrals, which allow us to understand the physical spectrum more precisely.

(i) Ground state

We first consider the ground state where, as we have seen, there are no holes and the interval is completely spanned. Since the hypothesis that the quantum numbers vary montonously is true, we can safely make the claim that

$$
Q_{0, j}=j
$$

for $\mathrm{N} / 2$ odd, in which case the quantum numbers are integers. The Bethe Ansatz equations written in the form of equation (3.116) now become

$$
\begin{aligned}
& 2 N \tan ^{-1}\left(2 \lambda_{j}\right)=2 \pi j+\sum_{k} \tan ^{-1}\left(\lambda_{j}-\lambda_{k}\right) \\
& \Longrightarrow \tan ^{-1}\left(2 \lambda_{j}\right)=\frac{\pi j}{N}+\frac{1}{N} \sum_{k} \tan ^{-1}\left(\lambda_{j}-\lambda_{k}\right)
\end{aligned}
$$

Say $x=\frac{j}{N}$. In the limit $N \rightarrow \infty, x$ becomes a continuous variable. Furthermore,

$$
\begin{gathered}
-\frac{N}{4}+\frac{1}{2} \leq Q_{0, j} \leq \frac{N}{4}-\frac{1}{2} \\
\Longrightarrow-\frac{1}{4}+\frac{1}{2 N} \leq \frac{j}{N} \leq \frac{1}{4}-\frac{1}{2 N}
\end{gathered}
$$




$$
N \rightarrow \infty \Longrightarrow-\frac{1}{4} \leq x \leq \frac{1}{4}
$$

In the thermodynamic limit, the roots $\lambda_{j}$ become functions $\lambda(x)$ and the above form of the BAE becomes

$$
\tan ^{-1}(2 \lambda(x))=\pi x+\int_{-1 / 4}^{1 / 4} \tan ^{-1}(\lambda(x)-\lambda(y)) d y
$$

While the form of $\lambda x$ isn't necessary or important for the forthcoming analysis, expressions for the energy eigenvalues $h\left(\lambda_{j}\right)$ and momentum eigenvalues $p\left(\lambda_{j}\right)$ are extremely essential to derive. In the thermodynamic limit, we have

$$
\sum_{j} h\left(\lambda_{j}\right)=N \int_{-1 / 4}^{1 / 4} h(\lambda(x)) d x=N \int_{-\infty}^{\infty} h(\lambda) \rho(\lambda) d \lambda
$$

where $\rho(\lambda)$ is a density function, in a sense, defined by

$$
\rho(\lambda)=\frac{d x}{d \lambda}=\frac{1}{\lambda^{\prime}(x)}
$$

Hence, by determining the density, we can obtain expressions for both energy and momentum.

We start by differentiating equation (3.153) with respect to $\lambda$ to get

$$
\frac{2}{1+4 \lambda^{2}}=\pi \rho_{0}(\lambda)+\int_{-\infty}^{\infty} \frac{\rho_{0}(\mu)}{1+(\lambda-\mu)^{2}} d \mu
$$

where the subscript in $\rho_{0}(\lambda)$ signifies that it is the ground state density.

This integral can be solved by Fourier transform, as is done in Appendix (A.7-(i)), to get

$$
\rho_{0}(\lambda)=\frac{1}{2 \cosh (\pi \lambda)}
$$

We can use the density function to calculate the energy and the momentum of the ground state.

The momentum is given by 


$$
P_{0}=N \int_{-\infty}^{\infty} p_{0}(\lambda) \rho_{0}(\lambda) d \lambda=0
$$

since $p_{0}=-2 \tan ^{-1}(2 \lambda)$, obtained by taking a different branch of the logarithm, is an odd function and density function is an even function, thereby making the product an odd function.

The energy of the ground state is

$$
E_{0}=N \int_{-\infty}^{\infty} \epsilon_{0}(\lambda) \rho_{0}(\lambda) d \lambda=-N \log 2
$$

which is derived in Appendix (A.7-(ii)).

(ii) Triplet state

In this case, as we have seen, $\nu_{0}=\frac{N}{2}-1 ; \nu_{M}=0, M \geq 1 / 2$, and there are two holes at, say, $j_{1}$ and $j_{2}$.

The holes do not correspond to any of the roots and should therefore be omitted in the expression for $Q_{0, j}$. The omission can be done by using a step function

$$
\theta(x)= \begin{cases}1 & x \geq 0 \\ 0 & x \leq 0\end{cases}
$$

Hence, we have

$$
Q_{0, j}=j+\theta\left(j-j_{1}\right)+\theta\left(j-j_{2}\right)
$$

The spin of this state was seen to be 1 , meaning that it corresponds to the $(2 S+1)=3$ or triplet state.

Performing the same set of operations on the BAE as was done for the ground state,

$$
\begin{aligned}
& 2 N \tan ^{-1}\left(2 \lambda_{j}\right)=2 \pi\left(j+\theta\left(j-j_{1}\right)+\theta\left(j-j_{2}\right)\right)+2 \sum_{k} \tan ^{-1}\left(\lambda_{j}-\lambda_{k}\right) \\
& \Longrightarrow \tan ^{-1}\left(2 \lambda_{j}\right)=\frac{1}{N} \pi\left(j+\theta\left(j-j_{1}\right)+\theta\left(j-j_{2}\right)\right)+\frac{1}{N} \sum_{k} \tan ^{-1}\left(\lambda_{j}-\lambda_{k}\right)
\end{aligned}
$$

In the thermodynamic limit, this equation becomes 


$$
\Longrightarrow \tan ^{-1}(2 \lambda(x))=\pi x+\frac{\pi}{N}\left(\theta\left(j-j_{1}\right)+\theta\left(j-j_{2}\right)\right)+\frac{1}{N} \int_{-1 / 4}^{1 / 4} \tan ^{-1}(\lambda(x)-\lambda(y)) d y
$$

Differentiating, we get

$$
\Longrightarrow \frac{2}{1+4 \lambda^{2}}=\pi \rho_{t}(\lambda)+\int_{-\infty}^{\infty} \frac{\rho_{t}(\mu)}{1+(\lambda-\mu)^{2}} d \lambda+\frac{\pi}{N}\left(\delta\left(\lambda-\lambda_{1}\right)+\delta\left(\lambda-\lambda_{2}\right)\right)
$$

Using equation (3.153), we have

$$
\rho_{t}(\lambda)=\rho_{0}(\lambda)+\frac{1}{N}\left(\sigma\left(\lambda-\lambda_{1}\right)+\sigma\left(\lambda-\lambda_{2}\right)\right)
$$

where

$$
\sigma(\lambda)+\frac{1}{\pi} \int_{-\infty}^{\infty} \frac{\sigma(\mu))}{1+(\lambda-\mu)^{2}} d \mu+\delta(\lambda)=0
$$

The form of $\sigma(\lambda)$ is found to be

$$
\sigma(\lambda)=\frac{1}{2 \pi} \int_{-\infty}^{\infty} \hat{\sigma}(\xi) e^{-i \lambda \xi} d \xi ; \hat{\sigma}(\xi)=-\frac{1}{1+e^{-|\xi|}}
$$

which is verified in Appendix (A.7-(iii)).

We measure the energy and momentum from the ground state. Hence,

$$
\epsilon_{t}\left(\lambda_{1}, \lambda_{2}\right)=N \int_{-\infty}^{\infty} h(\lambda)\left(\rho_{t}(\lambda)-\rho_{0}(\lambda)\right) d \lambda=\epsilon\left(\lambda_{1}\right)+\epsilon\left(\lambda_{2}\right)
$$

where

$$
\epsilon(\lambda)=\int_{-\infty}^{\infty} h(\lambda) \sigma(\lambda-\mu) d \mu=\frac{\pi}{2 \cosh (\pi \lambda)}
$$

as derived in Appendix (A.7-(iv)).

We also have

$$
p_{t}\left(\lambda_{1}, \lambda_{2}\right)=N \int_{-\infty}^{\infty} p(\lambda)\left(\rho_{t}(\lambda)-\rho_{0}(\lambda)\right) d \lambda=p\left(\lambda_{1}\right)+p\left(\lambda_{2}\right)
$$


where

$$
p_{t}(\lambda)=\int_{-\infty}^{\infty} p(\mu) \sigma(\lambda-\mu) d \mu=\tan ^{-1}(\sinh (\pi \lambda))
$$

as derived in Appendix (A.7-(v)).

The energy can also be written in terms of the momentum as

$$
\epsilon(k)=\frac{\pi}{2} \cos k, \quad-\frac{\pi}{2} \leq k \leq \frac{\pi}{2}
$$

which holds because

$$
\frac{\pi}{2} \cos k=\frac{\pi}{2} \cos \left(\tan ^{-1}(\sinh (\pi \lambda))\right)=\frac{\pi}{2} \frac{1}{\sqrt{1+\sinh ^{2}(\pi \lambda)}}=\frac{\pi}{2 \cosh (\pi \lambda)}
$$

\section{(iii) Singlet state}

For this state, $\nu_{0}=\frac{N}{2}-2 ; \nu_{1 / 2}=1 ; \nu_{M}=0, M \geq 1 / 2$, and has two holes. The net spin of the state is zero, meaning that it is a singlet state. Since there are now multiple types of complexes, we have multiple equations.

$$
\frac{2}{1+4 \lambda^{2}}=\pi \rho_{s}(\lambda)+\int_{-\infty}^{\infty} \frac{\rho_{s}(\mu)}{1+(\lambda-\mu)^{2}} d \mu+\frac{1}{N}\left(\delta\left(\lambda-\lambda_{1}\right)+\delta\left(\lambda-\lambda_{2}\right)+\Phi_{0,1 / 2}^{\prime}\left(\lambda-\lambda_{1 / 2}\right)\right)
$$

Corresponding to $\lambda_{1 / 2}$, we have the following equation. Since $P_{1 / 2}=1$, there is only admissible value of $Q_{1 / 2, j}$ which has to be zero due to symmetry.

$$
\tan ^{-1} \lambda_{1 / 2}=\frac{1}{N} \sum_{j} \Phi_{1 / 2,0}\left(\lambda_{1 / 2}-\lambda_{0, j}\right)
$$

The first of the two equations gives

$$
\rho_{s}(\lambda)=\rho_{0}(\lambda)+\frac{1}{N}\left(\sigma\left(\lambda-\lambda_{1}\right)+\sigma\left(\lambda-\lambda_{2}\right)+\omega\left(\lambda-\lambda_{1 / 2}\right)\right)
$$

where $\sigma(\lambda)$ is as previously defined and $\omega(\lambda)$ is defined such that

$$
\pi \omega(\lambda)+\int_{-\infty}^{\infty} \frac{\omega(\mu)}{1+(\lambda-\mu)^{2}} d \mu+\Phi_{0,1 / 2}^{\prime}(\lambda)=0
$$


It can be shown that

$$
\omega(\lambda)=-\frac{2}{\pi\left(1+4 \lambda^{2}\right)}
$$

as is done in Appendix (A.7-(vi)).

We can rewrite equation (3.173) as the following.

$$
\tan ^{-1} \lambda_{1 / 2}-\int_{-\infty}^{\infty} \Phi_{1 / 2,0}\left(\lambda_{1 / 2}-\lambda\right) \rho_{0}(\lambda) d \lambda=\frac{1}{N} \int_{-\infty}^{\infty} \Phi_{1 / 2,0}\left(\lambda_{1 / 2}-\lambda\right)\left\{\sigma\left(\lambda-\lambda_{1}\right)+\sigma\left(\lambda-\lambda_{2}\right)+\omega\left(\lambda-\lambda_{1 / 2}\right)\right\} d \lambda
$$

The left hand side of the equation vanishes for every value of $\lambda_{1 / 2}$. The oddness of $\Phi$ and the evenness of $\omega$ means that the integral of the third term on the right hand side also vanishes. This leaves

$$
\begin{gathered}
\int_{-\infty}^{\infty} \Phi_{1 / 2,0}\left(\lambda_{1 / 2}-\lambda\right)\left\{\sigma\left(\lambda-\lambda_{1}\right)+\sigma\left(\lambda-\lambda_{2}\right)\right\} d \lambda=0 \\
\Longrightarrow \tan ^{-1}\left(2\left(\lambda_{1 / 2}-\lambda_{1}\right)\right)+\tan ^{-1}\left(2\left(\lambda_{1 / 2}-\lambda_{2}\right)\right)=0 \\
\Longrightarrow \lambda_{1 / 2}=\frac{\lambda_{1}+\lambda_{2}}{2}
\end{gathered}
$$

Now, the energy and momentum contributions of the $\omega$ term interestingly cancel out the corresponding contributions of the type-1/2 complex, as is shown in Appendices (A.7-(vii) and (A.7-(viii)). That is,

$$
\int_{-\infty}^{\infty} h(\lambda) \omega\left(\lambda-\lambda_{1 / 2}\right) d \lambda=-h_{1 / 2}\left(\lambda_{1 / 2}\right)
$$

and

$$
\int_{-\infty}^{\infty} p(\lambda) \omega\left(\lambda-\lambda_{1 / 2}\right) d \lambda=-p_{1 / 2}\left(\lambda_{1 / 2}\right)
$$

This means that the energy and momentum of the state are only the contributions of the individual magnons. 


$$
\begin{aligned}
& \epsilon_{s}\left(\lambda_{1}, \lambda_{2}\right)=h_{1 / 2}\left(\lambda_{1 / 2}\right)+\int_{-\infty}^{\infty} h(\lambda)\left(\rho_{s}(\lambda)-\rho(\lambda)\right) d \lambda=\epsilon\left(\lambda_{1}\right)+\epsilon\left(\lambda_{2}\right) \\
& k_{s}\left(\lambda_{1}, \lambda_{2}\right)=k_{1 / 2}\left(\lambda_{1 / 2}\right)+\int_{-\infty}^{\infty} k(\lambda)\left(\rho_{s}(\lambda)-\rho(\lambda)\right) d \lambda=k\left(\lambda_{1}\right)+k\left(\lambda_{2}\right)
\end{aligned}
$$

Hence, the energy of the singlet state is the same as that of the triplet state. The only difference is in the spin. The singlet state has no net spin. For higher energy states, it was proved that the same holds. That is, the energy and momentum depend only on the number of particles and the contributions of any complexes vanish. 


\section{Chapter 4}

\section{Scattering and Interaction of Magnons}

\subsection{Yang-Baxter Equations through Consistency Conditions}

We consider the situation of two particles colliding with each other in a one-dimensional space. When a particle with momentum $k_{1}$ scatters with another particle with momentum $k_{2}$, the probabilities of it reflecting, or bouncing off the second particle, and transmitting, or passing through the second particle, are directly related to the reflection amplitude $R\left(k_{1}, k_{2}\right)$ and transmission amplitude $T\left(k_{1}, k_{2}\right)$, respectively. We consider the scenario that the momenta are either retained or exchanged. When we assume that the system has Galilean invariance, these amplitudes only depend on the difference between the momenta. Since there is no possibility of anything apart from reflection and transmission occurring, the sum of the squares of these amplitudes must be 1 .

The particles can either retain or exchange their momenta post-scattering. The momenta can be directly linked to quantum numbers, say, $n_{1}$ and $n_{2}$ before and $n_{1}^{\prime}$ and $n_{2}^{\prime}$ after collision. If the particles exchange quantum numbers, that is, $n_{2}^{\prime}=n_{1}$ and $n_{1}^{\prime}=n_{2}$

The collection of all amplitudes is the S-matrix, which is unitary since only reflection or transmission can occur.

When we consider three-particles scattering in one dimension, we label the wavefunction of a permutation of the particles by $\Psi(P)$, where $P$ is a particular permutation of the momenta with respect to the particles. Hence, for example, $\Psi(123)$ is the amplitude corresponding to particle 1,2 , and 3 having momenta $k_{1}, k_{2}$, and $k_{3}$ respectively. 
Let the permutation operators $\alpha_{1}$ permute the first two momenta in $P$ and $\alpha_{2}$ permute the last two momenta in $P$. We see that, from any initial state, we can get to the same final state through multiple combinations of the permutation operators. This gives rise to the following conditions for consistency.

The first condition is trivial to see, based on the fact that permuting the same two momenta twice will return to the original configuration.

$$
\alpha_{1}^{2}=\alpha_{2}^{2}=I
$$

Next, we see that if we want to get from the permutation (123) to the permutation (321), this can be accomplished by two different combinations of three terms, giving the following.

$$
\alpha_{1} \alpha_{2} \alpha_{1}=\alpha_{2} \alpha_{1} \alpha_{2}
$$

Now, we define the S-matrix as the matrix giving the relation between the amplitudes of any two wavefunctions. For example,

$$
\Psi(213)=S_{1}\left(k_{1}, k_{2}\right) \Psi(123)
$$

Using the representation that the diagonal elements of the S-matrix are the reflection amplitudes while the off-diagonal elements are the transmission amplitudes, we have

$$
S_{i}=R_{j k}+T_{j k} \alpha_{i}
$$

The above relation implies that the S-matrices obey a relation similar to equation (4.2).

$$
S_{1}\left(k_{1}, k_{3}\right) S_{2}\left(k_{1}, k_{3}\right) S_{1}\left(k_{1}, k_{2}\right)=S_{2}\left(k_{1}, k_{2}\right) S_{1}\left(k_{1}, k_{3}\right) S_{2}\left(k_{2}, k_{3}\right) \equiv S_{1} S_{2} S_{1}=S_{2} S_{1} S_{2}
$$

which is called the Yang-Baxter equation. This is essentially a consistency condition that must be obeyed for the scattering to be diffractionless. However, there is something much more fundamental that can be said about a system whose scattering operators satisfy the Yang-Baxter equation as will now be shown.

When the number of particles is increased, the consistency conditions are similar in form. We have 


$$
S_{i}^{2}=1 \Longrightarrow S_{i} S_{j}=I \quad(i=j)
$$

which arises from the fact that the S-matrix is unitary.

For two scatterings which don't involve the same particle, or are far away from each other, we have the following, since neither scattering affects the other.

$$
S_{i} S_{j}=S_{j} S_{i} \Longrightarrow\left(S_{i} S_{j}\right)^{2}=I(|i-j|>1)
$$

where the second form of the relation is obtained by using the previous relation.

For two scatterings with one particles involved in both, we have the Yang-Baxter equation described above.

$$
S_{i} S_{j} S_{i}=S_{j} S_{i} S_{j} \Longrightarrow\left(S_{i} S_{j}\right)^{3}=I \quad(|i-j|=1)
$$

Hence, we see that any scattering process can be equivalently described by a series of two-body scatterings.

When a scattering process occurs, the particles also undergo a phase shift specified by a function $\theta(k)$, where $k$ is the relative momentum between the two scattering particles. The phase shift transforms the wavefunction $\Psi \rightarrow e^{-i \theta(k)} \Psi$. We define $\Theta(k)=-e^{-i \theta(k)}$.

If we write the three-particle S-matrix explicitly in matrix form with the transmission coefficients along the diagonal (these representations are interchangeable), we can factor out the phase factor by defining a reduced reflection amplitude $r_{i j}=R\left(k_{i}-k_{j}\right) / \Theta$ and a reduced transmission amplitude $t_{i j}=T\left(k_{i}-k_{j}\right) / \Theta$ as follows.

$$
S\left(k_{2}, k_{3}\right)=\left(\begin{array}{ccc}
\Theta(k) & 0 & 0 \\
0 & T(k) & R(k) \\
0 & R(k) & T(k)
\end{array}\right)=\Theta\left(k_{2}-k_{3}\right)\left(\begin{array}{ccc}
1 & 0 & 0 \\
0 & T_{23} & R_{23} \\
0 & R_{23} & T_{23}
\end{array}\right)
$$

Similarly,

$$
S\left(k_{1}, k_{3}\right)=\Theta\left(k_{1}-k_{3}\right)\left(\begin{array}{ccc}
T_{13} & 0 & R_{13} \\
0 & 1 & 0 \\
R_{13} & 0 & T_{13}
\end{array}\right)
$$




$$
S\left(k_{1}, k_{2}\right)=\Theta\left(k_{1}-k_{2}\right)\left(\begin{array}{ccc}
T_{12} & R_{12} & 0 \\
R_{12} & T_{12} & 0 \\
0 & 0 & 1
\end{array}\right)
$$

We take this a step further and substitute these forms of the matrices in the Yang-Baxter equation (4.5) to obtain a general relation for the reflection and transmission amplitudes of an integrable system. Since the products of phase shifts cancel out, we are left with

$$
\begin{gathered}
\left(\begin{array}{ccc}
1 & 0 & 0 \\
0 & t_{23} & r_{23} \\
0 & r_{23} & t_{23}
\end{array}\right)\left(\begin{array}{ccc}
t_{13} & 0 & r_{13} \\
0 & 1 & 0 \\
r_{13} & 0 & t_{13}
\end{array}\right)\left(\begin{array}{ccc}
t_{12} & r_{12} & 0 \\
r_{12} & t_{12} & 0 \\
0 & 0 & 1
\end{array}\right)=\left(\begin{array}{ccc}
t_{12} & r_{12} & 0 \\
r_{12} & t_{12} & 0 \\
0 & 0 & 1
\end{array}\right)\left(\begin{array}{ccc}
t_{13} & 0 & r_{13} \\
0 & 1 & 0 \\
r_{13} & 0 & t_{13}
\end{array}\right)\left(\begin{array}{ccc}
1 & 0 & 0 \\
0 & t_{23} & r_{23} \\
0 & r_{23} & t_{23}
\end{array}\right) \\
\Longrightarrow\left(\begin{array}{ccc}
t_{12} t_{13} & r_{13} \\
t_{23} r_{12}+t_{12} r_{13} r_{23} & t_{12} t_{23}+r_{12} r_{13} r_{23} & t_{13} r_{23} \\
t_{12} t_{23} r_{13}+r_{12} r_{23} & t_{23} r_{12} r_{13}+t_{12} r_{23} & t_{13} t_{23}
\end{array}\right)=\left(\begin{array}{ccc}
t_{12} t_{13} & t_{23} r_{12}+t_{12} r_{13} r_{23} & t_{12} t_{23} r_{13}+r_{12} r_{23} \\
t_{13} r_{12} & t_{12} t_{23}+r_{12} r_{13} r_{23} & t_{23} r_{12} r_{13}+t_{12} r_{23} \\
r_{13} & t_{13} r_{23} & t_{13} t_{23}
\end{array}\right)
\end{gathered}
$$

We see that the conditions simplify to two equations.

$$
\begin{gathered}
t_{13} r_{12}=t_{23} r_{12}+t_{12} r_{13} r_{23} \\
r_{13}=t_{12} t_{23} r_{13}+r_{12} r_{23}
\end{gathered}
$$

Writing $x=k_{1}-k_{2}, y=k_{2}-k_{3} \Longrightarrow x+y=k_{1}-k_{3}$, we have

$$
\begin{gathered}
t(x+y) r(x)=t(y) r(x)+t(x) r(x+y) r(y) \\
r(x+y)=t(x) t(y) r(x+y)+r(x) r(y)
\end{gathered}
$$

We also have $S(k) S(-k)=I$, which implies that

$$
t(x) t(-x)+r(x) r(-x)=1
$$




$$
t(x) r(-x)+r(x) t(-x)=0
$$

Now, we try to find a form for $t(k)$ and $r(k)$. First, we notice from the above two equations that, at $x=0, t(0)=0$ and $r(0)=1$. Since we know the form of the two amplitudes at $x=0$, we can expand the initial two equations about $y$ to obtain the following.

$$
\begin{aligned}
r(x+y)=t(x) t(y) r & (x+y)+r(x) r(y) \\
& \Longrightarrow r(x)+y r^{\prime}(x)=t(x)\left[r(x)+y r^{\prime}(x)\right] t^{\prime}(0) y+r(x)\left[1+r^{\prime}(0) y\right]
\end{aligned}
$$

Comparing the coefficients of $y$, we get

$$
r^{\prime}(x)=r(x)\left[t^{\prime}(0) t(x)+r^{\prime}(0)\right]
$$

Similarly,

$$
\begin{aligned}
t(x+y) r(x)=t & (y) r(x)+t(x) r(x+y) r(y) \\
& \Longrightarrow\left[t(x)+y t^{\prime}(x)\right] r(x)=r(x) t^{\prime}(0) y+t(x)\left[r(x)+y r^{\prime}(x)\right]\left[1+r^{\prime}(0) y\right]
\end{aligned}
$$

Substituting the expression for $r^{\prime}(x)$ from equation (4.31), we get

$$
t^{\prime}(x)=t^{\prime}(0)\left[1+t^{2}(x)\right]+2 r^{\prime}(0) t(x)
$$

We define $r(0)=i \cot \lambda$ and integrate both equations to get

$$
\begin{aligned}
& t(x)=\frac{\sin (i x)}{\sin (\lambda-i x)} \\
& r(x)=\frac{\sin \lambda}{\sin (\lambda-i x)}
\end{aligned}
$$

Hence,

$$
T(k)=-e^{-i \theta(k)} \frac{\sin (i k)}{\sin (\lambda-i k)}
$$




$$
R(k)=-e^{-i \theta(k)} \frac{\sin \lambda}{\sin (\lambda-i k)}
$$

We see that scaling by a factor $\beta$ doesn't alter the differential equations that $t(x)$ and $r(x)$ satisfy. That is, we still have

$$
\begin{gathered}
t^{\prime}(\beta x)=t^{\prime}(0)\left[1+t^{2}(\beta x)\right]+2 r^{\prime}(0) t(\beta x) \\
r^{\prime}(\beta x)=r(\beta x)\left[t^{\prime}(0) t(\beta x)+r^{\prime}(0)\right]
\end{gathered}
$$

If we take $\beta=0$, we get

$$
t^{\prime}(0)=t^{\prime}(0)\left[1+t^{2}(0)\right]+2 r^{\prime}(0) t(0)
$$

which is obviously true since $t(0)=0$. We also have

$$
r^{\prime}(0)=r(0)\left[t^{\prime}(0) t(0)+r^{\prime}(0)\right]
$$

which is again correct since $r(0)=1$ and $t(0)=0$.

Hence, we can scale by a factor to write the expressions for the reflection and transmission amplitudes as

$$
t(\kappa)=\frac{\kappa}{\kappa+i}, \quad r(\kappa)=\frac{-i}{\kappa+i}
$$

Using the S-matrix representation as before, with transmission amplitudes lying on the diagonal, we can see that, if we have a permutation matrix $Q=\left(\begin{array}{ll}0 & 1 \\ 1 & 0\end{array}\right)$, we can write the scattering matrix

$$
S(k)=T(k)+R(k) Q=\left(\begin{array}{cc}
T(k) & R(k) \\
R(k) & T(k)
\end{array}\right)
$$

The scattering matrix then becomes

$$
S(\kappa)=T(\kappa)+R(\kappa) Q=-e^{-i \theta(k)} \frac{\kappa-i}{\kappa+i}
$$


The fact that $t(k)$ is similar in form to $g(\lambda)$ and $r(k)$ is similar to the reciprocal of $f(\lambda)$ is in keeping with the derivation in the previous chapter and it will now be shown that the exchange relations themselves are exactly the same, confirming the validity of the magnon interpretation and the correspondence between the discussions in the previous chapter and this one.

Just as how the monodromy operator corresponds to a translation around the ring, we see that the product of scattering matrices can produce a similar action on the particles. Taking the particle with momentum $k_{j}$ to be scattered and the eigenstate to be $\Psi$, periodicity implies that

$$
\begin{aligned}
e^{i k_{j} L} S_{j, j-1} S_{j, j-2} \ldots S_{j, 1} S_{j, N} S_{j, N-1} \ldots S_{j, 1} \Psi=\Psi & \\
& \Longrightarrow e^{i k_{j} L} S_{j, j-1} S_{j, j-2} \ldots S_{j, 1} S_{j, N} S_{j, N-1} \ldots S_{j, 1}=1
\end{aligned}
$$

We introduce a 'ghost' particle in the system with momentum, say, $k_{0} \equiv \kappa$ and note that the operator defined as a product of scattering operators will take this ghost particle around the ring.

$$
\tau(\kappa)=s_{0, N}\left(\kappa-k_{N}\right) s_{0, N}\left(\kappa-k_{N-1}\right) \ldots s_{0, N}\left(\kappa-k_{1}\right)
$$

We take the particles on the ring to all have up spins, on the whole. The creation operator for the spin chain $B(\lambda)$ is equivalent to an injection of a down spin into the ring. This can be accomplished by assuming the ghost particle to enter the ring with a down spin and exchange spins with one of the up spins in the ring and leave as an up spin.

The particle on a chain problem is therefore very similar to the previously discussed spin chain problem with the R-matrix $R(\lambda-\mu)$ replaced by the reduced scattering matrix $s\left(\kappa-k_{i}\right)$ and the transfer operator $T(\lambda)$ replaced by $\tau(\kappa)$. 
The RTT relation from equation (3.34) is equivalent to the following equation for the particle chain.

$$
\begin{gathered}
s\left(\kappa-\kappa^{\prime}\right) \tau\left(\kappa^{\prime}\right) \tau(\kappa)=\tau(\kappa) \tau(\kappa) s\left(\kappa-\kappa^{\prime}\right) \\
\Longrightarrow \tau\left(\kappa^{\prime}\right) \tau(\kappa)=s\left(\kappa^{\prime}-\kappa\right) \tau(\kappa) \tau(\kappa) s\left(\kappa-\kappa^{\prime}\right)
\end{gathered}
$$

since $s\left(\kappa-\kappa^{\prime}\right) s\left(\kappa^{\prime}-\kappa\right)=1$.

Due to the above equation, we have $\left[\operatorname{tr}\left(\tau\left(\kappa^{\prime}\right)\right), \operatorname{tr}(\tau(\kappa))\right]=0$ using the cyclic property of the trace.

Just like the transfer operator in the spin chain, we can define $\Xi(\kappa)=\operatorname{tr}(\tau(\kappa))$. Since the $\Xi \mathrm{s}$ commute, they share a set of eigenstates which we can find by using the same methodology as in the spin chain.

We note that we can expand $\tau(\kappa)$ as a $2 \times 2$ matrix $\tau(\kappa)=\left(\begin{array}{ll}A(\kappa) & B(\kappa) \\ C(\kappa) & D(\kappa)\end{array}\right)$. Now, expanding the elements of $\tau(\kappa)$ and $s(\kappa)$ just as we did in the spin chain treatment, we get similar relations between the elements of $\tau(\kappa)$, some of which are as follows.

$$
\begin{gathered}
{\left[B(\kappa), B\left(\kappa^{\prime}\right)\right]=0} \\
A(\kappa) B\left(\kappa^{\prime}\right)=\frac{1}{t\left(\kappa^{\prime}-\kappa\right)} B\left(\kappa^{\prime}\right) A(\kappa)-\frac{r\left(\kappa^{\prime}-\kappa\right)}{t\left(\kappa^{\prime}-\kappa\right)} B(\kappa) A\left(\kappa^{\prime}\right) \\
D(\kappa) B\left(\kappa^{\prime}\right)=\frac{1}{t\left(\kappa-\kappa^{\prime}\right)} D\left(\kappa^{\prime}\right) A(\kappa)-\frac{r\left(\kappa-\kappa^{\prime}\right)}{t\left(\kappa-\kappa^{\prime}\right)} D(\kappa) A\left(\kappa^{\prime}\right)
\end{gathered}
$$

Substituting the expressions for $t(\kappa)$ and $r(\kappa$ from equation (4.32) into the last two equations, we get

$$
\begin{aligned}
& A(\kappa) B\left(\kappa^{\prime}\right)=\frac{\kappa-\kappa^{\prime}-i}{\kappa-\kappa^{\prime}} B\left(\kappa^{\prime}\right) A(\kappa)+\frac{i}{\kappa-\kappa^{\prime}} B(\kappa) A\left(\kappa^{\prime}\right) \\
& D(\kappa) B\left(\kappa^{\prime}\right)=\frac{\kappa-\kappa^{\prime}+i}{\kappa-\kappa^{\prime}} D\left(\kappa^{\prime}\right) A(\kappa)-\frac{i}{\kappa-\kappa^{\prime}} D(\kappa) A\left(\kappa^{\prime}\right)
\end{aligned}
$$


These equations are identical to the exchange relations obtained in equations (3.56), (3.57), and (3.58). Following the same treatment as in the previous chapter, the eigenfunctions can be derived.

Throughout the course of the above discussion, the only constraint on the system was that it scattered without diffraction. Aside from this, there is no other requirement or assumption required to derive the above equations. This simplicity truly showcases the power of the Bethe Ansatz, in the sense that it can be applied to any class of systems which satisfy the Yang-Baxter equation, which is linked to diffractionless scattering. Now that we have elaborately obtained a form for the eigenfunctions, momenta, energies, and scattering amplitudes of the system, it would appear that this treatment is now complete. However, a crucial discussion that is yet to be had is about the nature of the interacting potential between the particles we have considered above.

\subsection{The Two-body Problem with a Delta Potential}

An intuitive guess for the interacting potential of the system that has been studied is a delta potential, since the model only allows nearest-neighbour interactions. If we solve the onedimensional two-body problem for a delta potential and obtain the scattering amplitude, we can compare this with the form obtained in the discussion of the Coordinate Bethe Ansatz and see whether the potential is of a delta form.

The time-independent Schrodinger equation for the two-body problem with equal masses is

$$
-\frac{1}{2}\left(\frac{\partial^{2}}{\partial x_{1}^{2}}+\frac{\partial^{2}}{\partial x_{2}^{2}}\right) \Psi+v\left(x_{2}-x_{1}\right) \Psi=E \Psi
$$

Here, $E=\frac{k_{1}^{2}}{2}+\frac{k_{2}^{2}}{2}$ where $k_{1}$ and $k_{2}$ are the momenta of the particles.

Using the coordinates $r=x_{2}-x_{1}, k=k_{1}-k_{2}$ and $R=x_{1}+x_{2}, K=k_{1}+k_{2}$, we can separate the centre of mass components of the wavefunction by writing $\Psi\left(x_{1}, x_{2}\right)=e^{i K R / 2} \psi(r)$. The Schrodinger equation can then be rewritten as

$$
-\psi^{\prime \prime}(r)+v(r) \psi(r)=\left(\frac{k}{2}\right)^{2} \psi(r)
$$

Only considering symmetric potentials, the wavefunctions can either be even or odd, say, $\psi_{ \pm}(r)$. The phase shift between the incoming and outgoing wavefunctions is given by $\theta_{ \pm}(k)$. The forms of the even and odd wavefunctions are, therefore, 


$$
\psi_{ \pm}(r)= \begin{cases}e^{-i k r / 2}-e^{i k r / 2-i \theta_{ \pm}(k)} & r \rightarrow \infty \\ \pm\left[e^{i k r / 2}-e^{-i k r / 2-i \theta_{ \pm}(k)}\right] & r \rightarrow-\infty\end{cases}
$$

The total wavefunction must be a superposition of the odd and even wavefunctions. Say,

$$
\frac{1}{2}\left(\psi_{+}(r)+\psi_{-}(r)\right)= \begin{cases}e^{-i k r / 2}-e^{i k r / 2}\left\{e^{-i \theta_{+}(k)}+e^{-i \theta_{-}(k)}\right\} / 2 & r \rightarrow \infty \\ e^{-i k r / 2}\left\{e^{-i \theta_{-}(k)}-e^{-i \theta_{-}(k)}\right\} / 2 & r \rightarrow-\infty\end{cases}
$$

We infer from the above equation that the reflection and transmission amplitudes are as follows.

$$
\begin{gathered}
R(k)=-\frac{e^{-i \theta_{+}(k)}+e^{-i \theta_{-}(k)}}{2} \\
T(k)=\frac{e^{-i \theta_{-}(k)}-e^{-i \theta_{+}(k)}}{2}
\end{gathered}
$$

Using the S-matrix representation as before, with transmission amplitudes lying on the diagonal, we can see that, if we have a permutation matrix $Q=\left(\begin{array}{ll}0 & 1 \\ 1 & 0\end{array}\right)$, we can write the scattering matrix

$$
S(k)=T(k)+R(k) Q=\left(\begin{array}{cc}
T(k) & R(k) \\
R(k) & T(k)
\end{array}\right)
$$

Since we have an expression for the reflection and transmission amplitudes in terms of the phase shift, we can calculate the phase shift and subsequently the scattering matrix for the delta potential.

Assume the potential is of the form $v(r)=c \delta(r)$, where $c$ is some constant.

Since the delta function is only non-zero at the origin and the odd wavefunction is zero at the origin, the potential has no effect and the equation of motion becomes

$$
\psi_{-}^{\prime \prime}(r)=-\frac{k^{2}}{4} \psi_{-}(r) \Longrightarrow \psi_{-}(r)=e^{-i k r / 2}-e^{i k r / 2}=-2 i \sin (k r / 2)
$$

Since the potential has no effect on the wavefunction, the antisymmetric phase shift $\theta_{-}(k)=$ 0 .

The equation of motion for the even function is now as follows. 


$$
\begin{gathered}
-\psi_{+}^{\prime \prime}(r)+c \delta(r) \psi_{+}(r)=\left(\frac{k}{2}\right)^{2} \psi_{+}(r) \Longrightarrow \int_{-\infty}^{\infty}-\psi_{+}^{\prime \prime}(r) d r+\int_{-\infty}^{\infty} c \delta(r) \psi_{+}(r) d r=\int_{-\infty}^{\infty} \frac{k^{2}}{4} \psi_{+}(r) d r \\
\Longrightarrow 2 \int_{0}^{\infty} \psi_{+}^{\prime \prime}(r) d r=c \psi_{+}(0) \\
\Longrightarrow 2 \psi_{+}^{\prime}\left(0^{+}\right)=c \psi_{+}(0)
\end{gathered}
$$

We write the wavefunction to be as follows, since the potential is asymptotic everywhere apart from the origin.

$$
\psi_{+}(r)=-2 i \sin \left(k|r| / 2-\theta_{+} / 2\right)
$$

$$
\psi_{+}^{\prime}(r)=-i k \cos \left(k r / 2-\theta_{+} / 2\right), \quad r>0 \Longrightarrow \frac{\psi_{+}^{\prime}(r)}{\psi_{+}(r)}=\frac{k}{2} \cot \left(k r / 2-\theta_{+} / 2\right), \quad r>0
$$

From equation (4.53), we have

$$
\frac{\psi_{+}^{\prime}(0)}{\psi_{+}(0)}=\frac{c}{2}=\frac{k}{2} \cot \left(-\theta_{+} / 2\right)=-\frac{k}{2} \cot \left(\theta_{+} / 2\right) \Longrightarrow \theta_{+}(k)=-2 \tan ^{-1}\left(\frac{k}{c}\right)
$$

This is equivalent to saying that

$$
\theta_{+}(k)=-\frac{1}{i} \log \frac{k / 2 c+i / 2}{k / 2 c-i / 2}=-\frac{1}{i} \log \frac{c+i k}{c-i k} \Longrightarrow e^{-i \theta_{+}}=\frac{c+i k}{c-i k}
$$

We also have $e^{-i \theta_{-}}=1$. Using equations (4.48) and (4.49), we see that

$$
\begin{gathered}
R(k)=-\frac{1}{2}\left(1+\frac{c+i k}{c-i k}\right)=-\frac{c}{c-i k}=-\frac{i c}{k+i c} \\
T(k)=\frac{1}{2}\left(1-\frac{c+i k}{c-i k}\right)=\frac{-i k}{c-i k}=\frac{k}{k+i c}
\end{gathered}
$$

Using equation (4.50), we have

$$
S(k)=T(k)+R(k) Q=\frac{k-i c Q}{k+i c}=\frac{k_{1}-k_{2}-i c Q}{k_{1}-k_{2}+i c}
$$


The constant $c$ is just a length scale and can be set to 1 . Hence,

$$
S(k)=\frac{k_{2}-k_{1}-i Q}{k_{2}-k_{1}+i}
$$

This form of the S-matrix is identical to the scattering amplitude obtained through the Coordinate Bethe Ansatz in equation (3.15). It is also the same as the scattering matrix obtained for the scattering of complexes or bound states of type 0 (3.106) up to a scale and to the form obtained in the previous discussion of the system satisfying the Yang-Baxter Equation (4.34). Thus, we see that the form of the scattering matrix retains its form regardless of the physical interpretation of magnons or bound states. 


\section{Chapter 5}

\section{Summary and Conclusion}

\subsection{Summary}

Integrable systems are a very special class of models that have garnered great interest in the last 75 years. In the course of this thesis, the general procedure of finding out whether a system is integrable or not and, if it is, the method of solving it have been closely studied using a series of established works with the backdrop of the one-dimensional XXX-1/2 spin chain model, which is a perfect starting point to understand integrability and the Algebraic Bethe Ansatz.

Owing to being a simple model to visualise, the expressions for the energy and the scattering amplitude of the model were easily derived using the Coordinate Bethe Ansatz, which provided an introduction to the idea of 'guessing' the form of a wavefunction, since imposing periodicity made a plane wave seem like a likely solution. We use the Lax formulation to establish that an integrable system has a family of as many commuting operators in involution as there are degrees of freedom with one of them being the Hamiltonian. We saw that the existence of a Lax pair enabled one to find $N$ constants of motion. In order for these to be in involution, we defined an R-matrix which helped the Lax operators commute. We realised that there was no a priori way to derive the Lax operator or R-matrix for a system, but could be filtered through a trial-and-error process. Once valid forms for both the Lax operator and R-matrix are proposed, the Fundamental Commutation Relations (FCRs) that they obey can be derived, as was done for the isotropic spin chain. The monodromy operator was defined to transport a spin once around the ring completely. The FCRs allowed us to show that the Hamiltonian belonged to the family of the trace of monodromy, and that there are $N$ such operators. Moreover, they allowed us to obtain exchange relations between the elements of the monodromy matrix. Defining the vacuum state over the quantum space as all up spins, it was realised that two of these elements corresponded to the creation and destruction operators in the harmonic oscillator model. By acting on the vacuum state with the creation operator at a particular site, an up spin was 
essentially flipped. By flipping $l(l \leq N / 2)$ such spins, we had an expression for the general state. Constraining the arbitrary parameters or 'rapidities', we could make the vector an eigenstate of the transfer operator and hence, of the Hamiltonian. The constraining set of $l$ equations are called the Bethe Ansatz Equations. We found the energy and momentum eigenvalues to be identical to those obtained by the Coordinate Bethe Ansatz. The fact that the flipping of even one spin could allow an energy and momentum to be calculated implied that the flipping of the spin created a quasiparticle called a 'magnon' with a momentum and energy of its own.

Proceeding to study low-lying excited states in the thermodynamic limit, we saw that the rapidities could be segregated into groups with the same real part but separated equally by $\sqrt{-1}$ when arranged in order. The collective energy of one of these groups, or 'complexes', was seen to be lesser than the sum of the energies corresponding to the individual rapidities in the group while the dispersion relation was maintained. Hence, we inferred that these complexes behaved like bound states and their momentum and energy were derived as were their scattering matrices with other complexes. Writing the Bethe Ansatz Equations in a logarithmic form, we realised that the rapidities could be uniquely mapped to quantum numbers. We counted the total number of Bethe vectors using this interpretation and found this to be exactly $2^{N}$, which was the dimension of the model's Hilbert space, confirming the bound state hypothesis.

Consequently, it became imperative to study the first few bound states. In the antiferromagnetic case, we saw that there could only be an even number of particles in a state. Setting the ground state to have an energy of zero by definition, we showed that the energies of the triplet and singlet states were the same and equal to the sum of the energies of two magnons. The contribution of the complex perfectly canceled out in each case. Hence, the energies of the excited states were established to purely be sums of the energies corresponding to the flipped spins, or the magnon energies.

Probing the magnon interpretation further, the two-body scattering problem was studied and it was seen that integrability is directly linked to diffractionless scattering. If the scattering matrices satisfy the Yang-Baxter Equation, the system is integrable and an exact solution could be found. Using the Yang-Baxter Equation, expressions for the transmission and reflection amplitudes were obtained. It was further observed that by putting these particles on a chain and treating the scattering matrix like the R-matrix, the exchange relations obtained were identical to those obtained using the Algebraic Bethe Ansatz, implying that the only constraint equation needed to obtain exact eigenfunctions was the Yang-Baxter Equation.

Finally, a two-body scattering problem with a delta potential was shown to have the same scattering amplitude as that obtained from the coordinate ansatz as well as the bound state and magnon interpretations, implying that the spin chain Hamiltonian corresponds to a delta interaction. The analysis of the model was therefore complete. 


\subsection{Conclusion}

All of the above results imply that there is a great deal of consistency in the underlying equations describing an integrable system. The methodology of solving for the wavefunctions of any system using the Algebraic Bethe Ansatz is now very clear. Recognising the fact that scattering without diffraction is a necessary condition for integrability, it first needs to be verified that the scattering matrix of the system satisfies the Yang-Baxter Equation. Having established this, forms for the Lax operator and R-matrix are found and an ansatz for the general state is made. Constraining these states to be eigenfunctions of any and therefore all of the $N$ commuting operators of the model, the Bethe Ansatz Equations are obtained and the forms of the eigenstates as well as their energy and momenta are found.

What makes the study of integrable systems so alluring is the fact that they all obey the same single equation, which warrants the use of the Bethe Ansatz. The Yang-Baxter Equation itself simultaneously verifies that systems have exact solutions and relates the algebraic equations to scattering problems. As we have seen, the XXX-1/2 model is one of the simplest solutions to diffractionless scattering. However, even introducing complications to these models does not change the fact that the Bethe Ansatz can always be used as a tool to solve for their wavefunctions. We linked the spin chain to particles and bound states and after a great deal of mathematical manipulation, arrived at the exact same results in every case. This innate symmetry to integrable systems now has a way to be physically and mathematically given form, and that is one of the most important inferences from this discussion. However, it is important to note that there is no standard method to obtain the forms of the Lax operator and R-matrix and these can only be found through trial-and-error. At the same time, it is important to note that once we obtain forms for these operators, the Algebraic Bethe Ansatz is the ideal technique to find the solutions of this class of models. 


\section{Appendix A}

\section{Proofs of Select Equations from the Analysis of $\mathrm{XXX}_{1 / 2}$ Model}

\section{A.1 Proof of RLL Relation (3.28)}

We need to prove that $R_{a_{1}, a_{2}}(\lambda-\mu) L_{n, a_{1}}(\lambda) L_{n, a_{2}}(\mu)=L_{n, a_{2}}(\mu) L_{n, a_{1}}(\lambda) R_{a_{1}, a_{2}}(\lambda-\mu)$.

It must be noted that since $\mathcal{H}, V_{1}$ and $V_{2}$ are defined on $\mathrm{C}^{2}$, they all have the same identity operator, and we will henceforth denote the cross-product of identity operators $I \otimes I \otimes I$ by I.

We start from the left hand side.

$$
\begin{gathered}
R_{a_{1}, a_{2}}(\lambda-\mu) L_{n, a_{1}}(\lambda) L_{n, a_{2}}(\mu) \\
=\left[(\lambda-\mu) I+i P_{a_{1}, a_{2}}\right]\left[\left(\lambda-\frac{i}{2}\right) I+i P_{n, a_{1}}\right]\left[\left(\mu-\frac{i}{2}\right) I+i P_{n, a_{2}}\right] \\
=\left[(\lambda-\mu) I+i P_{a_{1}, a_{2}}\right]\left[\left(\lambda-\frac{i}{2}\right)\left(\mu-\frac{i}{2}\right) I+i\left(\lambda-\frac{i}{2}\right) P_{n, a_{2}}+i\left(\mu-\frac{i}{2}\right) P_{n, a_{1}}-P_{n, a_{1}} P_{n, a_{2}}\right] \\
=\left[(\lambda-\mu)\left(\lambda-\frac{i}{2}\right)\left(\mu-\frac{i}{2}\right) I+i P_{a_{1}, a_{2}}\right]\left[\left(\lambda-\frac{i}{2}\right)\left(\mu-\frac{i}{2}\right) I+i(\lambda-\mu)\left(\lambda-\frac{i}{2}\right) P_{n, a_{2}}\right. \\
\left.+i(\lambda-\mu)\left(\mu-\frac{i}{2}\right) P_{n, a_{1}}+i\left(\lambda-\frac{i}{2}\right)\left(\mu-\frac{i}{2}\right) P_{a_{1}, a_{2}}\right] \\
-\left[\left(\lambda-\frac{i}{2}\right) P_{a_{1}, a_{2}} P_{n, a_{2}}+\left(\mu-\frac{i}{2}\right) P_{a_{1}, a_{2}} P_{n, a_{1}}+(\lambda-\mu) P_{n, a_{1}} P_{n, a_{2}}+i P_{a_{1}, a_{2}} P_{n, a_{1}} P_{n, a_{2}}\right]
\end{gathered}
$$




$$
\begin{aligned}
& =\left[(\lambda-\mu)\left(\lambda-\frac{i}{2}\right)\left(\mu-\frac{i}{2}\right) I+i P_{a_{1}, a_{2}}\right]\left[\left(\lambda-\frac{i}{2}\right)\left(\mu-\frac{i}{2}\right) I+i(\lambda-\mu)\left(\lambda-\frac{i}{2}\right) P_{n, a_{2}}\right. \\
& \left.+i(\lambda-\mu)\left(\mu-\frac{i}{2}\right) P_{n, a_{1}}+i\left(\lambda-\frac{i}{2}\right)\left(\mu-\frac{i}{2}\right) P_{a_{1}, a_{2}}\right] \\
& -\left[\lambda P_{a_{1}, a_{2}} P_{n, a_{2}}+\lambda P_{n, a_{1}} P_{n, a_{2}}+\mu P_{a_{1}, a_{2}} P_{n, a_{1}}-\mu P_{n, a_{1}} P_{n, a_{2}}+i P_{a_{1}, a_{2}} P_{n, a_{1}} P_{n, a_{2}}-\frac{i}{2} P_{a_{1}, a_{2}} P_{n, a_{2}}-\frac{i}{2} P_{a_{1}, a_{2}} P_{n, a_{1}}\right] \\
& =\left[(\lambda-\mu)\left(\lambda-\frac{i}{2}\right)\left(\mu-\frac{i}{2}\right) I+i P_{a_{1}, a_{2}}\right]\left[\left(\lambda-\frac{i}{2}\right)\left(\mu-\frac{i}{2}\right) I+i(\lambda-\mu)\left(\lambda-\frac{i}{2}\right) P_{n, a_{2}}\right. \\
& \left.+i(\lambda-\mu)\left(\mu-\frac{i}{2}\right) P_{n, a_{1}}+i\left(\lambda-\frac{i}{2}\right)\left(\mu-\frac{i}{2}\right) P_{a_{1}, a_{2}}\right] \\
& -\left[\lambda\left(P_{a_{1}, a_{2}} P_{n, a_{2}}+P_{n, a_{1}} P_{n, a_{2}}\right)+\mu P_{n, a_{1}} P_{n, a_{2}}-\mu P_{n, a_{1}} P_{n, a_{2}}+i P_{a_{1}, a_{2}} P_{n, a_{2}} P_{a_{2}, a_{1}}-\frac{i}{2} P_{a_{1}, a_{2}} P_{n, a_{2}}-\frac{i}{2} P_{a_{1}, a_{2}} P_{n, a_{1}}\right] \\
& =\left[(\lambda-\mu)\left(\lambda-\frac{i}{2}\right)\left(\mu-\frac{i}{2}\right) I+i P_{a_{1}, a_{2}}\right]\left[\left(\lambda-\frac{i}{2}\right)\left(\mu-\frac{i}{2}\right) I+i(\lambda-\mu)\left(\lambda-\frac{i}{2}\right) P_{n, a_{2}}\right. \\
& \left.+i(\lambda-\mu)\left(\mu-\frac{i}{2}\right) P_{n, a_{1}}+i\left(\lambda-\frac{i}{2}\right)\left(\mu-\frac{i}{2}\right) P_{a_{1}, a_{2}}\right] \\
& -\left[\lambda\left(P_{n, a_{2}} P_{n, a_{1}}+P_{n, a_{1}} P_{n, a_{2}}\right)+i P_{a_{1}, a_{2}} P_{n, a_{2}} P_{a_{2}, a_{1}}-\frac{i}{2} P_{a_{1}, a_{2}} P_{n, a_{2}}-\frac{i}{2} P_{a_{1}, a_{2}} P_{n, a_{1}}\right]
\end{aligned}
$$

Similarly proceeding on the right hand side,

$$
\begin{gathered}
L_{n, a_{2}}(\mu) L_{n, a_{1}}(\lambda) R_{a_{1}, a_{2}}(\lambda-\mu) \\
=\left[\left(\mu-\frac{i}{2}\right) I+i P_{n, a_{2}}\right]\left[\left(\lambda-\frac{i}{2}\right) I+i P_{n, a_{1}}\right]\left[(\lambda-\mu) I+i P_{a_{1}, a_{2}}\right] \\
=\left[\left(\lambda-\frac{i}{2}\right)\left(\mu-\frac{i}{2}\right) I+i\left(\lambda-\frac{i}{2}\right) P_{n, a_{2}}+i\left(\mu-\frac{i}{2}\right) P_{n, a_{1}}-P_{n, a_{2}} P_{n, a_{1}}\right]\left[(\lambda-\mu) I+i P_{a_{1}, a_{2}}\right] \\
=\left[(\lambda-\mu)\left(\lambda-\frac{i}{2}\right)\left(\mu-\frac{i}{2}\right) I+i P_{a_{1}, a_{2}}\right]\left[\left(\lambda-\frac{i}{2}\right)\left(\mu-\frac{i}{2}\right) I+i(\lambda-\mu)\left(\lambda-\frac{i}{2}\right) P_{n, a_{2}}\right. \\
\left.+i(\lambda-\mu)\left(\mu-\frac{i}{2}\right) P_{n, a_{1}}+i\left(\lambda-\frac{i}{2}\right)\left(\mu-\frac{i}{2}\right) P_{a_{1}, a_{2}}\right] \\
-\left[\left(\lambda-\frac{i}{2}\right) P_{n, a_{2}} P_{a_{1}, a_{2}}+\left(\mu-\frac{i}{2}\right) P_{n, a_{1}} P_{a_{1}, a_{2}}+(\lambda-\mu) P_{n, a_{2}} P_{n, a_{1}}+i P_{n, a_{2}} P_{a_{1}, a_{2}} P_{n, a_{1}}\right]
\end{gathered}
$$




$$
\begin{gathered}
=\left[(\lambda-\mu)\left(\lambda-\frac{i}{2}\right)\left(\mu-\frac{i}{2}\right) I+i P_{a_{1}, a_{2}}\right]\left[\left(\lambda-\frac{i}{2}\right)\left(\mu-\frac{i}{2}\right) I+i(\lambda-\mu)\left(\lambda-\frac{i}{2}\right) P_{n, a_{2}}\right. \\
\left.+i(\lambda-\mu)\left(\mu-\frac{i}{2}\right) P_{n, a_{1}}+i\left(\lambda-\frac{i}{2}\right)\left(\mu-\frac{i}{2}\right) P_{a_{1}, a_{2}}\right] \\
\quad-\left[\left(\lambda-\frac{i}{2}\right) P_{n, a_{2}} P_{a_{1}, a_{2}}+\left(\mu-\frac{i}{2}\right) P_{n, a_{1}} P_{a_{1}, a_{2}}+(\lambda-\mu) P_{n, a_{2}} P_{n, a_{1}}+i P_{n, a_{2}} P_{a_{1}, a_{2}} P_{n, a_{1}}\right]
\end{gathered}
$$

$$
\begin{aligned}
& =\left[(\lambda-\mu)\left(\lambda-\frac{i}{2}\right)\left(\mu-\frac{i}{2}\right) I+i P_{a_{1}, a_{2}}\right]\left[\left(\lambda-\frac{i}{2}\right)\left(\mu-\frac{i}{2}\right) I+i(\lambda-\mu)\left(\lambda-\frac{i}{2}\right) P_{n, a_{2}}\right. \\
& \left.+i(\lambda-\mu)\left(\mu-\frac{i}{2}\right) P_{n, a_{1}}+i\left(\lambda-\frac{i}{2}\right)\left(\mu-\frac{i}{2}\right) P_{a_{1}, a_{2}}\right] \\
& -\left[\lambda P_{n, a_{2}} P_{a_{1}, a_{2}}+\lambda P_{n, a_{2}} P_{n, a_{1}}+\mu P_{n, a_{1}} P_{a_{1}, a_{2}}-\mu P_{n, a_{2}} P_{n, a_{1}}+i P_{n, a_{2}} P_{n, a_{1}} P_{a_{1}, a_{2}}-\frac{i}{2} P_{n, a_{2}} P_{a_{1}, a_{2}}-\frac{i}{2} P_{n, a_{1}} P_{a_{1}, a_{2}}\right] \\
& =\left[(\lambda-\mu)\left(\lambda-\frac{i}{2}\right)\left(\mu-\frac{i}{2}\right) I+i P_{a_{1}, a_{2}}\right]\left[\left(\lambda-\frac{i}{2}\right)\left(\mu-\frac{i}{2}\right) I+i(\lambda-\mu)\left(\lambda-\frac{i}{2}\right) P_{n, a_{2}}\right. \\
& \left.+i(\lambda-\mu)\left(\mu-\frac{i}{2}\right) P_{n, a_{1}}+i\left(\lambda-\frac{i}{2}\right)\left(\mu-\frac{i}{2}\right) P_{a_{1}, a_{2}}\right] \\
& -\left[\lambda P_{n, a_{2}} P_{a_{1}, a_{2}}+\lambda P_{n, a_{2}} P_{n, a_{1}}+\mu P_{n, a_{1}} P_{a_{1}, a_{2}}-\mu P_{n, a_{2}} P_{n, a_{1}}+i P_{n, a_{2}} P_{n, a_{1}} P_{a_{1}, a_{2}}-\frac{i}{2} P_{a_{1}, a_{2}} P_{n, a_{1}}-\frac{i}{2} P_{a_{1}, a_{2}} P_{n, a_{2}}\right] \\
& =\left[(\lambda-\mu)\left(\lambda-\frac{i}{2}\right)\left(\mu-\frac{i}{2}\right) I+i P_{a_{1}, a_{2}}\right]\left[\left(\lambda-\frac{i}{2}\right)\left(\mu-\frac{i}{2}\right) I+i(\lambda-\mu)\left(\lambda-\frac{i}{2}\right) P_{n, a_{2}}\right. \\
& \left.+i(\lambda-\mu)\left(\mu-\frac{i}{2}\right) P_{n, a_{1}}+i\left(\lambda-\frac{i}{2}\right)\left(\mu-\frac{i}{2}\right) P_{a_{1}, a_{2}}\right] \\
& -\left[\lambda\left(P_{n, a_{1}} P_{n, a_{2}}+P_{n, a_{2}} P_{n, a_{1}}\right)+\mu P_{n, a_{2}} P_{n, a_{1}}-\mu P_{n, a_{2}} P_{n, a_{1}}+i P_{a_{2}, a_{1}} P_{n, a_{2}} P_{a_{1}, a_{2}}-\frac{i}{2} P_{a_{1}, a_{2}} P_{n, a_{1}}-\frac{i}{2} P_{a_{1}, a_{2}} P_{n, a_{2}}\right] \\
& =\left[(\lambda-\mu)\left(\lambda-\frac{i}{2}\right)\left(\mu-\frac{i}{2}\right) I+i P_{a_{1}, a_{2}}\right]\left[\left(\lambda-\frac{i}{2}\right)\left(\mu-\frac{i}{2}\right) I+i(\lambda-\mu)\left(\lambda-\frac{i}{2}\right) P_{n, a_{2}}\right. \\
& \left.+i(\lambda-\mu)\left(\mu-\frac{i}{2}\right) P_{n, a_{1}}+i\left(\lambda-\frac{i}{2}\right)\left(\mu-\frac{i}{2}\right) P_{a_{1}, a_{2}}\right] \\
& -\left[\lambda\left(P_{n, a_{1}} P_{n, a_{2}}+P_{n, a_{2}} P_{n, a_{1}}\right)+i P_{a_{1}, a_{2}} P_{n, a_{2}} P_{a_{2}, a_{1}}-\frac{i}{2} P_{a_{1}, a_{2}} P_{n, a_{1}}-\frac{i}{2} P_{a_{1}, a_{2}} P_{n, a_{2}}\right] \\
& =\left[(\lambda-\mu)\left(\lambda-\frac{i}{2}\right)\left(\mu-\frac{i}{2}\right) I+i P_{a_{1}, a_{2}}\right]\left[\left(\lambda-\frac{i}{2}\right)\left(\mu-\frac{i}{2}\right) I+i(\lambda-\mu)\left(\lambda-\frac{i}{2}\right) P_{n, a_{2}}\right. \\
& \left.+i(\lambda-\mu)\left(\mu-\frac{i}{2}\right) P_{n, a_{1}}+i\left(\lambda-\frac{i}{2}\right)\left(\mu-\frac{i}{2}\right) P_{a_{1}, a_{2}}\right] \\
& -\left[\lambda\left(P_{n, a_{2}} P_{n, a_{1}}+P_{n, a_{1}} P_{n, a_{2}}\right)+i P_{a_{1}, a_{2}} P_{n, a_{2}} P_{a_{2}, a_{1}}-\frac{i}{2} P_{a_{1}, a_{2}} P_{n, a_{2}}-\frac{i}{2} P_{a_{1}, a_{2}} P_{n, a_{1}}\right]
\end{aligned}
$$

Clearly, both sides are equal and the relation is proven. 


\section{A.2 Derivation of Exchange Relations (3.56)}

In order to derive the FCR, the relevant operators are represented in $V \otimes V$ and have the following form.

$$
\begin{aligned}
& P=\frac{1}{2}\left(I \otimes I+\sum_{\alpha} \sigma^{\alpha} \otimes \sigma^{\alpha}\right) \\
& =\frac{1}{2}\left(\begin{array}{llll}
1 & 0 & 0 & 0 \\
0 & 1 & 0 & 0 \\
0 & 0 & 1 & 0 \\
0 & 0 & 0 & 1
\end{array}\right)+\frac{1}{2}\left(\begin{array}{llll}
0 & 0 & 0 & 1 \\
0 & 0 & 1 & 0 \\
0 & 1 & 0 & 0 \\
1 & 0 & 0 & 0
\end{array}\right)+\frac{1}{2}\left(\begin{array}{cccc}
0 & 0 & 0 & -1 \\
0 & 0 & 1 & 0 \\
0 & 1 & 0 & 0 \\
-1 & 0 & 0 & 0
\end{array}\right)+\frac{1}{2}\left(\begin{array}{cccc}
1 & 0 & 0 & 0 \\
0 & -1 & 0 & 0 \\
0 & 0 & -1 & 0 \\
0 & 0 & 0 & 1
\end{array}\right) \\
& =\frac{1}{2}\left(\begin{array}{llll}
2 & 0 & 0 & 0 \\
0 & 0 & 2 & 0 \\
0 & 2 & 0 & 0 \\
0 & 0 & 0 & 2
\end{array}\right)=\left(\begin{array}{llll}
1 & 0 & 0 & 0 \\
0 & 0 & 1 & 0 \\
0 & 1 & 0 & 0 \\
0 & 0 & 0 & 1
\end{array}\right) \\
& R(\lambda)=\lambda I+i P \\
& =\left(\begin{array}{cccc}
\lambda+i & 0 & 0 & 0 \\
0 & \lambda & i & 0 \\
0 & i & \lambda & 0 \\
0 & 0 & 0 & \lambda+i
\end{array}\right)=\left(\begin{array}{cccc}
a(\lambda) & 0 & 0 & 0 \\
0 & b(\lambda) & c(\lambda) & 0 \\
0 & c(\lambda) & b(\lambda) & 0 \\
0 & 0 & 0 & a(\lambda)
\end{array}\right) \\
& T_{a_{1}}(\lambda)=T(\lambda) \otimes I_{a_{2}}=\left(\begin{array}{ll}
A(\lambda) \otimes I_{a_{2}} & B(\lambda) \otimes I_{a_{2}} \\
C(\lambda) \otimes I_{a_{2}} & D(\lambda) \otimes I_{a_{2}}
\end{array}\right)=\left(\begin{array}{cccc}
A(\lambda) & 0 & B(\lambda) & 0 \\
0 & A(\lambda) & 0 & B(\lambda) \\
C(\lambda) & 0 & D(\lambda) & 0 \\
0 & C(\lambda) & 0 & D(\lambda)
\end{array}\right) \\
& T_{a_{2}}(\mu)=I_{a_{1}} \otimes T(\mu)=\left(\begin{array}{cc}
T(\mu) & 0 \\
0 & T(\mu)
\end{array}\right)=\left(\begin{array}{cccc}
A(\mu) & B(\mu) & 0 & 0 \\
C(\mu) & D(\mu) & 0 & 0 \\
0 & 0 & A(\mu) & B(\mu) \\
0 & 0 & C(\mu) & D(\mu)
\end{array}\right)
\end{aligned}
$$

Using the RTT relation (3.34) with the above tensor forms, we have

$$
R_{a_{1}, a_{2}}(\lambda-\mu) T_{a_{1}}(\lambda) T_{a_{2}}(\mu)=T_{a_{2}}(\mu) T_{a_{1}}(\lambda) R_{a_{1}, a_{2}}(\lambda-\mu) \Longrightarrow
$$




$$
\begin{aligned}
& \left(\begin{array}{cccc}
a(\lambda) & 0 & 0 & 0 \\
0 & b(\lambda) & c(\lambda) & 0 \\
0 & c(\lambda) & b(\lambda) & 0 \\
0 & 0 & 0 & a(\lambda)
\end{array}\right)\left(\begin{array}{cccc}
A(\lambda) A(\mu) & A(\lambda) B(\mu) & B(\lambda) A(\mu) & B(\lambda) B(\mu) \\
A(\lambda) C(\mu) & A(\lambda) D(\mu) & B(\lambda) C(\mu) & B(\lambda) D(\mu) \\
C(\lambda) A(\mu) & C(\lambda) B(\mu) & D(\lambda) A(\mu) & D(\lambda) B(\mu) \\
C(\lambda) C(\mu) & C(\lambda) D(\mu) & D(\lambda) C(\mu) & D(\lambda) D(\mu)
\end{array}\right) \\
& \left(\begin{array}{llll}
A(\mu) A(\lambda) & B(\mu) A(\lambda) & A(\mu) B(\lambda) & B(\mu) B(\lambda) \\
C(\mu) A(\lambda) & D(\mu) A(\lambda) & C(\mu) B(\lambda) & D(\mu) B(\lambda) \\
A(\mu) C(\lambda) & B(\mu) C(\lambda) & A(\mu) D(\lambda) & B(\mu) D(\lambda) \\
C(\mu) C(\lambda) & D(\mu) C(\lambda) & C(\mu) D(\lambda) & D(\mu) D(\lambda)
\end{array}\right)\left(\begin{array}{cccc}
a(\lambda) & 0 & 0 & 0 \\
0 & b(\lambda) & c(\lambda) & 0 \\
0 & c(\lambda) & b(\lambda) & 0 \\
0 & 0 & 0 & a(\lambda)
\end{array}\right) \Longrightarrow
\end{aligned}
$$

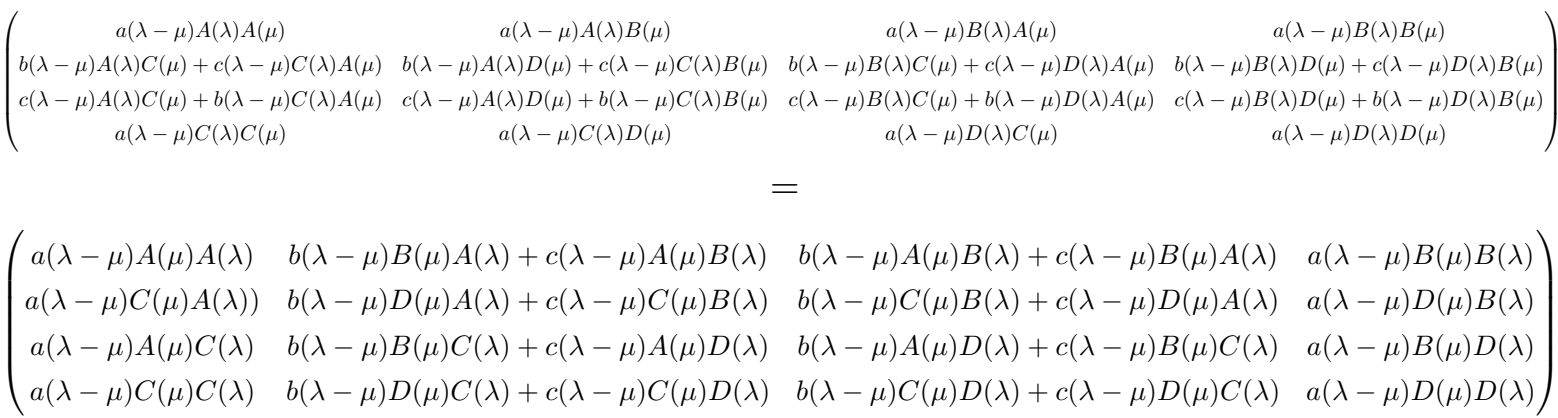

From the above equality, the following are some of the relations obtained.

$$
\begin{gathered}
{[A(\mu), A(\lambda)]=0} \\
{[B(\mu), B(\lambda)]=0} \\
{[C(\mu), C(\lambda)]=0} \\
{[D(\mu), D(\lambda)]=0} \\
a(\lambda) B(\lambda) A(\mu)=b(\lambda-\mu) B(\mu) A(\lambda)+c(\lambda-\mu) A(\mu) B(\lambda) \Longrightarrow \\
A(\lambda) B(\mu)=f(\lambda-\mu) B(\mu) A(\lambda)+g(\lambda-\mu) B(\lambda) A(\mu) \\
c(\lambda-\mu) B(\lambda) D(\mu)+b(\lambda-\mu) D(\lambda) B(\mu)=a(\lambda-\mu) B(\mu) D(\lambda) \Longrightarrow \\
D(\lambda) B(\mu)=h(\lambda-\mu) B(\mu) D(\lambda)+k(\lambda-\mu) B(\lambda) D(\mu)
\end{gathered}
$$$$
\text { where } f(\lambda)=\frac{\lambda-i}{\lambda} \quad ; g(\lambda)=\frac{i}{\lambda} \quad ; h(\lambda)=\frac{\lambda+i}{\lambda} \quad ; k(\lambda)=\frac{-i}{\lambda}
$$ 


\section{A.3 Derivation of form of $M_{j}(\lambda, \lambda)$}

Using induction, it can be shown that the form of $M_{j}(\lambda,\{\lambda\})$ as introduced in equation (3.66) is given by

$$
M_{j}(\lambda,\{\lambda\})=g\left(\lambda-\lambda_{j}\right) \prod_{k \neq j}^{l} f\left(\lambda-\lambda_{j}\right) \alpha^{N}\left(\lambda_{j}\right)
$$

The proof is as follows.

For $j=1$, we have $M_{1}(\lambda,\{\lambda\})=g\left(\lambda-\lambda_{1}\right)$, which is correct since

$$
\begin{gathered}
A(\lambda) \Phi(\{\lambda\})=A(\lambda) B\left(\lambda_{1}\right) \Omega=f\left(\lambda-\lambda_{1}\right) B\left(\lambda_{1}\right) A(\lambda) \Omega+g\left(\lambda-\lambda_{1}\right) B(\lambda) A\left(\lambda_{1}\right) \Omega= \\
\\
f\left(\lambda-\lambda_{1}\right) \alpha^{N}(\lambda) B\left(\lambda_{1}\right) \Omega+g\left(\lambda-\lambda_{1}\right) \alpha^{N}\left(\lambda_{1}\right) B(\lambda) \Omega
\end{gathered}
$$

Now, assuming it is true for $j=l$, that is,

$$
\begin{gathered}
A(\lambda) B\left(\lambda_{1}\right) B\left(\lambda_{2}\right) \ldots B\left(\lambda_{l}\right) \Omega=\prod_{k=1}^{l} f\left(\lambda-\lambda_{k}\right) \alpha^{N}(\lambda) B\left(\lambda_{1}\right) B\left(\lambda_{2}\right) \ldots B\left(\lambda_{l}\right) \Omega+ \\
\left.\sum_{k=1}^{l} M_{k}(\lambda,\{\lambda\}) B\left(\lambda_{1}\right) B\left(\lambda_{2}\right) \ldots \widehat{B\left(\lambda_{k}\right.}\right) \ldots B\left(\lambda_{l}\right) \Omega
\end{gathered}
$$

where

$$
M_{j}(\lambda,\{\lambda\})=g\left(\lambda-\lambda_{j}\right) \prod_{k \neq j}^{l} f\left(\lambda-\lambda_{j}\right) \alpha^{N}\left(\lambda_{j}\right)
$$

we have, for $j=l+1: A(\lambda) B\left(\lambda_{1}\right) B\left(\lambda_{2}\right) \ldots B\left(\lambda_{l}\right) B\left(\lambda_{l+1}\right) \Omega$

$$
\begin{aligned}
& \left.=A(\lambda) B\left(\lambda_{l+1}\right) B\left(\lambda_{1}\right) B\left(\lambda_{2}\right) \ldots B\left(\lambda_{l}\right) \Omega \quad \text { since }[B(\lambda), B(\mu)]=0\right) \\
& =f\left(\lambda-\lambda_{l+1}\right) B\left(\lambda_{l+1}\right) A(\lambda) B\left(\lambda_{1}\right) B\left(\lambda_{2}\right) \ldots B\left(\lambda_{l}\right) \Omega+g\left(\lambda-\lambda_{l+1}\right) B(\lambda) A\left(\lambda_{l+1}\right) B\left(\lambda_{1}\right) B\left(\lambda_{2}\right) \ldots B\left(\lambda_{l}\right) \Omega \\
& =f\left(\lambda-\lambda_{l+1}\right) B\left(\lambda_{l+1}\right)\left[\prod_{j=1}^{l} f\left(\lambda-\lambda_{j}\right) \alpha^{N}(\lambda) B\left(\lambda_{1}\right) B\left(\lambda_{2}\right) \ldots B\left(\lambda_{l}\right) \Omega+\right. \\
& \left.\left.\sum_{j=1}^{l} g\left(\lambda-\lambda_{j}\right) \prod_{k \neq j}^{l} f\left(\lambda_{j}-\lambda_{k}\right) \alpha^{N}\left(\lambda_{j}\right) B\left(\lambda_{1}\right) B\left(\lambda_{2}\right) \ldots \widehat{B\left(\lambda_{j}\right.}\right) \ldots B\left(\lambda_{l}\right) \Omega\right]+ \\
& g\left(\lambda-\lambda_{l+1}\right) B(\lambda)\left[\prod_{j=1}^{l} f\left(\lambda_{l+1}-\lambda_{j}\right) \alpha^{N}\left(\lambda_{l+1}\right) B\left(\lambda_{1}\right) B\left(\lambda_{2}\right) \ldots B\left(\lambda_{l}\right) \Omega+\right. \\
& \left.\left.\sum_{j=1}^{l} g\left(\lambda_{l+1}-\lambda_{j}\right) \prod_{k \neq j}^{l} f\left(\lambda_{j}-\lambda_{k}\right) \alpha^{N}\left(\lambda_{l+1}\right) B\left(\lambda_{1}\right) B\left(\lambda_{2}\right) \ldots \widehat{B\left(\lambda_{j}\right.}\right) \ldots B\left(\lambda_{l}\right) \Omega\right] \\
& =\prod_{j=1}^{l+1} f\left(\lambda-\lambda_{j}\right) \alpha^{N}(\lambda) B\left(\lambda_{1}\right) B\left(\lambda_{2}\right) \ldots B\left(\lambda_{l+1}\right) \Omega+ \\
& g\left(\lambda-\lambda_{l+1}\right) \prod_{j=1}^{l} f\left(\lambda_{l+1}-\lambda_{j}\right) \alpha^{N}\left(\lambda_{l+1}\right) B\left(\lambda_{1}\right) B\left(\lambda_{2}\right) \ldots B\left(\lambda_{l}\right) B(\lambda) \Omega+ \\
& \left.\sum_{j=1}^{l} g\left(\lambda-\lambda_{j}\right) \prod_{k \neq j}^{l+1} f\left(\lambda_{j}-\lambda_{k}\right) \alpha^{N}\left(\lambda_{j}\right) B\left(\lambda_{1}\right) B\left(\lambda_{2}\right) \ldots \widehat{B\left(\lambda_{j}\right.}\right) \ldots B\left(\lambda_{l}\right) B\left(\lambda_{l+1}\right) \Omega+ \\
& g\left(\lambda-\lambda_{l+1}\right) \sum_{j=1}^{l} g\left(\lambda_{l+1}-\lambda_{j}\right) \prod_{k \neq j}^{l} f\left(\lambda_{j}-\lambda_{k}\right) \alpha^{N}\left(\lambda_{l+1}\right) B\left(\lambda_{1}\right) B\left(\lambda_{2}\right) \ldots \widehat{B\left(\lambda_{j}\right) \ldots B\left(\lambda_{l}\right) B(\lambda) \Omega}
\end{aligned}
$$




$$
\begin{array}{r}
\Longrightarrow A(\lambda) B\left(\lambda_{1}\right) B\left(\lambda_{2}\right) \ldots B\left(\lambda_{l+1}\right) \Omega=\prod_{k=1}^{l+1} f\left(\lambda-\lambda_{k}\right) \alpha^{N}(\lambda) B\left(\lambda_{1}\right) B\left(\lambda_{2}\right) \ldots B\left(\lambda_{l+1}\right) \Omega+ \\
\left.\sum_{k=1}^{l+1} M_{k}(\lambda,\{\lambda\}) B\left(\lambda_{1}\right) B\left(\lambda_{2}\right) \ldots \widehat{B\left(\lambda_{k}\right.}\right) \ldots B\left(\lambda_{l+1}\right) \Omega
\end{array}
$$

\section{A.4 Eigenvalue of Bethe vectors when acted on by $S^{3}(3.80)$}

In order to find the eigenvalues of the Bethe vectors when $S^{3}$ operates on them, we first note that we can rewrite the commutator of the Lax operator with the total spin operator.

$$
\left[L_{n}(\lambda), S^{\alpha}\right]=\sum_{k=1}^{N} \frac{1}{2}\left[L_{n}(\lambda), \sigma_{k}^{\alpha}\right]=\frac{1}{2}\left[L_{n}(\lambda), \sigma_{n}^{\alpha}\right]
$$

When $k \neq n$, the commutators go to zero. Hence, only the term with $k=n$ remains. We use the expression of the Lax operator from equation (3.27) along with the fact that the identity operator trivially commutes with $\sigma_{n}^{\alpha}$ to get

$$
\frac{1}{2}\left[L_{n}(\lambda), \sigma_{n}^{\alpha}\right]=\frac{i}{4} \sum_{\beta=1}^{3}\left[\sigma^{\beta} \otimes \sigma_{n}^{\beta}, \sigma_{n}^{\alpha}\right]=-\frac{1}{2} \sum_{\beta, \gamma=1}^{3} \epsilon^{\beta \alpha \gamma} \sigma^{\beta} \otimes \sigma_{n}^{\gamma}
$$

where we used the relation $\sigma_{i} \sigma_{j}=\delta^{i j} I+\epsilon^{i j k} \sigma_{k}$.

From the above, we can also say that $\epsilon^{\beta \alpha \gamma}=i\left[\sigma_{\gamma}, \sigma_{\alpha}\right]$.

$$
\Longrightarrow-\frac{1}{2} \sum_{\beta, \gamma=1}^{3} \epsilon^{\beta \alpha \gamma} \sigma^{\beta} \otimes \sigma_{n}^{\gamma}=-\frac{1}{2} \sum_{\gamma=1}^{3} i\left[\sigma^{c} \otimes \sigma_{n}^{c}, \sigma_{n}^{a}\right]=-\frac{1}{2}\left[L_{n}(\lambda), \sigma^{\alpha}\right]
$$

Hence, we have

$$
\left[L_{n}(\lambda), S^{\alpha}\right]_{q}=-\frac{1}{2}\left[L_{n}(\lambda), \sigma^{\alpha}\right]_{a u x}
$$

where the first commutator is taken in the quantum space while the second is taken in the auxilliary space.

Now, we rewrite the commutator of the monodromy and $S^{\alpha}$. 


$$
\begin{gathered}
{\left[T(\lambda), S^{\alpha}\right]_{q}=\sum_{n=1}^{N} L_{N}(\lambda) \ldots\left[L_{n}(\lambda), S^{\alpha}\right] \ldots L_{1}(\lambda)=-\frac{1}{2} \sum_{n=1}^{N} L_{N}(\lambda) \ldots\left[L_{n}(\lambda), \sigma^{\alpha}\right]_{a u x} \ldots L_{1}(\lambda)} \\
=-\frac{1}{2}\left[T(\lambda), \sigma^{\alpha}\right]_{\text {aux }}
\end{gathered}
$$

Therefore, we have

$$
\left[T_{a}(\lambda), \frac{1}{2} \sigma^{\alpha}+S^{\alpha}\right]=0
$$

Now, for $S^{3}$ in particular,

$$
\begin{gathered}
{\left[S^{3}, T(\lambda)\right]=\frac{1}{2}\left[T(\lambda), \sigma^{3}\right]} \\
\Longrightarrow\left[S^{3}, T\right]=\frac{1}{2}\left[\left(\begin{array}{ll}
A & B \\
C & D
\end{array}\right),\left(\begin{array}{cc}
1 & 0 \\
0 & -1
\end{array}\right)\right]=\frac{1}{2}\left\{\left(\begin{array}{cc}
A & -B \\
C & -D
\end{array}\right)-\left(\begin{array}{cc}
A & B \\
-C & -D
\end{array}\right)\right\} \\
\Longrightarrow\left[S^{3},\left(\begin{array}{cc}
A & B \\
C & D
\end{array}\right)\right]=\left(\begin{array}{cc}
0 & -B \\
C & 0
\end{array}\right) \\
\Longrightarrow\left[S^{3}, B\right]=-B
\end{gathered}
$$

Now, we know that $S^{3}=\frac{1}{2} \sum_{k=1}^{N} \sigma_{k}^{3}$. For the reference state, we have

$$
S^{3} \Omega=\frac{N}{2} \Omega
$$

For a general state $\Phi=B\left(\lambda_{1}\right) B\left(\lambda_{2}\right) \ldots B\left(\lambda_{l}\right) \Omega$, and using the relation derived above, $S^{3} B=$ $B S^{3}-B$, we have the following. 


$$
\begin{gathered}
S^{3} B\left(\lambda_{1}\right) B\left(\lambda_{2}\right) \ldots B\left(\lambda_{l}\right) \Omega=B\left(\lambda_{1}\right) S^{3} B\left(\lambda_{2}\right) \ldots B\left(\lambda_{l}\right) \Omega-B\left(\lambda_{1}\right) B\left(\lambda_{2}\right) \ldots B\left(\lambda_{l}\right) \Omega \\
=B\left(\lambda_{1}\right) B\left(\lambda_{2}\right) S^{3} B\left(\lambda_{3}\right) \ldots B\left(\lambda_{l}\right) \Omega-2\left\{B\left(\lambda_{1}\right) B\left(\lambda_{2}\right) \ldots B\left(\lambda_{l}\right) \Omega\right\} \\
\cdot \\
\cdot \\
\cdot \\
=B\left(\lambda_{2}\right) \ldots B\left(\lambda_{l}\right) S^{3} \Omega-l \Phi \\
=\left(\frac{N}{2}-l\right) \Phi
\end{gathered}
$$

Hence, the eigenvalue of a Bethe vector when acted upon by $S^{3}$ is $\left(\frac{N}{2}-l\right)$. 


\section{A.5 Momentum, Energy, and S-Matrix Element of a type-M Complex (3.104, 3.105, 3.106)}

(i) Momentum of a type-M Complex

We have

$$
\begin{gathered}
e^{i p_{M}}(\lambda)=e^{i \sum_{m} p_{0}(\lambda+i m)} \\
\Longrightarrow i p_{M}(\lambda)=\sum_{m} \log \frac{\lambda+i(m+1 / 2)}{\lambda+i(m-1 / 2)} \\
\Longrightarrow p_{M}(\lambda)=\frac{1}{i} \log \prod_{m} \frac{\lambda+i(m+1 / 2)}{\lambda+i(m-1 / 2)} \\
\Longrightarrow p_{M}(\lambda)=\frac{1}{i} \log \left\{\frac{\lambda+i(M+1 / 2)}{\lambda+i(M-1 / 2)} \frac{\lambda+i(M-1 / 2)}{\lambda+i(M-3 / 2)} \cdots \frac{\lambda+i(-M+1 / 2)}{\lambda+i(-M-1 / 2)}\right\} \\
\Longrightarrow p_{M}(\lambda)=\frac{1}{i} \log \left\{\frac{\lambda+i(M+1 / 2)}{\lambda-i(M+1 / 2)}\right\}
\end{gathered}
$$

(ii) Energy of a type-M Complex

The energy in the ferromagnetic case considered can be found using the relation

$$
\begin{gathered}
\epsilon_{M}(\lambda)=-\frac{1}{2} \frac{d}{d \lambda} p_{M}(\lambda) \\
\epsilon_{M}(\lambda)=-\frac{1}{2 i}\left\{\frac{1}{\lambda+i(M+1 / 2)}-\frac{1}{\lambda-i(M+1 / 2)}\right\} \\
\epsilon_{M}(\lambda)=-\frac{1}{2 i}\left\{\frac{-i M-i / 2-i M-i / 2}{\lambda^{2}+(M+1 / 2)^{2}}\right\} \\
\epsilon_{M}(\lambda)=\frac{1}{2}\left\{\frac{2 M+1}{\lambda^{2}+(M+1 / 2)^{2}}\right\}
\end{gathered}
$$

The energy can be rewritten in terms of $p$ excluding $\lambda$.

$$
\begin{aligned}
e^{i p_{M}}=\frac{\lambda+i(M+1 / 2)}{\lambda-i(M+1 / 2)}= & \frac{\lambda+i(M+1 / 2)}{\lambda-i(M+1 / 2)} \frac{\sqrt{\lambda^{2}+(M+1 / 2)^{2}}}{\sqrt{\lambda^{2}+(M+1 / 2)^{2}}}=e^{2 i \cos ^{-1}\left(\frac{\lambda}{\sqrt{\lambda^{2}+(M+1 / 2)^{2}}}\right)} \\
& \Longrightarrow \cos \left(\frac{p_{M}}{2}\right)=\frac{\lambda}{\sqrt{\lambda^{2}+(M+1 / 2)^{2}}}
\end{aligned}
$$




$$
\begin{gathered}
\cos p_{M}=2 \cos ^{2}\left(\frac{p_{M}}{2}\right)-1=\frac{2 \lambda^{2}}{\lambda^{2}+(M+1 / 2)^{2}}-1 \\
\Longrightarrow 1-\cos p_{M}=\frac{2 \lambda^{2}+2(M+1 / 2)^{2}-2 \lambda^{2}}{\lambda^{2}+(M+1 / 2)^{2}}=\frac{1}{2} \frac{2 M+1}{\lambda^{2}+(M+1 / 2)^{2}} \\
\Longrightarrow \epsilon_{M}(\lambda)=\frac{1}{2 M+1}\left(1-\cos p_{M}\right)
\end{gathered}
$$

(iii) S-Matrix Element of a type-M Complex

The scattering matrix element comes from the function $\phi(\lambda-\mu)$ in equation (3.87).

$$
S(\lambda-\mu)=\frac{\lambda+\mu+i}{\lambda-\mu-i}
$$

The S-matrix element for the scattering of a complex of type 0 with one of type $\mathrm{M}$ is given by

$$
\begin{gathered}
S_{0, M}(\lambda)=\prod_{m=-M}^{m=M} \frac{\lambda_{m}+i}{\lambda_{m}-i}=\prod_{m=-M}^{m=M} \frac{\lambda+i m+i}{\lambda+i m-i} \\
=\frac{\lambda+i(M+1)}{\lambda+i(M-1)} \frac{\lambda+i M}{\lambda+i(M-2)} \ldots \frac{\lambda+i(2-M)}{\lambda-i M)} \frac{\lambda+i(1-M)}{\lambda+i(1+M)} \\
=\frac{\lambda+i(M+1)}{\lambda-i(M+1)} \cdot \frac{\lambda+i M}{\lambda-i M} \cdot \frac{\lambda+i(M-1)}{\lambda-i(M-1)} \cdot \frac{\lambda+i(M-2)}{\lambda-i(M-2)} \cdots \frac{\lambda+i(2-M)}{\lambda-i(2-M)} \cdot \frac{\lambda+i(1-M)}{\lambda-i(1-M)} \\
\Longrightarrow S_{0, M}(\lambda)=\frac{\lambda+i M}{\lambda-i M} \frac{\lambda+i(M+1)}{\lambda-i(M+1)}
\end{gathered}
$$


The scattering matrix element for the scattering of a complex of type $\mathrm{M}$ with one of type $\mathrm{N}$ is given by

$$
\begin{aligned}
& S_{M, N}(\lambda)=\prod_{m, n=-M,-N}^{M, N} \frac{\lambda_{m, n}+i}{\lambda_{m, n}-i}=\prod_{m, n=-M,-N}^{M, N} \frac{\lambda+i(m+n)+i}{\lambda-i(m+n)-i} \\
& =\prod_{n=-N}^{N} \frac{\lambda+i(M+1)+i n}{\lambda+i(M-1)+i n} \cdot \frac{\lambda+i M+i n}{\lambda+i(M-2)+i n} \cdot \frac{\lambda+i(M-1)+i n}{\lambda+i(M-3)+i n} \ldots \frac{\lambda+i(2-M)+i n}{\lambda-i M+i n} \cdot \frac{\lambda+i(1-M)+i n}{\lambda-i(1+M)+i n} \\
& =\prod_{n=-N}^{N} \frac{\lambda+i M+i n}{\lambda-i M+i n} \cdot \frac{\lambda+i(M+1)+i n}{\lambda-i(M+1)+i n} \\
& =\left(\frac{\lambda+i(M+N)}{\lambda-i(M-N)} \cdot \frac{\lambda+i(M+N+1)}{\lambda-i(M-N+1)}\right) \cdot\left(\frac{\lambda+i(M+N-1)}{\lambda-i(M-N+1)} \cdot \frac{\lambda+i(M+N)}{\lambda-i(M-N+2)}\right) \\
& \times\left(\frac{\lambda+i(M+N-2)}{\lambda-i(M-N+2)} \cdot \frac{\lambda+i(M+N-1)}{\lambda-i(M-N+3)}\right) \cdots\left(\frac{\lambda+i(M-N+2)}{\lambda-i(M+N-2)} \cdot \frac{\lambda+i(M-N+3)}{\lambda-i(M+N-1)}\right) \\
& \quad \times\left(\frac{\lambda+i(M-N+1)}{\lambda-i(M+N-1)} \cdot \frac{\lambda+i(M-N+2)}{\lambda-i(M+N)}\right) \cdot\left(\frac{\lambda+i(M-N)}{\lambda-i(M+N)} \cdot \frac{\lambda+i(M-N+1)}{\lambda-i(M+N+1)}\right) \\
& =\prod_{L=|M-N|}^{M+N} \frac{\lambda+i L}{\lambda-i L} \cdot \frac{\lambda+i(L+1)}{\lambda-i(L+1)} \prod_{L=|M-N|}^{M+N} S_{0, L}(\lambda)
\end{aligned}
$$




\section{A.6 Results used in the Derivation of the Dimensionality of the Spin Chain (3.2.6)}

(i) $Z(N, l, q)=\frac{N-2 l+1}{N-l+1}\left(\begin{array}{c}N-l+1 \\ q\end{array}\right)\left(\begin{array}{l}l-1 \\ q-1\end{array}\right)$

The relation can be proved by induction, as was done by Bethe in his original paper. To prove this, we assume that the relation is true for $Z(N-2 q, l-q, q-\nu)$.

$$
\begin{gathered}
\Longrightarrow Z(N, l, q)=\sum_{\nu=0}^{q-1}\left(\begin{array}{c}
(N-2 q)+\nu \\
\nu
\end{array}\right) \frac{(N-2 q)-2(l-q)+1}{(N-2 q)-(l-q)+1}\left(\begin{array}{c}
(N-2 q)-(l-q)+1 \\
q-\nu
\end{array}\right)\left(\begin{array}{c}
(l-q)-1 \\
q-\nu-1
\end{array}\right) \\
\Longrightarrow Z(N, l, q)=\sum_{\nu=0}^{q-1}\left(\begin{array}{c}
N-2 q+\nu \\
\nu
\end{array}\right) \frac{N-2 l+1}{N-l-q+1}\left(\begin{array}{c}
N-l-q+1 \\
q-\nu
\end{array}\right)\left(\begin{array}{c}
l-q-1 \\
q-\nu-1
\end{array}\right)
\end{gathered}
$$

We use the relation $\sum_{r=0}^{k}\left(\begin{array}{c}n-m \\ r\end{array}\right)\left(\begin{array}{c}m \\ k-r\end{array}\right)=\left(\begin{array}{l}n \\ k\end{array}\right)$ to have

$$
\begin{gathered}
\left(\begin{array}{c}
N-2 q+\nu \\
\nu
\end{array}\right)=\sum_{s=0}^{\nu}\left(\begin{array}{c}
N-2 q+\nu+1-l \\
\nu-s
\end{array}\right)\left(\begin{array}{c}
l-1 \\
s
\end{array}\right) \\
\Longrightarrow Z(N, l, q)=\sum_{\nu=0}^{q-1} \sum_{s=0}^{\nu}\left(\begin{array}{c}
N-2 q+\nu+1-l \\
\nu-s
\end{array}\right)\left(\begin{array}{c}
l-1 \\
s
\end{array}\right) \frac{N-2 l+1}{N-l-q+1}\left(\begin{array}{c}
N-l-q+1 \\
q-\nu
\end{array}\right)\left(\begin{array}{c}
l-q-1 \\
q-\nu-1
\end{array}\right)
\end{gathered}
$$

Multiplying and dividing by $(q-s)$ !, we get

$$
\begin{array}{r}
\Longrightarrow Z(N, l, q)=\sum_{\nu=0}^{q-1} \sum_{s=0}^{\nu} \frac{(N-2 q+\nu+1-l) !}{(\nu-s) !(N-l-2 q+s+1) !} \frac{(N-l-q+1) !}{(q-\nu) !(N-l-2 q+\nu+1) !} \\
\times \frac{N-2 l+1}{N-l-q+1}\left(\begin{array}{c}
l-q-1 \\
q-\nu-1
\end{array}\right)\left(\begin{array}{c}
l-1 \\
s
\end{array}\right) \times \frac{(q-s) !}{(q-s) !}(
\end{array}
$$




$$
\begin{aligned}
\Longrightarrow Z(N, l, q)=\frac{N-2 l+1}{N-l-q+1} \sum_{s=0}^{q-1}\left(\begin{array}{c}
l-1 \\
s
\end{array}\right)\left(\begin{array}{r}
N-l-q+1 \\
q-s
\end{array}\right) \\
\times \sum_{\nu=s}^{q-1}\left(\begin{array}{c}
q-s \\
\nu-s
\end{array}\right)\left(\begin{array}{l}
l-q-1 \\
q-\nu-1
\end{array}\right)
\end{aligned}
$$

using the same relation as before.

$$
Z(N, l, q) \quad=\quad \frac{N-2 l+1}{N-l-q+1} \sum_{s=0}^{q-1}\left(\begin{array}{c}
l-1 \\
s
\end{array}\right)\left(\begin{array}{c}
N-l-q+1 \\
q-s
\end{array}\right)\left(\begin{array}{c}
l-s-1 \\
q-s-1
\end{array}\right)
$$

Multiplying and dividing by $(q-1)$ !, we get

$$
\begin{gathered}
\Longrightarrow Z(N, l, q)=\frac{N-2 l+1}{N-l-q+1} \sum_{s=0}^{q-1} \frac{(l-1) !}{s !(l-s-1) !} \frac{(l-s-1) !}{(q-s-1) !(l-q) !} \frac{(q-1) !}{(q-1) !} \\
\times\left(\begin{array}{c}
N-l-q+1 \\
q-s
\end{array}\right) \\
\Longrightarrow Z(N, l, q)=\frac{N-2 l+1}{N-l-q+1}\left(\begin{array}{c}
l-1 \\
q-1
\end{array}\right) \sum_{s=0}^{q-1}\left(\begin{array}{c}
q-1 \\
s
\end{array}\right)\left(\begin{array}{c}
N-l-q+1 \\
q-s
\end{array}\right)
\end{gathered}
$$

Using the above combination relation once more,

$$
\begin{gathered}
Z(N, l, q)=\frac{N-2 l+1}{N-l-q+1}\left(\begin{array}{c}
l-1 \\
q-1
\end{array}\right) \sum_{s=0}^{q-1}\left(\begin{array}{c}
q-1 \\
s
\end{array}\right)\left(\begin{array}{c}
N-l-q+1 \\
q-s
\end{array}\right) \\
\Longrightarrow Z(N, l, q)=\frac{N-2 l+1}{N-l-q+1}\left(\begin{array}{c}
l-1 \\
q-1
\end{array}\right)\left(\begin{array}{c}
N-l \\
q
\end{array}\right)=\frac{N-2 l+1}{N-l+1}\left(\begin{array}{c}
l-1 \\
q-1
\end{array}\right)\left(\begin{array}{c}
N-l+1 \\
q
\end{array}\right)
\end{gathered}
$$

The relation is true for $l=1, q=1$, since $Z(N, 1,1)=N-1$.

For $l=2, q=1$, we have $Z(N, 2,1)=\frac{N-3}{N-1}\left(\begin{array}{l}1 \\ 0\end{array}\right)\left(\begin{array}{c}N-1 \\ 1\end{array}\right)=N-3$, which agrees with what should be expected. 
Hence, the relation holds for all values of $l$ and $q$, by induction.

(ii) $\sum_{l}(N-2 l+1)\left(\left(\begin{array}{c}N \\ l\end{array}\right)-\left(\begin{array}{c}N \\ l-1\end{array}\right)\right)=2^{N}$

$$
\begin{gathered}
\sum_{l=0}^{N / 2}(N-2 l+1)\left(\left(\begin{array}{c}
N \\
l
\end{array}\right)-\left(\begin{array}{c}
N \\
l-1
\end{array}\right)\right) \\
=(N+1)\left(2^{N-1}+\frac{1}{2}\left(\begin{array}{c}
N \\
N / 2
\end{array}\right)\right)-(N+1)\left(2^{N-1}-\frac{1}{2}\left(\begin{array}{c}
N \\
N / 2
\end{array}\right)\right)-2 \sum_{l=0}^{N / 2} l\left(\left(\begin{array}{c}
N \\
l
\end{array}\right)-\left(\begin{array}{c}
N \\
l-1
\end{array}\right)\right) \\
=(N+1)\left(\begin{array}{c}
N \\
N / 2
\end{array}\right)-2 \sum_{l=0}^{N / 2} l\left(\begin{array}{c}
N \\
l
\end{array}\right)+2 \sum_{l=1}^{N / 2} l\left(\begin{array}{c}
N \\
l-1
\end{array}\right) \\
=(N+1)\left(\begin{array}{c}
N \\
N / 2
\end{array}\right)-2 \sum_{l=0}^{N / 2} l\left(\begin{array}{c}
N \\
l
\end{array}\right)+2 \sum_{l=0}^{N / 2-1}\left(\begin{array}{c}
l+1 \\
l
\end{array}\right)\left(\begin{array}{c}
N \\
l
\end{array}\right) \\
=(N+1)\left(\begin{array}{c}
N \\
N / 2
\end{array}\right)-N\left(\begin{array}{c}
N \\
N / 2
\end{array}\right)+2 \sum_{l=0}^{N / 2-1}\left(\begin{array}{c}
N \\
l
\end{array}\right) \\
=(N+1)\left(\begin{array}{c}
N \\
N / 2
\end{array}\right)-N\left(\begin{array}{c}
N \\
N / 2
\end{array}\right)+2\left(2^{N-1}-\frac{1}{2}\left(\begin{array}{c}
N \\
N / 2
\end{array}\right)\right)=2^{N}
\end{gathered}
$$




\section{A.7 Results Used in Analysis of Antiferromagnetic Ground State in Thermodynamic Limit (3.2.7)}

(i) Density function of Antiferromagnetic Ground State

We define

$$
\begin{gathered}
\theta_{n}(\lambda)=2 \tan ^{-1}\left(\frac{2 \lambda}{n}\right) \\
a_{n}(\lambda)=\frac{1}{2 \pi} \frac{d \theta_{n}}{d \lambda}
\end{gathered}
$$

From equation (3.153), we have

$$
\begin{gathered}
\frac{2}{1+4 \lambda^{2}}=\pi \rho_{0}(\lambda)+\int_{-\infty}^{\infty} \frac{\rho_{0}(\mu)}{1+(\lambda-\mu)^{2}} d \mu \\
\Longrightarrow \frac{d}{d \lambda}\left(2 \tan ^{-1}(2 \lambda)\right)=2 \pi \rho_{0}(\lambda)+\int_{-\infty}^{\infty} \rho_{0}(\mu) \frac{d}{d \lambda}\left(2 \tan ^{-1}(\lambda-\mu)\right) d \mu \\
\Longrightarrow 2 \pi a_{1}(\lambda)=2 \pi \rho_{0}(\lambda)+2 \pi \int_{-\infty}^{\infty} a_{2}(\lambda-\mu) \rho_{0}(\mu) d \mu \\
\Longrightarrow a_{1}(\lambda)=\rho_{0}(\lambda)+\left(a_{2} * \rho_{0}\right)(\lambda) \\
\Longrightarrow a_{1}(\omega)=\rho_{0}(\omega)+a_{2}(\omega) \rho_{0}(\omega)
\end{gathered}
$$

We first need to find the form of $a_{n}(\omega)$.

$$
a_{n}(\omega)=\int_{-\infty}^{\infty} d \lambda a_{n}(\lambda) e^{i \omega \lambda}=\int_{-\infty}^{\infty} \frac{d \lambda}{2 \pi} \frac{2 \cdot \frac{2}{n}}{1+\frac{4 \lambda^{2}}{n^{2}}} e^{i \omega \lambda}=\int_{-\infty}^{\infty} \frac{d \lambda}{2 \pi} \frac{n}{n^{2}+4} e^{i \omega \lambda}
$$

Converting this into a contour integral, we get

$$
\oint \frac{d z}{2 \pi} \frac{n}{z^{2}+4} e^{i \omega z}
$$

which has poles at $z= \pm \frac{i}{2}$ Taking a semicircular contour in the upper half-plane, we have

$$
\left.\operatorname{Res} f(z)\right|_{z=i n / 2}=\lim _{z \rightarrow i n / 2}\left(z-\frac{i n}{2}\right) f(z)=\lim _{z \rightarrow i n / 2}\left(z-\frac{i n}{2}\right) \frac{n e^{i \omega z}}{2 \pi\left(z-\frac{i n}{2}\right)\left(z+\frac{i n}{2}\right)}=\frac{e^{-\frac{n \omega}{2}}}{2 \pi i}
$$


Because of the residue theorem, we have

$$
\oint \frac{d z}{2 \pi} \frac{n}{z^{2}+4} e^{i \omega z}=2 \pi i \cdot \frac{e^{-\frac{n \omega}{2}}}{2 \pi i}=e^{-\frac{n \omega}{2}}=\int_{\text {straight }} f(z) d z+\int_{\text {arc }} f(z) d z
$$

We require that $\frac{n \omega}{2}$ is negative for the integral over the arc to vanish.

$$
\Longrightarrow a_{n}(\omega)=e^{-n|\omega| / 2}
$$

Substituting in equation (A.60), we obtain

$$
\begin{gathered}
a_{1}(\omega)=\rho_{0}(\omega)+a_{2}(\omega) \rho_{0}(\omega)=\rho_{0}(\omega)\left(1+a_{2}(\omega)\right) \\
\Longrightarrow e^{-|\omega|}=\rho_{0}(\omega)+a_{2}(\omega) \rho_{0}(\omega)=\rho_{0}(\omega)\left(1+e^{-|\omega|}\right) \\
\Longrightarrow \rho_{0}(\omega)=\frac{1}{2 \cosh (\omega / 2)}
\end{gathered}
$$

We now need to do the reverse FT.

$$
\rho_{0}(\lambda)=\int_{-\infty}^{\infty} \frac{d \omega}{2 \pi} \rho_{0}(\omega) e^{-i \omega \lambda}=\frac{1}{2 \cosh (\pi \lambda)}
$$

(ii) Energy of Antiferromagnetic Ground State

$$
\begin{gathered}
E=N \int_{-\infty}^{\infty} \epsilon_{0}(\lambda) \rho_{0}(\lambda) d \lambda=-N \pi \int_{-\infty}^{\infty} a_{1}(\lambda) \rho_{0}(\lambda) d \lambda \\
=-N \pi \int_{-\infty}^{\infty} a_{1}(\omega) \rho_{0}(\omega) \frac{d \omega}{2 \pi}=-\frac{N}{2} \int_{-\infty}^{\infty} \frac{e^{-|\omega| / 2}}{e^{|\omega| / 2}+e^{-|\omega| / 2}} d \omega=-\frac{N}{2} \int_{-\infty}^{\infty} \frac{e^{-|\omega|}}{1+e^{-|\omega|}} d \omega \\
=-N \int_{0}^{\infty} d \omega \frac{e^{-\omega}}{1+e^{-\omega}}=\int_{0}^{\infty} d \omega \frac{d\left(\log \left(1+e^{-\omega}\right)\right)}{d \omega} \\
=-N \log 2
\end{gathered}
$$


(iii) Verification of expression for $\sigma(\lambda)$ used in AFM triplet state

It needs to be verified that the solution

$$
\sigma(\lambda)=\frac{1}{2 \pi} \int_{-\infty}^{\infty} \hat{\sigma}(\xi) e^{-i \lambda \xi} d \xi ; \hat{\sigma}(\xi)=-\frac{1}{1+e^{-|\xi|}}
$$

satisfies the equation

$$
\sigma(\lambda)+\frac{1}{\pi} \int_{-\infty}^{\infty} \frac{\sigma(\mu))}{1+(\lambda-\mu)^{2}} d \mu+\delta(\lambda)=0
$$

$$
\begin{aligned}
\frac{1}{\pi} \int_{-\infty}^{\infty} \frac{\sigma(\mu)}{1+(\lambda-\mu)^{2}} d \mu & =\frac{1}{2 \pi^{2}} \int_{-\infty}^{\infty} d \mu \int_{-\infty}^{\infty} d \xi \frac{1}{1+(\lambda-\mu)^{2}} \frac{e^{-i \mu \xi}}{1+e^{-|\xi|}} \\
& =\frac{1}{2 \pi^{2}} \int_{-\infty}^{\infty} d \xi \frac{e^{-i \lambda \xi}}{1+e^{-|\xi|}} \int_{-\infty}^{\infty} d(\mu-\lambda) \frac{e^{-i(\mu-\lambda) \xi}}{1+(\mu-\lambda)^{2}}
\end{aligned}
$$

$$
\oint d z \frac{e^{-i z \xi}}{(z+i)(z-i)}=2 \pi i \lim _{y \rightarrow-i}\left(\frac{e^{-i z \xi}}{z-i}\right)=2 \pi i\left(\frac{e^{-\xi}}{-2 i}\right)=-\pi e^{-\xi}
$$

$$
\begin{gathered}
\Longrightarrow \frac{1}{\pi} \int_{-\infty}^{\infty} \frac{\sigma(\mu)}{1+(\lambda-\mu)^{2}} d \mu=-\frac{1}{2 \pi^{2}} \int_{-\infty}^{\infty} d \xi \frac{e^{-i \lambda \xi} \pi e^{-\xi}}{1+e^{-|\xi|}}=-\frac{1}{2 \pi} \int_{-\infty}^{\infty} d \xi \frac{e^{-i(\lambda+1) \xi}}{1+e^{-|\xi|}} \\
=-\frac{1}{2 \pi} \int_{-\infty}^{\infty} d \xi \frac{e^{-i \lambda \xi}}{1+e^{-|\xi|}}-\frac{1}{2 \pi} \int_{-\infty}^{\infty} d \xi \frac{\left(e^{-\xi}+1\right) e^{-i \lambda \xi}}{1+e^{-|\xi|}} \\
=-\sigma(\lambda)-\frac{1}{2 \pi} \int_{-\infty}^{\infty} e^{-i \lambda \xi} d \xi \\
=-\sigma(\lambda)-\delta(\lambda)
\end{gathered}
$$

$$
\Longrightarrow \sigma(\lambda)+\frac{1}{\pi} \int_{-\infty}^{\infty} \frac{\sigma(\mu))}{1+(\lambda-\mu)^{2}} d \mu+\delta(\lambda)=0
$$

(iv) Energy of Antiferromagnetic Triplet State 


$$
\begin{gathered}
\epsilon(\lambda)=\int_{-\infty}^{\infty} h(\mu) \sigma(\lambda-\mu) d \mu=\frac{1}{4 \pi} \int_{-\infty}^{\infty} d \mu \int_{-\infty}^{\infty} d \xi \frac{1}{\mu^{2}+1 / 4} \cdot \frac{e^{-i(\lambda-\mu) \xi}}{1+e^{-|\xi|}} \\
=\frac{1}{4 \pi} \int_{-\infty}^{\infty} d \xi \frac{e^{-i \lambda \xi}}{1+e^{-|\xi|}} \int_{-\infty}^{\infty} d \mu \frac{e^{i \mu \xi}}{\mu^{2}+1 / 4}=\frac{1}{4 \pi} \int_{-\infty}^{\infty} d \xi \frac{e^{-i \lambda \xi}}{1+e^{-|\xi|}}\left[2 \pi i\left(\frac{e^{-|\xi| / 2}}{i}\right)\right] \\
=\frac{1}{2} \int_{-\infty}^{\infty} d \xi \frac{e^{-|\xi| / 2}}{1+e^{-|\xi|}} e^{-i \lambda \xi}=\frac{\pi}{2 \cosh (\pi \lambda)}
\end{gathered}
$$

In the last line, the result from the derivation in the ground state was used.

(v) Momentum of Antiferromagnetic Triplet State

$$
k(\lambda)=\int_{-\infty}^{\infty} p(\mu) \sigma(\lambda-\mu) d \mu=\frac{1}{2 \pi i} \int_{-\infty}^{\infty} d \mu \int_{-\infty}^{\infty} d \xi \ln \left(\frac{\mu+1 / 2}{\mu-1 / 2}\right) \cdot \frac{e^{-i(\lambda-\mu) \xi}}{1+e^{-|\xi|}}
$$

The Fourier transform of the momentum is $p(\xi)=2 \pi \frac{e^{-|\xi|}}{i \xi}$. Defining $\gamma=\mu-\lambda$, we can write the above integral as

$$
\begin{array}{r}
k(\lambda)=\int_{-\infty}^{\infty} d \xi \frac{e^{-|\xi|}}{1+e^{-|\xi|}} \frac{e^{i \xi \gamma}}{i \xi}=\int_{-\infty}^{\infty} d \xi \rho_{0}(\xi)\left(\int^{\lambda} d \gamma e^{i \xi \gamma}\right)=2 \pi \int^{\lambda} d \gamma \rho_{0}(\gamma) \\
=\int^{\lambda} d \gamma \frac{\pi}{\cosh (\pi \gamma)}=\tan ^{-1}(\sinh (\pi \lambda))
\end{array}
$$

(vi) Verification of expression for $\omega(\lambda)$

It needs to be verified that

$$
\omega(\lambda)=-\frac{2}{\pi\left(1+4 \lambda^{2}\right)}
$$

is a solution to

$$
\pi \omega(\lambda)+\Phi_{0,1 / 2}^{\prime}(\lambda)=-\int_{-\infty}^{\infty} \frac{\omega(\mu)}{1+(\lambda-\mu)^{2}} d \mu
$$

We start from the left hand side. 


$$
\begin{aligned}
\pi \omega(\lambda)+\Phi_{0,1 / 2}^{\prime}(\lambda)= & \left(-\frac{2}{\pi\left(1+4 \lambda^{2}\right)}\right)+\frac{d}{d \lambda}\left[\tan ^{-1}(2 \lambda)+\tan ^{-1}(2 \lambda / 3)\right] \\
& =-\frac{2}{1+4 \lambda^{2}}+\frac{2}{1+4 \lambda^{2}}+\frac{2 / 3}{1+4 \lambda^{2} / 9}=\frac{6}{4 \lambda^{2}+9}
\end{aligned}
$$

Now, we turn our attention to the right hand side of the equation.

$$
\begin{gathered}
-\int_{-\infty}^{\infty} \frac{\omega(\mu)}{1+(\lambda-\mu)^{2}} d \mu=\frac{2}{\pi} \int_{-\infty}^{\infty} d \mu \frac{1}{\left(1+(\lambda-\mu)^{2}\right)\left(1+4 \mu^{2}\right)} \\
=\frac{2}{\pi} \cdot \frac{1}{16 \lambda^{4}+40 \lambda^{2}+9} \int_{-\infty}^{\infty} d \mu\left\{\frac{16 \lambda^{2}+12+(8 \mu)(4 \lambda)}{1+4 \mu^{2}}+\frac{4 \lambda^{2}+(4 \lambda)(2 \lambda)-3-(4 \lambda)(2 \mu)}{1+(\lambda-\mu)^{2}}\right\} \\
\left.=\frac{2}{\pi} \cdot \frac{1}{16 \lambda^{4}+40 \lambda^{2}+9}\left[4 \lambda\left(\log \left(4 \mu^{2}+1\right)\right)-\log \left((\lambda-\mu)^{2}+1\right)\right)+\left(3-4 \lambda^{2}\right) \tan ^{-1}(\lambda-\mu)+\left(8 \lambda^{2}+6\right) \tan ^{-1}(2 \mu)\right]_{\mu=-\infty}^{\infty} \\
=\frac{2}{\pi} \cdot \frac{1}{\left(4 \lambda^{2}+1\right)\left(4 \lambda^{2}+9\right)}\left[\left(3-4 \lambda^{2}\right)(-\pi)+\left(8 \lambda^{2}+6\right)(\pi)\right] \\
=\frac{2}{\pi} \cdot \frac{1}{\left(4 \lambda^{2}+1\right)\left(4 \lambda^{2}+9\right)}\left(3 \pi\left(4 \lambda^{2}+1\right)\right)=\frac{6}{4 \lambda^{2}+9}
\end{gathered}
$$

Therefore, both sides are clearly equal and the solution holds. 
(vii) Energy contribution of $\omega(\lambda)$

The contribution of $\omega(\lambda)$ to the total energy is the following.

$$
\begin{aligned}
\int_{-\infty}^{\infty} h(\lambda) \omega\left(\lambda-\lambda_{s}\right) d \lambda= & -\frac{1}{4 \pi} \int_{-\infty}^{\infty} d \lambda \int_{-\infty}^{\infty} d \xi e^{-|\xi| / 2-i\left(\lambda-\lambda_{s}\right) \xi} \cdot \frac{1}{\lambda^{2}+\frac{1}{4}} \\
& =-\frac{1}{4 \pi} \int_{-\infty}^{\infty} d \xi e^{-|\xi| / 2+i \lambda_{s} \xi} \int_{-\infty}^{\infty} d \lambda \frac{e^{-i \lambda \xi}}{\lambda^{2}+\frac{1}{4}} \\
& =-\frac{1}{4 \pi} \int_{-\infty}^{\infty} d \xi e^{-|\xi| / 2+i \lambda_{s} \xi}\left[2 \pi i \cdot \frac{e^{-|\xi| / 2}}{-i}\right] \\
& =\frac{1}{2} \int_{\infty}^{\infty} d \xi e^{-|\xi|+i \lambda_{s} \xi}=\frac{1}{\lambda_{s}^{2}+1}=-h_{1 / 2}\left(\lambda_{s}\right)
\end{aligned}
$$

(viii) Momentum contribution of $\omega(\lambda)$

The contribution of $\omega(\lambda)$ to the total energy is the following.

$$
\begin{gathered}
\int_{-\infty}^{\infty} p(\lambda) \omega\left(\lambda-\lambda_{s}\right) d \lambda=-\frac{1}{2 \pi} \int_{-\infty}^{\infty} d \lambda \int_{-\infty}^{\infty} d \xi e^{-|\xi| / 2-i\left(\lambda-\lambda_{s}\right) \xi}\left(2 \pi \frac{e^{-|\xi|}}{i \xi}\right)=-\int_{-\infty}^{\infty} d \xi e^{-3|\xi| / 2} \cdot \frac{e^{i \gamma \xi}}{i \xi} \\
=-\int_{-\infty}^{\infty} d \xi e^{-3|\xi| / 2}\left(\int^{\lambda} d \gamma e^{i \xi \gamma}\right)=-\int^{\lambda} d \gamma \frac{2}{\gamma^{2}+1}=-2 \tan ^{-1}(\lambda)=-p_{1 / 2}(\lambda)
\end{gathered}
$$




\section{Bibliography}

[1] Faddeev, L. D. "How Algebraic Bethe Ansatz works for integrable model." arXiv preprint hep-th/9605187 (1996).

[2] Takhtadzhyan, L.A., and Faddeev, L.D. "Spectrum and scattering of excitations in the one-dimensional isotropic Heisenberg model." Zapiski Nauchnykh Seminarov POMI 109 (1981): $134-178$.

[3] Bethe, Hans. "On the theory of metals, I. Eigenvalues and eigenfunctions of a linear chain of atoms." In Selected Works Of Hans A Bethe: (With Commentary), pp. 155-183. 1997.

[4] Sutherland, Bill. Beautiful Models: 70 Years of Exactly Solved Quantum Many-body Problems. New Jersey: World Scientific, 2004.

[5] de Leeuw, Marius and Constantin Candu. "Spin Chains." Lecture, Introduction to Integrability, Zurich, 2013.

[6] Beisert, Niklas. "Lecture Notes." Lecture, Integrability in QFT and AdS/CFT, Zurich, 2014.

[7] Doikou, Anastasia. "Selected Topics in Classical Integrability." Int.J.Mod.Phys. A27 1230003 arXiv:1110.4235 (2012) 